\title{
Measurements of the Higgs boson width and anomalous $H V V$ couplings from on-shell and off-shell production in the four-lepton final state
}

\author{
A. M. Sirunyan et al. \\ (CMS Collaboration)
}

(Received 1 January 2019; published 11 June 2019)

\begin{abstract}
Studies of on-shell and off-shell Higgs boson production in the four-lepton final state are presented, using data from the CMS experiment at the LHC that correspond to an integrated luminosity of $80.2 \mathrm{fb}^{-1}$ at a center-of-mass energy of $13 \mathrm{TeV}$. Joint constraints are set on the Higgs boson total width and parameters that express its anomalous couplings to two electroweak vector bosons. These results are combined with those obtained from the data collected at center-of-mass energies of 7 and $8 \mathrm{TeV}$, corresponding to integrated luminosities of 5.1 and $19.7 \mathrm{fb}^{-1}$, respectively. Kinematic information from the decay particles and the associated jets are combined using matrix element techniques to identify the production mechanism and to increase sensitivity to the Higgs boson couplings in both production and decay. The constraints on anomalous $H V V$ couplings are found to be consistent with the standard model expectation in both the onshell and off-shell regions. Under the assumption of a coupling structure similar to that in the standard model, the Higgs boson width is constrained to be $3.2_{-2.2}^{+2.8} \mathrm{MeV}$ while the expected constraint based on simulation is $4.1_{-4.0}^{+5.0} \mathrm{MeV}$. The constraints on the width remain similar with the inclusion of the tested anomalous $H V V$ interactions.
\end{abstract}

DOI: $10.1103 /$ PhysRevD.99.112003

\section{INTRODUCTION}

The standard model (SM) of particle physics postulates the existence of a Higgs field responsible for the generation of the masses of fundamental particles. The excitation of this field is known as the Higgs boson $(H)$ [1-7]. The observation of an $H$ boson with a mass of around $125 \mathrm{GeV}$ by the ATLAS and CMS Collaborations [8-10] is consistent with the expectations of the SM, but further tests of the properties of this particle, such as its width and the structure of its couplings to the known SM particles, are needed to determine its nature.

The CMS and ATLAS experiments have set constraints of $\Gamma_{H}<13 \mathrm{MeV}$ at $95 \%$ confidence level (C.L.) on the $H$ boson total width [11-15] using the off-shell production method [16-18], which relies on the relative measurement of off-shell and on-shell production. The upper bound on $\Gamma_{H}$ was set considering the gluon fusion and electroweak (EW) production mechanisms in the analysis. The precision on $\Gamma_{H}$ from on-shell measurements of the width of the resonance peak alone is approximately $1 \mathrm{GeV}$ [19-21], which is significantly worse than the result from the

*Full author list given at the end of the article.

Published by the American Physical Society under the terms of the Creative Commons Attribution 4.0 International license. Further distribution of this work must maintain attribution to the author(s) and the published article's title, journal citation, and DOI. Funded by SCOAP . off-shell method. The constraint on the $H$ boson lifetime is equivalent to a lower bound on the width and was derived from the flight distance in the CMS detector as $\Gamma_{H}>$ $3.5 \times 10^{-9} \mathrm{MeV}$ at $95 \%$ C.L. [13]. The SM expectation of the width of the $H$ boson is around $4 \mathrm{MeV}$ [22].

The CMS [13,23-27] and ATLAS [28-33] experiments have set constraints on the spin-parity properties and anomalous couplings of the $H$ boson, finding its quantum numbers to be consistent with $J^{P C}=0^{++}$, but allowing small anomalous couplings to two EW gauge bosons (anomalous $H V V$ couplings). Off-shell signal production may be enhanced in the presence of these anomalous $H V V$ couplings $[11,13,22,34-36]$. As a result, the measurement of $\Gamma_{H}$ using the off-shell technique may be affected by these deviations of the $H$ boson couplings from the SM expectations. An attempt to measure $\Gamma_{H}$ using the off-shell technique while including anomalous $H V V$ interactions has been made by the CMS experiment [13]. In that previous study, constraints are placed on $\Gamma_{H}$ and the on-shell crosssection fraction $f_{\Lambda Q}$ that expresses an anomalous coupling contribution sensitive to the invariant mass of the $H$ boson, using a realistic treatment of interference between the $H$ boson signal and the continuum background. Extending the application of the off-shell technique to a wider range of anomalous $H V V$ contributions, studied previously using onshell $H$ boson production [27], is the goal of this paper.

The presented investigation on the $H$ boson width targets both gluon fusion and EW production mechanisms and tests the effects of possible anomalous $H V V$ couplings in 
either production or decay. Nevertheless, it still relies on the knowledge of coupling ratios between the off-shell and onshell production, the dominance of the top quark loop in the gluon fusion production mechanism, and the absence of new particle contributions in the loop. A violation of the last assumption by itself would be a manifestation of physics beyond the SM (BSM), which may become evident if the measured width deviates from the SM expectation. The measured width may also deviate from the SM expectation if the $H$ boson has new BSM decay channels or the known channels have non-SM rates. Therefore, the measurement of the width complements the search for $H$ boson decay to invisible or undetected particles, and the measurement of the $H$ boson couplings to the known SM particles.

The data sample used in this analysis corresponds to integrated luminosities of $35.9 \mathrm{fb}^{-1}$ collected in 2016 and $41.5 \mathrm{fb}^{-1}$ collected in 2017 during Run 2 of the CERN LHC at a center-of-mass energy of $13 \mathrm{TeV}$. These results are combined with results obtained earlier from the data collected at center-of-mass energies of $7 \mathrm{TeV}$ (in 2011), $8 \mathrm{TeV}$ (in 2012), and $13 \mathrm{TeV}$ (in 2015), corresponding to integrated luminosities of $5.1,19.7$, and $2.7 \mathrm{fb}^{-1}$, respectively [25,27]. The increase in either energy and integrated luminosity leads to substantial improvement in the precision of the width measurement using the off-shell technique, either under the assumption of SM couplings or with BSM effects.

This analysis follows closely the general $H \rightarrow 4 \ell$ (leptons $\ell=e$ or $\mu$ ) selection and reconstruction documented in Ref. [21] using the data collected in 2016, and the on-shell study of anomalous $H V V$ couplings with the combined 2015 and 2016 data set in Ref. [27]. Many of the technical details of the search for a scalar resonance $X \rightarrow$ $Z Z$ at high mass in Run 2 data, documented in Ref. [37], are also shared in the analyses presented here. The rest of the paper is organized as follows. The phenomenology of anomalous $H V V$ interactions is discussed in Sec. II. The CMS detector, reconstruction techniques, and Monte Carlo (MC) simulation methods are introduced in Sec. III. The addition of the 2017 data to that used in Refs. [21,27], and the relevant differences in the detector and reconstruction techniques are also discussed in this section. The details of the analysis are discussed in Secs. IV and V, and the results are presented in Sec. VI. We provide a summary of these results in Sec. VII.

\section{PHENOMENOLOGY OF ANOMALOUS HVV INTERACTIONS}

The constraints on $\Gamma_{H}$ are set using the off-shell production method, which considers the $H$ boson production relationship between the on-shell $\left(105<m_{4 \ell}<\right.$ $140 \mathrm{GeV})$ and off-shell $\left(m_{4 \ell}>220 \mathrm{GeV}\right)$ regions. Denoting each production mechanism with $v v \rightarrow H \rightarrow$ $V V \rightarrow 4 \ell$ for the $H$ boson coupling to either strong $(v v=g g)$ or EW $(v v=W W, Z Z, Z \gamma, \gamma \gamma)$ vector bosons in its production, the on-shell and off-shell $H$ boson signal yields are related by [16]

$$
\sigma_{v v \rightarrow H \rightarrow 4 \ell}^{\text {on-shell }} \propto \mu_{v v H} \quad \text { and } \quad \sigma_{v v \rightarrow H \rightarrow 4 \ell}^{\text {off-shell }} \propto \mu_{v v H} \Gamma_{H},
$$

where $\mu_{v v H}$ is defined as the on-shell signal strength, the ratio of the observed number of on-shell four-lepton events relative to the SM expectation. This ratio is interpreted as either $\mu_{F}$ for $H$ boson production via gluon fusion $(g g H)$ or in association with a $t \bar{t}(t \bar{t} H)$ or $b \bar{b}$ pair $(b \bar{b} H)$, or $\mu_{V}$ for $H$ boson production via vector boson fusion (VBF) or in association with an EW vector boson $W$ or $Z(V H)$. There is sizable interference between the $H$ boson signal and the continuum background in the off-shell region [17], contrary to on-shell production, and this formalism scales the interference contribution with $\sqrt{\mu_{v v H} \Gamma_{H}}$.

This analysis is based on a phenomenological framework [22,38-59] that describes the anomalous couplings of a Higgs-like boson to two gauge bosons, such as $W W, Z Z$, $Z \gamma, \gamma \gamma$, and $g g$. These couplings appear in either the production of the $H$ boson or its decay, regardless of the $m_{4 \ell}$ region in which the $H$ boson is produced. The relationship in Eq. (1) is therefore meant to imply concurrent variations in $v v H$ couplings in both on-shell and off-shell regions. The coupling of the $H$ boson to two gluons is assumed to be as in the SM, via quark loops with Yukawa couplings to quarks, where the contribution from the top-quark is dominant. This assumption is valid as long as the production is dominated by the top-quark loop and no new particles contribute to this loop. The Yukawa couplings also appear in direct interactions with fermionantifermion pairs, such as in $t \bar{t} H$ and $b \bar{b} H$ productions. These interactions are of less importance in this study, since they are highly suppressed at high off-shell mass, but they are included in the analysis of the on-shell $H$ boson production with similar assumptions as in the case of production via gluon fusion. Variation of the $H V V$ couplings, in either the VBF or $V H$ productions, or the $H \rightarrow$ $4 \ell$ decay, are allowed to depend on anomalous coupling contributions.

In the following, we assume that the $H$ boson couples to two gauge bosons $\mathrm{VV}$, such as $W W, Z Z, Z \gamma$ or $\gamma \gamma$, which in turn couple to fermions, either four leptons in $H$ boson decay, or quarks or leptons in its production or in the decay of associated EW bosons. It is assumed that the $H$ boson does not couple to fermions through a new heavy state, generating a so-called contact interaction [57,58]. However, the inclusion of amplitude terms pertaining to contact interactions is equivalent to the anomalous $H V V$ couplings already tested [25] under the assumption of flavor universality in $V f \bar{f}$ couplings. Both approaches test three general tensor structures allowed by Lorentz symmetry, with form factors $F_{i}\left(q_{1}^{2}, q_{2}^{2}\right)$ in front of each term, where $q_{1}$ and $q_{2}$ are the four-momenta of the two difermion states, such as $\left(e^{+} e^{-}\right)$and $\left(\mu^{+} \mu^{-}\right)$in the $H \rightarrow e^{+} e^{-} \mu^{+} \mu^{-}$ 
decay, and equivalent states in production. We also fix all lepton and quark couplings to vector bosons according to SM expectations. Relaxing this requirement would make it equivalent to flavor nonuniversal couplings of the contact terms, but would also introduce too many unconstrained parameters, which cannot be tested with the present data sample. Only the lowest order operators, or lowest order terms in the $\left(q_{j}^{2} / \Lambda^{2}\right)$ form-factor expansion, are tested, where $\Lambda$ is the energy scale of new physics.

The signal scattering amplitude describing the interaction between a spin-zero $H$ boson and two spin-one gauge bosons $V V$ is written as [54]

$$
\begin{aligned}
A \sim & {\left[a_{1}^{V V}-\frac{\kappa_{1}^{V V} q_{1}^{2}+\kappa_{2}^{V V} q_{2}^{2}}{\left(\Lambda_{1}^{V V}\right)^{2}}-\frac{\kappa_{3}^{V V}\left(q_{1}+q_{2}\right)^{2}}{\left(\Lambda_{Q}^{V V}\right)^{2}}\right] m_{V 1}^{2} \epsilon_{V 1}^{*} \epsilon_{V 2}^{*} } \\
& +a_{2}^{V V} f_{\mu \nu}^{*(1)} f^{*(2) \mu \nu}+a_{3}^{V V} f_{\mu \nu}^{*(1)} \tilde{f}^{*(2) \mu \nu}
\end{aligned}
$$

In this expression of the scattering amplitude, $\epsilon_{i}$ is the polarization vector of gauge boson $V_{i}, f^{(i) \mu \nu}=\epsilon_{i}^{\mu} q_{i}^{\nu}-\epsilon_{i}^{\nu} q_{i}^{\mu}$ is a scalar tensor constructed from this polarization vector and the momentum of the gauge boson, and $\tilde{f}_{\mu \nu}^{(i)}=$ $\frac{1}{2} \epsilon_{\mu \nu \rho \sigma} f^{(i) \rho \sigma}$ is the pseudoscalar tensor counterpart. When at least one of the gauge bosons $V$ is massive, $m_{V 1}$ is the pole mass of that gauge boson. The scales of BSM physics are denoted with $\Lambda_{1}$ and $\Lambda_{Q}$, so $a_{i}{ }^{V V}$, or $1 / \Lambda_{1}$ and $1 / \Lambda_{Q}$, become the coupling-strength modifiers of the relevant $H V V$ amplitudes, where $a_{i}{ }^{V V}$ may in general be any complex number, and $\left|\kappa_{1,2,3}^{V V}\right|=0$ or 1 are complex numbers. Under the assumption that the couplings are constant and real, the above formulation is equivalent to an effective Lagrangian notation. Therefore, in this paper, the real coupling constants are tested. The above approach allows a sufficiently general test of the $H \rightarrow 4 \ell$ kinematics in decay and equivalent kinematics in production, as discussed below, including production and decay of virtual intermediate photons. If deviations from the SM are detected, a more detailed study of $F_{i}\left(q_{1}^{2}, q_{2}^{2}\right)$ could be performed, eventually providing a measurement of the double-differential cross section for each tensor structure tested.

In the above, the only leading tree-level contributions are $a_{1}^{Z Z} \neq 0$ and $a_{1}^{W W} \neq 0$, and in the following we assume the custodial symmetry $a_{1}^{Z Z}=a_{1}^{W W}$. The rest of the couplings are considered anomalous contributions, which are either tiny contributions arising in the SM due to loop effects or new BSM contributions. The SM loop contributions are not accessible experimentally with the available data. Among anomalous contributions, considerations of symmetry and gauge invariance require $\kappa_{1}^{Z Z}=\kappa_{2}^{Z Z}=-\exp \left(i \phi_{\Lambda 1}^{Z Z}\right), \kappa_{1}^{\gamma \gamma}=$ $\kappa_{2}^{\gamma \gamma}=0, \kappa_{1}^{g g}=\kappa_{2}^{g g}=0, \kappa_{1}^{Z \gamma}=0$, and $\kappa_{2}^{Z \gamma}=-\exp \left(i \phi_{\Lambda 1}^{Z \gamma}\right)$. While not strictly required, the same symmetry is considered in the $W W$ case $\kappa_{1}^{W W}=\kappa_{2}^{W W}=-\exp \left(i \phi_{\Lambda 1}^{W W}\right)$.
Neither $H Z \gamma$ nor $H \gamma \gamma$ couplings produce a sizable offshell enhancement, since there is no interplay between the vector bosons or the $H$ boson going off-shell, and there is no off-shell threshold for these couplings. Therefore, offshell treatment for these couplings can be ignored. While the $a_{2,3}^{Z \gamma}$ and $a_{2,3}^{\gamma \gamma}$ terms are tested in the Run 1 analysis [25], the precision of those constraints is still not competitive with the on-shell photon measurements in $H \rightarrow Z \gamma$ and $\gamma \gamma$. Therefore, we omit those measurements in this paper. The $\Lambda_{1}^{Z_{\gamma}}$ coupling, on the other hand, can only be observed with off-shell photons decaying to a pair of fermions, so it is considered in the on-shell analysis. The $\Lambda_{Q}$ term depends only on the invariant mass of the $H$ boson, so its contribution is not distinguishable from the SM in the on-shell region and is only testable through the off-shell region. Tight constraints are already set on this parameter in the Run 1 analysis [13], so it is also not considered in this paper.

In the following, the $Z Z$ labels for the $Z Z$ interactions are omitted, and we use a generic notation $a_{i}$ to denote $a_{3}, a_{2}$, $1 / \Lambda_{1}$, and $1 / \Lambda_{1}^{Z \gamma}$, which are the four couplings tested in this paper as listed in Table I. Furthermore, the $W W$ measurements are integrated into the $Z Z$ measurements assuming $a_{i}^{Z Z}=a_{i}^{W W}$. The $H W W$ contributions appear in the $\mathrm{VBF}$ and $W H$ productions. This assumption does not affect the kinematic analysis of events because there is very little difference in kinematic distributions in events initiated by either $W W$ or $Z Z$ fusion. However, this assumption may affect the interpretation of the results should a different relationship between $a_{i}^{Z Z}$ and $a_{i}^{W W}$ be assumed. Therefore, such a scenario is discussed in more detail below by introducing the parameter $r_{a i}$, following Ref. [25], as

$$
r_{a i}=\frac{a_{i}^{W W} / a_{1}^{W W}}{a_{i} / a_{1}} .
$$

Including the parameter $r_{a i}$ in the probability parametrization despite the lack of sensitivity of the data would introduce complexity without a comparable gain in physics content. We proceed with the analysis assuming $r_{a i}=1$,

TABLE I. List of the anomalous $H V V$ couplings considered in the measurements assuming a spin-zero $H$ boson. The definition of the effective fractions $f_{a i}$ is discussed in the text and the translation constants are the cross-section ratios corresponding to the processes $H \rightarrow 2 e 2 \mu$ with the $H$ boson mass $m_{H}=125 \mathrm{GeV}$ and calculated using JHUGEN [47,50,54].

\begin{tabular}{lccc}
\hline \hline $\begin{array}{l}\text { Anomalous } \\
\text { coupling }\end{array}$ & $\begin{array}{c}\text { Coupling } \\
\text { phase }\end{array}$ & $\begin{array}{c}\text { Effective } \\
\text { fraction }\end{array}$ & Translation constant \\
\hline$a_{3}$ & $\phi_{a 3}$ & $f_{a 3}$ & $\sigma_{1} / \sigma_{3}=6.53$ \\
$a_{2}$ & $\phi_{a 2}$ & $f_{a 2}$ & $\sigma_{1} / \sigma_{2}=2.77$ \\
$\Lambda_{1}$ & $\phi_{\Lambda 1}$ & $f_{\Lambda 1}$ & $\sigma_{1} / \tilde{\sigma}_{\Lambda 1}=1.47 \times 10^{4} \mathrm{TeV}^{-4}$ \\
$\Lambda_{1}^{Z \gamma}$ & $\phi_{\Lambda 1}^{Z \gamma}$ & $f_{\Lambda 1}^{Z \gamma}$ & $\sigma_{1} / \tilde{\sigma}_{\Lambda 1}^{Z \gamma}=5.80 \times 10^{3} \mathrm{TeV}^{-4}$ \\
\hline \hline
\end{tabular}


but point out below how results could be reinterpreted should a different value be assumed.

Most systematic uncertainties cancel when taking ratios to the total cross section, so measurements of $a_{i}$ relative to the dominant SM-like contribution $a_{1}$ are the preferred approach. For this purpose, the effective fractional $Z Z$ cross sections $f_{a i}$ and phases $\phi_{a i}$ are defined as

$$
\begin{aligned}
f_{a i} & =\frac{\left|a_{i}\right|^{2} \sigma_{i}}{\sum_{j=1,2,3 \ldots}\left|a_{j}\right|^{2} \sigma_{j}}, \\
\phi_{a i} & =\arg \left(\frac{a_{i}}{a_{1}}\right),
\end{aligned}
$$

where $\sigma_{i}$ is the cross section for the process corresponding to $a_{i}=1, a_{j \neq i}=0$, while $\tilde{\sigma}_{\Lambda 1}$ is the effective cross section for the process corresponding to $\Lambda_{1}=1 \mathrm{TeV}$, given in units of $\mathrm{fb} \mathrm{TeV}^{4}$. The cross-section ratios are quoted in Table I. The $a_{i} / a_{1}$ ratios can be obtained from the ratio $f_{a i} / f_{a 1}$, the cross-section ratios, and the phase $\phi_{a i}$ as

$$
\frac{a_{i}}{a_{1}}=\sqrt{\frac{f_{a i}}{f_{a 1}} \frac{\sigma_{1}}{\sigma_{i}}} e^{i \phi_{a i}} .
$$

The effective fractions $f_{a i}$ are bounded between 0 and 1 and do not depend on the coupling convention. In most cases, uncertainties on these measurements scale with integrated luminosity as $1 / \sqrt{\mathcal{L}}$ until effects of interference become important. Furthermore, the values of $f_{a i}$ have a simple interpretation as the fractional size of the BSM contribution for the $H \rightarrow 2 e 2 \mu$ decay. For example, $f_{a i}=0$ indicates a pure SM-like $H$ boson, $f_{a i}=1$ gives a pure BSM particle, and $f_{a i}=0.5$ means that the two couplings contribute equally to the $H \rightarrow 2 e 2 \mu$ process.

As mentioned above in application to Eq. (3), the measurement of $f_{a i}$ is performed under the $r_{a i}=1$ assumption. Let us denote this to be an effective $f_{a i}^{\text {eff }}$. Without such an assumption, there is a certain dependence of $f_{a i}$ on $r_{a i}$ and $f_{a i}^{\mathrm{eff}}$, such that $f_{a i}=f_{a i}^{\mathrm{eff}}$ for $r_{a i}=1$. This dependence is different for different processes, such as VBF production or $H \rightarrow 4 \ell$ decay, where the latter case is in fact independent of $r_{a i}$ because the $H W W$ coupling does not affect this decay process. In the former case, let us consider the relative contributions of $W W$ and $Z Z$ fusion on-shell. For example, the ratio of $\mathrm{VBF}$ cross sections driven by $W W$ and $Z Z$ fusion is $\sigma_{1}^{W W} / \sigma_{1}^{Z Z}=2.59$ for the SM tree-level couplings under custodial symmetry $a_{1}^{W W}=$ $a_{1}^{Z Z}$ at $13 \mathrm{TeV} p p$ collision energy. The same ratio for the $C P$-odd couplings is $\sigma_{3}^{W W} / \sigma_{3}^{Z Z}=3.15$, where $\sigma_{3}^{V V}$ are calculated for $a_{3}^{W W}=a_{3}^{Z Z}$. The dependence of $f_{a i}$ on $r_{a i}$ and $f_{a i}^{\text {eff }}$, as measured in the VBF process, becomes

$f_{a i}=\left[1+\left(1 / f_{a i}^{\mathrm{eff}}-1\right)\left(\sigma_{i}^{Z Z}+r_{a i}^{2} \sigma_{i}^{W W}\right) /\left(\sigma_{i}^{Z Z}+\sigma_{i}^{W W}\right)\right]^{-1}$, where custodial symmetry $a_{1}^{W W}=a_{1}^{Z Z}$ is assumed and the effects of interference between $W W$ and $Z Z$ fusion are negligible and are therefore ignored.

All of the above discussion, including Eq. (2), describes the production of a resonance via gluon fusion, VBF with associated jets, or associated production with an EW vector boson, $V H$. These mechanisms, along with the $t \bar{t} H$ and $b \bar{b} H$ production, are considered in the analysis of the spinzero hypothesis of the $H$ boson, where the gluon fusion production is expected to dominate. It is possible to study $H V V$ interactions using the kinematics of particles produced in association with the $H$ boson, such as VBF jets or vector boson daughters in $V H$ production, as we show below. More details can be found in, e.g., Ref. [54] and the experimental application in Refs. [26,27]. While the $q_{i}^{2}$ range in the $H V V$ process does not exceed approximately $100 \mathrm{GeV}$ because of the kinematic bound, no such bound exists in the associated production, so consideration of more restricted $q_{i}^{2}$ ranges might be required [54]. However, we only consider that the $q_{i}^{2}$ range is not restricted in the allowed phase space.

\section{THE CMS DETECTOR, SIMULATION, AND RECONSTRUCTION}

The $H \rightarrow 4 \ell$ decay candidates are reconstructed in the CMS detector [60]. The CMS detector is comprised of a silicon pixel and strip tracker, a lead tungstate crystal electromagnetic calorimeter (ECAL), and a brass/scintillator hadron calorimeter, each composed of a barrel and two end cap sections, all within a superconducting solenoid of $6 \mathrm{~m}$ internal diameter, providing a magnetic field of $3.8 \mathrm{~T}$. Extensive forward calorimetry complements the coverage provided by the barrel and end cap detectors. Outside the solenoid are the gas-ionization detectors for muon measurements, which are embedded in the steel flux-return yoke. A detailed description of the CMS detector, together with a definition of the coordinate system used and the relevant kinematic variables, can be found in Ref. [60].

The JHUGEN 7.0.2 [47,50,54,59] Monte Carlo (MC) program is used to simulate anomalous couplings in the $H$ boson production and $H \rightarrow Z Z / Z \gamma^{*} / \gamma^{*} \gamma^{*} \rightarrow 4 \ell$ decay. The gluon fusion production is simulated with the POWHEG 2 [61-65] event generator at next-to-leading order (NLO) in QCD, and simulation with the MINLO [66] program at NLO in QCD is used for evaluation of systematic uncertainties related to modeling of two associated jets. The kinematics of events produced in gluon fusion with two associated jets are also modified by anomalous $\mathrm{Hg} g$ couplings. These effects are studied using JHUGEN, and it is found that the kinematic distributions relevant for this analysis are not affected significantly.

The production of the $H$ boson through VBF, in association with a $W$ or $Z$ boson, or with a $t \bar{t}$ pair, is simulated using both JHUGEN at LO in QCD and POWHEG 
at NLO in QCD. Production in association with a $b \bar{b}$ pair is simulated only at LO in QCD via JHUGEN. In the VBF, $V H$, and $t \bar{t} H$ production modes, the JHUGEN and POWHEG simulations are explicitly compared after parton showering in the SM case, and no significant differences are found in kinematic observables. Therefore, the JHUGEN simulation is adopted to describe kinematics in the VBF, $V H$, and $t \bar{t} H$ production modes with anomalous couplings in the onshell region, with expected yields taken from the POWHEG simulation. The POWHEG program is used to simulate wide resonances at masses ranging from $115 \mathrm{GeV}$ to $3 \mathrm{TeV}$, produced in gluon fusion, VBF, or $V H$. The events from the POWHEG simulation are later reweighted using the package for the matrix element likelihood approach (MELA) [9,47,50,54,59] to model off-shell $H$ boson production distributions, as discussed below.

The $g g \rightarrow Z Z / Z \gamma^{*} \rightarrow 4 \ell$ background process is simulated with MCFM 7.0.1 [18,67-69]. The vector boson scattering and triple-gauge-boson $(V V V)$ backgrounds are obtained by reweighting the POWHEG simulation with the matrix elements provided by the MELA package using the MCFM and JHUGEN matrix elements, and the reweighted simulation is checked against the predictions of the PHANTOM 1.3 [70] simulation. Both the MCFM and PHANTOM generators allow one to model the $H$ boson signal, background, and their interference in the off-shell production. However, they do not allow modeling of the anomalous interactions considered in this analysis. Therefore, a combined program has been developed for both gluon fusion and VBF with triple-gauge-boson production based on the modeling of signal and background scattering amplitudes from MCFM and anomalous contributions in the signal scattering amplitude from JHUGEN. This program is included within the JHUGEN and MELA packages, as detailed in Ref. [22]. A large number of MC events with anomalous couplings in the signal and their interference with background have been generated with these packages. The simulated events also include alternative weights to model various anomalous couplings in the signal.

In the gluon fusion process, the factorization and renormalization scales are chosen to be running as $m_{4 \ell} / 2$. In order to include higher-order QCD corrections, LO, NLO, and next-to-NLO (NNLO) signal cross-section calculations are performed using the MCFM and HNNLO 2 programs [71-73] for a wide range of masses using a narrow width approximation. The ratios between the NNLO and LO values (NNLO K factors) are used to reweight [22] the $m_{4 \ell}$ distributions from the MCFM and JHUGEN simulation at LO in QCD, and a uniform factor of 1.10 across all of the $m_{4 \ell}$ range is applied to normalize the cross section of the $H$ boson production via gluon fusion to the predictions for $m_{4 \ell} \approx 125 \mathrm{GeV}$ at next-to-NNLO $\left(N^{3} \mathrm{LO}\right)$ in QCD [22]. The simulated $m_{4 \ell}$ shapes or yields obtained from the POWHEG simulation of the gluon fusion process are corrected based on the above reweighted distributions. While the
NNLO K factor calculation is directly applicable to the signal contribution, it is approximate for the background and its interference with the signal. The NLO calculation with some approximations [74-77] is available for the background and interference. Comparison with this calculation shows that while there is some increase of the NLO K factor for the interference close to the ZZ threshold, the NLO K factors for the background and interference are consistent with the signal within approximately $10 \%$ in the mass range $m_{4 \ell}>220 \mathrm{GeV}$ relevant for this analysis. We therefore multiply the background and interference contributions by the same NNLO K factor and uniform $N^{3} \mathrm{LO}$ correction, both calculated for signal and including associated uncertainties, and introduce an additional unit factor with a $10 \%$ uncertainty for the background and the square root of this factor for the interference.

The MELA package contains a library of matrix elements from JHUGEN and MCFM for the signal, and MCFM for the background, and is used to apply weights to events in any MC sample to model any other set of anomalous or SM couplings in either on-shell or off-shell production. This matrix element library also allows reweighting of the signal POWHEG simulation of the wide resonances at NLO in QCD in either gluon fusion, VBF, or triple-gauge-boson production to model the signal, background, or their interference.

The main background in this analysis, $q \bar{q} \rightarrow Z Z / Z \gamma^{*} \rightarrow$ $4 \ell$, is estimated from simulation with POWHEG. A fully differential cross section has been computed at NNLO in QCD [78], but it is not yet available in a partonic level event generator. Therefore the NNLO/NLO QCD correction is applied as a function of $m_{4 \ell}$. Additional NLO EW corrections are also applied to this background process in the region $m_{4 \ell}>2 m_{Z}[79,80]$. The parton distribution functions (PDFs) used in this paper belong to the NNPDF 3.0 PDF sets [81]. All MC samples are interfaced to PYTHIA 8 [82] for parton showering, using version 8.212 for the simulation of the 2016 data period and 8.230 for the simulation of the 2017 data period. Simulated events include the contribution from additional $p p$ interactions within the same or adjacent bunch crossings (pileup), and are weighted to reproduce the observed pileup distribution. The MC samples are further processed through a dedicated simulation of the CMS detector based on GEANT4 [83].

The selection of $4 \ell$ events and associated particles closely follows the methods used in the analyses of the Run 1 [24] and Run 2 [21] data sets. The main triggers for the Run 2 analysis select either a pair of electrons or muons, or an electron and a muon. The minimal transverse momentum of the leading electron (muon) is 23 (17) GeV, while that of the subleading lepton is 12 (8) GeV. To maximize the signal acceptance, triggers requiring three leptons with lower $p_{\mathrm{T}}$ thresholds and no isolation requirement are also used, as are isolated single-electron and single-muon triggers with thresholds of 27 and $22 \mathrm{GeV}$ in 2016 , or 35 and $27 \mathrm{GeV}$ in 2017, respectively. The overall trigger efficiency for 
simulated signal events that pass the full selection chain of this analysis is larger than $99 \%$. The trigger efficiency is measured in data using a sample of $4 \ell$ events collected by the single-lepton triggers and is found to be consistent with the expectation from simulation.

Event reconstruction is based on the particle-flow (PF) algorithm [84], which exploits information from all the CMS subdetectors to identify and reconstruct individual particles in the event. The PF candidates are classified as charged hadrons, neutral hadrons, photons, electrons, or muons, and they are then used to build higher-level objects such as jets and lepton isolation quantities. Electrons (muons) are reconstructed within the geometrical acceptance defined by a requirement on the pseudorapidity $|\eta|<2.5(2.4)$ for transverse momentum $p_{T}>7(5) \mathrm{GeV}$ with an algorithm that combines information from the ECAL (muon system) and the tracker. A dedicated algorithm is used to collect the final-state radiation (FSR) of leptons [21].

The reconstructed vertex with the largest value of summed physics-object $p_{\mathrm{T}}^{2}$ is taken to be the primary $p p$ interaction vertex. The physics objects are the jets and the associated missing transverse momentum, taken as the negative vector sum of the $p_{\mathrm{T}}$ of those jets. The jets are clustered using the anti- $k_{\mathrm{T}}$ jet finding algorithm $[85,86]$ with a distance parameter of 0.4 and the associated tracks assigned to the vertex as inputs. Jets must satisfy $p_{\mathrm{T}}>30 \mathrm{GeV}$ and $|\eta|<4.7$ and must be separated from all selected lepton candidates and any selected FSR photons with a requirement on the distance parameter $\Delta R(\ell / \gamma$, jet $)>0.4$, where $(\Delta R)^{2}=(\Delta \phi)^{2}+$ $(\Delta \eta)^{2}$. For event categorization, jets are tagged as $b$-jets using the Combined Secondary Vertex algorithm [87,88], which combines information about impact parameter significance, the secondary vertex, and jet kinematics.

Each lepton track is required to have the ratio of the impact parameter in three dimensions, which is computed with respect to the chosen primary vertex position, and its uncertainty to be less than 4. To discriminate between leptons from prompt $Z$ boson decays and those arising from hadron decays within jets, an isolation requirement for leptons is imposed in the analysis of the 2016 data [21]. For electrons, the isolation variable is included as part of the multivariate training inputs for electron identification in 2017.

We consider three mutually exclusive channels: $H \rightarrow 4 e$, $4 \mu$, and $2 e 2 \mu$. At least two leptons are required to have $p_{T}>10 \mathrm{GeV}$, and at least one is required to have $p_{T}>20 \mathrm{GeV}$. All four pairs of oppositely charged leptons that can be built with the four leptons are required to satisfy $m_{\ell^{+} \ell^{\prime-}}>4 \mathrm{GeV}$ regardless of lepton flavor. The $Z$ candidates are required to satisfy the condition $12<m_{\ell^{+} \ell^{-}}<$ $120 \mathrm{GeV}$, where the invariant mass of at least one of the $Z$ candidates must be larger than $40 \mathrm{GeV}$. The region between 105 and $140 \mathrm{GeV}$ in the four-lepton invariant mass $m_{4 \ell}$ is identified as the on-shell region, and the region above $220 \mathrm{GeV}$ is identified as the off-shell region.
Different sources of leptons such as the decays of heavy flavor jets or light mesons may produce additional background to the $H$ boson signal in any of these decay channels, or the on-shell and off-shell regions. We denote this background collectively as the $Z+X$ background, and employ a data-driven method for its estimation and $m_{4 \ell}$ dependence. The lepton misidentification rates are first derived using $Z+1 \ell$ control regions with relaxed selection requirements on the third lepton, and the extracted rates are then applied on $Z+2 \ell$ control regions, where the two additional leptons with relaxed selection requirements have the same lepton flavor but may have opposite charge [21,24].

\section{ANALYSIS TECHNIQUES AND CATEGORIZATION OF EVENTS}

The full kinematic information from each event using either the $H$ boson decay or associated particles in its production is extracted using discriminants from matrix element calculations. These discriminants use a complete set of mass and angular input observables $\boldsymbol{\Omega}[47,54,59]$ to describe kinematics at LO in QCD. The $p_{T}$ of either the combined $H$ boson and two-jet system for the production discriminant (e.g., $\mathcal{D}^{\mathrm{VBF} / V H}$ ), or the $H$ boson itself for the decay discriminants (e.g., $\mathcal{D}^{\text {dec }}$ ), or for their combination (e.g., $\mathcal{D}^{\mathrm{VBF} / V H+\text { dec }}$ ) is not included in the input observables. This information is not used in the analysis of the $H$ boson width and anomalous couplings, as the $p_{T}$ of the overall system is sensitive to QCD, parton shower, and underlying event uncertainties.

The kinematic discriminants used in this study are computed using the same MELA package that is utilized in simulation. The signal includes both the fourlepton decay kinematics in the processes $H \rightarrow Z Z / Z \gamma^{*} /$ $\gamma^{*} \gamma^{*} \rightarrow 4 \ell$, and kinematics of associated particles in production $H+$ jet, $H+2$ jets, VBF, $W H, Z H, t \bar{t} H$, $t q H$, or $b \bar{b} H$. The background includes $g g$ or $q \bar{q} \rightarrow$ $Z Z / Z \gamma^{*} / \gamma^{*} \gamma^{*} / Z \rightarrow 4 \ell$ processes, and VBF or associated production with a $V$ boson of the $Z Z$ system. Analytical algorithms are available for the cross-checks of the fourlepton kinematics in $H$ decay and $V H$ associated production within the MELA framework and were adopted in the previous CMS analyses [9,10,23].

Kinematic distributions of particles produced in the $H$ boson decay or in association with $H$ boson production are sensitive to the quantum numbers and anomalous couplings of the $H$ boson. In the $1 \rightarrow 4$ process of the $H \rightarrow 4 f$ decay, six observables fully characterize kinematics of the decay products $\boldsymbol{\Omega}^{\text {decay }}=\left\{\theta_{1}, \theta_{2}, \Phi, m_{1}, m_{2}, m_{4 f}\right\}$, while two other angles relate orientation of the decay frame with respect to the production axis, $\boldsymbol{\Omega}^{\text {prod }}=\left\{\theta^{*}, \Phi_{1}\right\}$, as described in Ref. [47]. Moreover, two sets of observables, $\boldsymbol{\Omega}^{\text {assoc, } \mathrm{VBF}}=\left\{\theta_{1}^{\mathrm{VBF}}, \theta_{2}^{\mathrm{VBF}}, \Phi^{\mathrm{VBF}}, q_{1}^{2, \mathrm{VBF}}, q_{2}^{2, \mathrm{VBF}}\right\}$ for the VBF process and $\boldsymbol{\Omega}^{\text {assoc, } V H}=\left\{\theta_{1}^{V H}, \theta_{2}^{V H}, \Phi^{V H}, q_{1}^{2, V H}\right.$, $\left.q_{2}^{2, V H}\right\}$ for the $V H$ process, can also be defined in a similar 

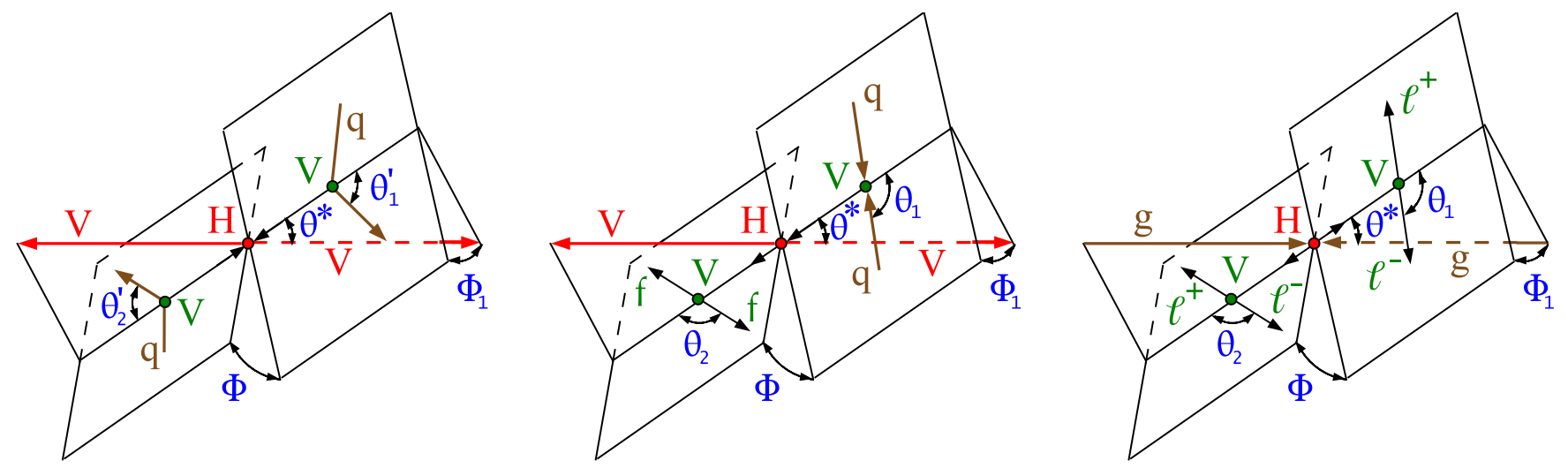

FIG. 1. Three topologies of the $H$ boson production and decay: vector boson fusion $q q \rightarrow V V(q q) \rightarrow H(q q) \rightarrow V V(q q)($ left $)$; associated production $q q \rightarrow V \rightarrow V H \rightarrow(f \bar{f}) H \rightarrow(f \bar{f}) V V$ (middle); and gluon fusion $g g \rightarrow H \rightarrow V V \rightarrow 4 \ell$ (right) representing the topology without associated particles. The incoming particles are shown in brown, the intermediate vector bosons and their fermion daughters are shown in green, the $H$ boson and its vector boson daughters are shown in red, and angles are shown in blue. In the first two cases the production and decay $H \rightarrow V V$ are followed by the same four-lepton decay shown in the third case. The angles are defined in either the $H$ or $V$ boson rest frames [47,54].

way to $\Omega^{\text {decay }}$ for $H$ boson associated production [54]. As a result, 13 kinematic observables, illustrated in Fig. 1, are defined for the $2 \rightarrow 6$ associated production process with subsequent $H$ boson decay to a four-fermion final state.

With up to 13 observables, $\boldsymbol{\Omega}$, sensitive to the $H$ boson anomalous couplings in Eq. (2), it is a challenging task to perform an optimal analysis in a multidimensional space of observables. The MELA approach introduced earlier is designed to reduce the number of observables to the minimum while retaining all essential information. Two types of discriminants were defined for either the production or decay process, and we also combine them into a joint discriminant for the full $2 \rightarrow 6$ process where relevant.

These types of discriminants are

$$
\mathcal{D}_{\text {alt }}(\mathbf{\Omega})=\frac{\mathcal{P}_{\text {sig }}(\boldsymbol{\Omega})}{\mathcal{P}_{\text {sig }}(\mathbf{\Omega})+\mathcal{P}_{\text {alt }}(\mathbf{\Omega})}
$$

and

$$
\mathcal{D}_{\text {int }}(\mathbf{\Omega})=\frac{\mathcal{P}_{\text {int }}(\mathbf{\Omega})}{2 \sqrt{\mathcal{P}_{\text {sig }}(\mathbf{\Omega}) \mathcal{P}_{\text {alt }}(\mathbf{\Omega})}}
$$

where the probability of a certain process $\mathcal{P}$ is calculated using the full kinematics characterized by $\boldsymbol{\Omega}$ for the processes denoted as "sig" for a signal model and "alt" for an alternative model, which could be an alternative $H$ boson production mechanism (used to categorize events), background (to isolate signal), or an alternative $H$ boson coupling model (to measure coupling parameters). The "int" label represents the interference between the two model contributions. The probabilities $\mathcal{P}$ are calculated from the matrix elements provided by the MELA package and are normalized to give the same integrated cross sections in the relevant phase space of each process. Such normalization leads to a balanced distribution of events in the range between 0 and 1 of the $\mathcal{D}_{\text {alt }}$ discriminants, and between
-1 and 1 of $\mathcal{D}_{\text {int }}$. One can apply the Neyman-Pearson lemma to prove that the two discriminants in Eqs. (7) and (8) become the minimal and complete set of optimal observables for the purpose of separating the two processes "sig" and "alt" while including their interference as well [54,59].

The selected events are split into three categories: VBFtagged, $V H$-tagged, and untagged. A set of discriminants $\mathcal{D}_{2 \text { jet }}$ is constructed, following Eq. (7), where $\mathcal{P}_{\text {sig }}$ corresponds to the signal probability for the VBF ( $W H$ or $Z H$ ) production hypothesis in the VBF-tagged ( $\mathrm{VH}$-tagged) category, and $\mathcal{P}_{\text {alt }}$ corresponds to that of $H$ boson production in association with two jets via gluon fusion. When more than two jets pass the selection criteria, the two jets with the highest $p_{T}$ are chosen for the matrix element calculations. Thereby, the $\mathcal{D}_{2 \text { jet }}$ discriminants separate the target production mode of each category from gluon fusion production, in all cases using only the kinematics of the $H$ boson and two associated jets. Figure 2 illustrates these discriminants, designed for the VBF or $\mathrm{VH}$ signal enhancement in the $a_{3}$ coupling analysis for a pseudoscalar contribution. A selection based on the $\mathcal{D}_{\text {bkg }}$ observable, which utilizes information from the $4 \ell$ decay kinematics and invariant mass, and which is discussed in more detail below, is applied in order to enhance the contribution of the signal over the background.

The three on-shell and off-shell categories are summarized in Tables II and III, and their sequential selection criteria are as follows:

(i) VBF-tagged requires exactly four leptons, either two or three jets of which at most one is $b$-quark flavortagged, or more if none are $b$-tagged jets, and $\mathcal{D}_{2 \text { jet }}^{\mathrm{VBF}}>0.5$ using either the $\mathrm{SM}$ or BSM signal hypothesis for the VBF production.

(ii) VH-tagged requires exactly four leptons, either two or three jets, or more if none are $b$-tagged jets, and 

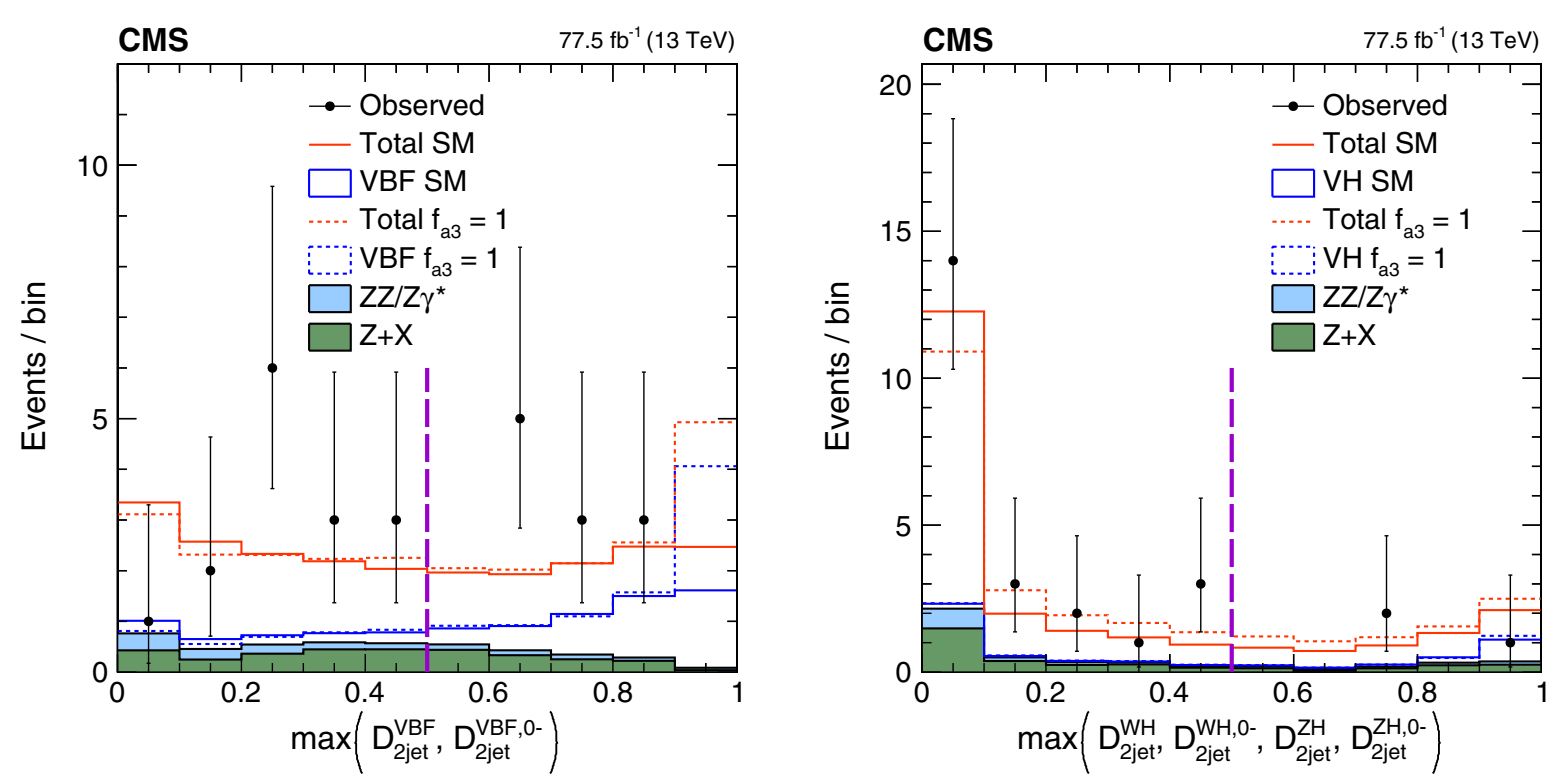

FIG. 2. The distributions of events for $\max \left(\mathcal{D}_{2 \text { jet }}^{\mathrm{VBF}}, \mathcal{D}_{2 \text { jet }}^{\mathrm{VBF}, 0-}\right)($ left $)$ and $\max \left(\mathcal{D}_{2 \text { jet }}^{W H}, \mathcal{D}_{2 \text { jet }}^{W H, 0-}, \mathcal{D}_{2 \text { jet }}^{Z H}, \mathcal{D}_{2 \text { jet }}^{Z H, 0-}\right)$ (right) in the on-shell region in the data from 2016 and 2017 from the analysis of the $a_{3}$ coupling for a pseudoscalar contribution. The requirement $\mathcal{D}_{\mathrm{bkg}}>0.5$ is applied in order to enhance the signal contribution over the background. The VBF signal under both the SM and pseudoscalar hypotheses is enhanced in the region above 0.5 for the former variable, and the $W H$ and $Z H$ signals are similarly enhanced in the region above 0.5 for the latter variable.

$\mathcal{D}_{2 \text { jet }}^{V H}=\max \left(\mathcal{D}_{2 \text { jet }}^{W H}, \mathcal{D}_{2 \text { jet }}^{Z H}\right)>0.5$ using either the SM or BSM signal hypothesis for the $V H$ production.

(iii) Untagged consists of the remaining events.

The requirements on the number of $b$-tagged jets are applied to reduce crossfeed from $t \bar{t} H$ production. Even though $V H$ cross sections are significantly lower with respect to $\mathrm{VBF}$ for $m_{4 \ell}>220 \mathrm{GeV}$, the $V H$ cross section becomes comparable to the VBF cross section in the presence of anomalous couplings. Therefore, the off-shell analysis also benefits from featuring the $V H$-tagged category with hadronic decays of the associated $V$. In either the on-shell or off-shell regions, events are not tagged for the smaller $V H$ contribution with leptonic $V$ decays explicitly, but this contribution is taken into account in the simulation and parametrization of the $\mathrm{VH}$ process in the three different categories. The expected and observed numbers of events are listed in Table IV for the on-shell region and Table $\mathrm{V}$ for the off-shell region.

In each category of events, typically three observables $\vec{x}$ are defined following Eqs. (7) and (8), as summarized in Tables II and III. In the on-shell region, except for the SMlike analysis, these are $\vec{x}=\left\{\mathcal{D}_{\mathrm{bkg}}, \mathcal{D}_{a i}, \mathcal{D}_{\text {int }}\right\}$. The first observable, $\mathcal{D}_{\text {bkg }}$, is calculated differently in the three tagged categories. In the untagged category, $\mathcal{P}_{\text {bkg }}$ is calculated for the dominant $q \bar{q} \rightarrow 4 \ell$ background process. The signal and background probabilities include both the

TABLE II. Summary of the three production categories in the on-shell $m_{4 \ell}$ region. The selection requirements on the $\mathcal{D}_{2 \text { jet }}$ discriminants are quoted for each category, and further requirements can be found in the text. Two or three observables (abbreviated as obs.) are listed for each analysis and for each category. All discriminants are calculated with the JHUGEN signal matrix elements and MCFM background matrix elements. The discriminants $\mathcal{D}_{\mathrm{bkg}}$ in the tagged categories also include probabilities using associated jets and decay in addition to the $m_{4 \ell}$ probability. The $V H$ interference discriminants in the hadronic $V H$-tagged categories are defined as the simple average of the ones corresponding to the $W H$ and $Z H$ processes.

\begin{tabular}{|c|c|c|c|}
\hline Category & VBF-tagged & $V H$-tagged & Untagged \\
\hline Selection & $\mathcal{D}_{2 \text { jet }}^{\mathrm{VBF}}$ or $\mathcal{D}_{2 \mathrm{jet}}^{\mathrm{VBF}, \mathrm{BSM}}>0.5$ & $\begin{array}{c}\mathcal{D}_{2 \text { jet }}^{W H} \text { or } \mathcal{D}_{2 \mathrm{jet}}^{W H, \mathrm{BSM}} \text {, or } \\
\mathcal{D}_{2 \mathrm{jet}}^{Z H} \text { or } \mathcal{D}_{2 \mathrm{jet}}^{Z H, \mathrm{BSM}}>0.5\end{array}$ & Rest of events \\
\hline SM obs. & $m_{4 \ell}, \mathcal{D}_{\mathrm{bkg}}^{\mathrm{VBF}+\mathrm{dec}}$ & $m_{4 \ell}, \mathcal{D}_{\mathrm{bkg}}^{V H+\mathrm{dec}}$ & $m_{4 \ell}, \mathcal{D}_{\mathrm{bkg}}^{\mathrm{kin}}$ \\
\hline$a_{3}$ obs. & $\mathcal{D}_{\text {bkg }}, \mathcal{D}_{0-}^{\mathrm{VBF}+\mathrm{dec}}, \mathcal{D}_{C P}^{\mathrm{VBF}}$ & $\mathcal{D}_{\text {bkg }}, \mathcal{D}_{0-}^{V H+\mathrm{dec}}, \mathcal{D}_{C P}^{V H}$ & $\mathcal{D}_{\text {bkg }}, \mathcal{D}_{0-}^{\mathrm{dec}}, \mathcal{D}_{C P}^{\mathrm{dec}}$ \\
\hline$a_{2}$ obs. & $\mathcal{D}_{\text {bkg }}, \mathcal{D}_{0 h+}^{\mathrm{VBF}+\mathrm{dec}}, \mathcal{D}_{\text {int }}^{\mathrm{VBF}}$ & $\mathcal{D}_{\text {bkg }}, \mathcal{D}_{0 h+}^{V H+\text { dec }}, \mathcal{D}_{\text {int }}^{V H}$ & $\mathcal{D}_{\mathrm{bkg}}, \mathcal{D}_{0 h+}^{\mathrm{dec}}, \mathcal{D}_{\mathrm{int}}^{\mathrm{dec}}$ \\
\hline$\Lambda_{1}$ obs. & $\mathcal{D}_{\text {bkg }}, \mathcal{D}_{\Lambda 1}^{\mathrm{VBF}+\mathrm{dec}}, \mathcal{D}_{0 h+}^{\mathrm{VBF}+\mathrm{dec}}$ & $\mathcal{D}_{\mathrm{bkg}}, \mathcal{D}_{\Lambda 1}^{V H+\mathrm{dec}}, \mathcal{D}_{0 h+}^{V H+\mathrm{dec}}$ & $\mathcal{D}_{\text {bkg }}, \mathcal{D}_{\Lambda 1}^{\text {dec }}, \mathcal{D}_{0 h+}^{\text {dec }}$ \\
\hline$\Lambda_{1}^{Z \gamma}$ obs. & $\mathcal{D}_{\text {bkg }}, \mathcal{D}_{\Lambda 1}^{Z \gamma, \mathrm{VBF}+\mathrm{dec}}, \mathcal{D}_{0 h+}^{\mathrm{VBF}+\mathrm{dec}}$ & $\mathcal{D}_{\mathrm{bkg}}, \mathcal{D}_{\Lambda 1}^{Z \gamma, V H+\mathrm{dec}}, \mathcal{D}_{0 h+}^{V H+\mathrm{dec}}$ & $\mathcal{D}_{\text {bkg }}, \mathcal{D}_{\Lambda 1}^{Z \gamma, \mathrm{dec}}, \mathcal{D}_{0 h+}^{\mathrm{dec}}$ \\
\hline
\end{tabular}


TABLE III. Summary of the three production categories in the off-shell $m_{4 \ell}$ region, listed in a similar manner, as in Table II. All discriminants are calculated with the JHUGEN or MCFM/JHUGEN signal, and MCFM background matrix elements. The $V H$ interference discriminant in the SM-like analysis hadronic $V H$-tagged category is defined as the simple average of the ones corresponding to the $W H$ and $\mathrm{ZH}$ processes.

\begin{tabular}{|c|c|c|c|}
\hline Category & VBF-tagged & $V H$-tagged & Untagged \\
\hline Selection & $\mathcal{D}_{2 \text { jet }}^{\mathrm{VBF}}$ or $\mathcal{D}_{2 \mathrm{jet}}^{\mathrm{VBF}, \mathrm{BSM}}>0.5$ & $\begin{array}{c}\mathcal{D}_{2 \text { jet }}^{W H} \text { or } \mathcal{D}_{2 \text { jet }}^{W H, \mathrm{BSM}} \text {, or } \\
\mathcal{D}_{2 \mathrm{jet}}^{Z H} \text { or } \mathcal{D}_{2 \mathrm{jet}}^{Z H, \mathrm{BSM}}>0.5\end{array}$ & Rest of events \\
\hline SM obs. & $m_{4 \ell}, \mathcal{D}_{\mathrm{bkg}}^{\mathrm{VBF}+\mathrm{dec}}, \mathcal{D}_{\mathrm{bsi}}^{\mathrm{VBF}+\mathrm{dec}}$ & $m_{4 \ell}, \mathcal{D}_{\mathrm{bkg}}^{V H+\mathrm{dec}}, \mathcal{D}_{\mathrm{bsi}}^{V H+\mathrm{dec}}$ & $m_{4 \ell}, \mathcal{D}_{\mathrm{bkg}}^{\mathrm{kin}}, \mathcal{D}_{\mathrm{bsi}}^{g g, \mathrm{dec}}$ \\
\hline$a_{3}$ obs. & $m_{4 \ell}, \mathcal{D}_{\mathrm{bkg}}^{\mathrm{VBF}+\mathrm{dec}}, \mathcal{D}_{0-}^{\mathrm{VBF}+\mathrm{dec}}$ & $m_{4 \ell}, \mathcal{D}_{\mathrm{bkg}}^{V H+\mathrm{dec}}, \mathcal{D}_{0-}^{V H+\mathrm{dec}}$ & $m_{4 \ell}, \mathcal{D}_{\mathrm{bkg}}^{\mathrm{kin}}, \mathcal{D}_{0-}^{\mathrm{dec}}$ \\
\hline$a_{2}$ obs. & $m_{4 \ell}, \mathcal{D}_{\mathrm{bkg}}^{\mathrm{VBF}+\mathrm{dec}}, \mathcal{D}_{0 h+}^{\mathrm{VBF}+\mathrm{dec}}$ & $m_{4 \ell}, \mathcal{D}_{\mathrm{bkg}}^{V H+\mathrm{dec}}, \mathcal{D}_{0 h+}^{V H+\mathrm{dec}}$ & $m_{4 \ell}, \mathcal{D}_{\mathrm{bkg}}^{\mathrm{kin}}, \mathcal{D}_{0 h+}^{\mathrm{dec}}$ \\
\hline$\Lambda_{1}$ obs. & $m_{4 \ell}, \mathcal{D}_{\mathrm{bkg}}^{\mathrm{VBF}+\mathrm{dec}}, \mathcal{D}_{\Lambda 1}^{\mathrm{VBF}+\mathrm{dec}}$ & $m_{4 \ell}, \mathcal{D}_{\mathrm{bkg}}^{V H+\mathrm{dec}}, \mathcal{D}_{\Lambda 1}^{V H+\mathrm{dec}}$ & $m_{4 \ell}, \mathcal{D}_{\mathrm{bkg}}^{\mathrm{kin}}, \mathcal{D}_{\Lambda 1}^{\mathrm{dec}}$ \\
\hline
\end{tabular}

TABLE IV. The numbers of events expected in the SM (or $f_{a 3}=1$ in parentheses) for the different signal and background contributions and the total numbers of observed events are listed across the three $a_{3}$ analysis categories in the on-shell region for the combined 2016 and 2017 data set.

\begin{tabular}{lccc}
\hline \hline & VBF-tagged & $V H$-tagged & Untagged \\
\hline VBF signal & $4.7(3.4)$ & $0.3(0.2)$ & $5.7(0.8)$ \\
$W H$ signal & $0.3(0.6)$ & $0.7(1.9)$ & $2.1(5.3)$ \\
$Z H$ signal & $0.2(0.4)$ & $0.5(1.0)$ & $1.5(2.5)$ \\
VV background & 0.2 & 0.1 & 0.5 \\
$g g$ signal & $5.5(5.8)$ & $3.2(3.3)$ & $98.9(98.4)$ \\
$g g$ background & 0.8 & 0.3 & 12.7 \\
$t \bar{t} H$ signal & $0.2(0.2)$ & $0.1(0.1)$ & $1.1(1.2)$ \\
$b \bar{b} H$ signal & $0.1(0.1)$ & $0.1(0.1)$ & $1.1(1.1)$ \\
$q \bar{q} \rightarrow 4 \ell$ background & 1.6 & 1.5 & 120.3 \\
$Z+X$ background & 5.2 & 3.0 & 46.3 \\
Total expected & $18.8(18.2)$ & $9.7(11.4)$ & $290.3(289.1)$ \\
Total observed & 19 & 9 & 332 \\
\hline \hline
\end{tabular}

matrix element probability based on the four-lepton kinematics and the $m_{4 \ell}$ probability parametrization extracted from simulation of detector effects. The signal $m_{4 \ell}$ parametrization assumes $m_{H}=125 \mathrm{GeV}$. In the VBF-tagged and $V H$-tagged categories, $\mathcal{P}_{\text {bkg }}$ and $\mathcal{P}_{\text {sig }}$ include fourlepton kinematics and the $m_{4 \ell}$ probability parametrization, but they also include kinematics of the two associated jets. The $\mathcal{P}_{\text {bkg }}$ probability density represents the EW and QCD

TABLE V. The numbers of events expected in the SM-like analysis (or $f_{a 3}=0$ in the $a_{3}$ analysis categorization, divided with a vertical bar) for the different signal and background contributions and the total observed numbers of events are listed across the three SM $\mid a_{3}$ analysis categories in the off-shell region for the combined 2016 and 2017 data set. The signal, background, and interference contributions are shown separately for the gluon fusion $(g g)$ and EW processes (VV) under the $\Gamma_{H}=\Gamma_{H}^{\mathrm{SM}}$ assumption.

\begin{tabular}{|c|c|c|c|}
\hline & VBF-tagged & $V H$-tagged & Untagged \\
\hline VV signal & $1.0 \mid 1.2$ & $0.3 \mid 0.3$ & $3.3 \mid 3.1$ \\
\hline VV background & $7.3 \mid 9.9$ & $2.5 \mid 2.8$ & $16.2 \mid 13.3$ \\
\hline VV interference & $-1.8 \mid-2.1$ & $0.06 \mid 0.03$ & $-2.4 \mid-2.2$ \\
\hline$g g$ signal & $1.0 \mid 1.6$ & $0.8 \mid 1.0$ & $20.3 \mid 19.5$ \\
\hline$g g$ background & $10.4 \mid 16.4$ & $8.7 \mid 10.4$ & $245.9 \mid 238.1$ \\
\hline$g g$ interference & $-1.6 \mid-2.6$ & $-1.4 \mid-1.6$ & $-34.4 \mid-33.0$ \\
\hline$q \bar{q} \rightarrow 4 \ell$ background & $15.8 \mid 33.5$ & $27.8 \mid 31.2$ & $992.0 \mid 970.8$ \\
\hline$Z+X$ background & $2.4 \mid 6.4$ & $2.8 \mid 3.3$ & $45.4 \mid 40.8$ \\
\hline Total expected & $34.4 \mid 64.8$ & $41.6 \mid 47.5$ & $1286.3 \mid 1251.0$ \\
\hline Total observed & $36 \mid 92$ & $46 \mid 51$ & $1325 \mid 1264$ \\
\hline
\end{tabular}



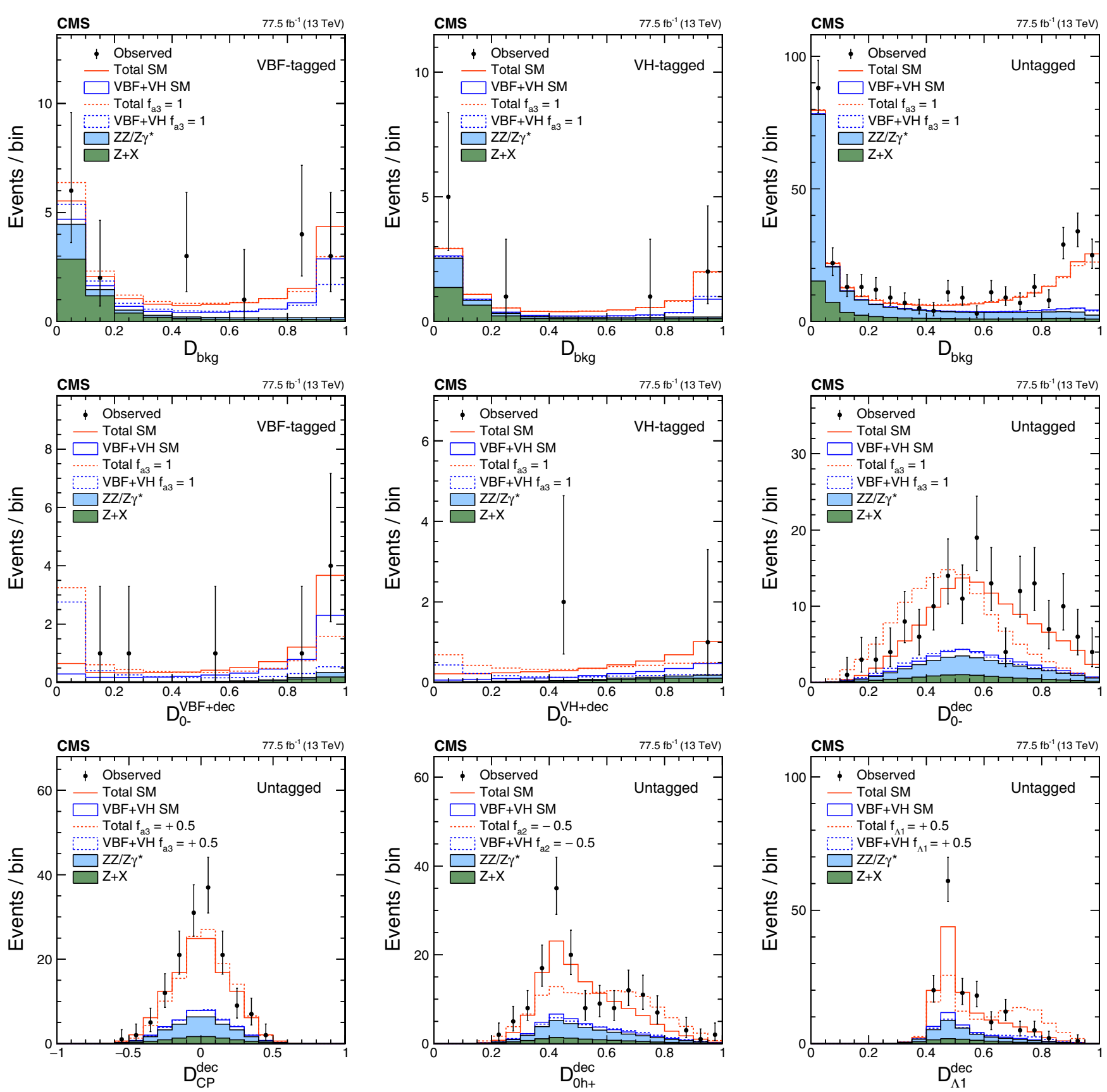

FIG. 3. The distributions of events in the on-shell region in the data from 2016 and 2017. The top row shows $\mathcal{D}_{\text {bkg }}$ in the VBF-tagged (left), $V H$-tagged (middle), and untagged (right) categories of the analysis of the $a_{3}$ coupling for a pseudoscalar contribution. The rest of the distributions are shown with the requirement $\mathcal{D}_{\text {bkg }}>0.5$ in order to enhance signal over background contributions. The middle row shows $\mathcal{D}_{0-}$ in the corresponding three categories. The bottom row shows $\mathcal{D}_{C P}^{\mathrm{dec}}$ of the $a_{3}$, $\mathcal{D}_{0 h+}^{\mathrm{dec}}$ of the $a_{2}$, and $\mathcal{D}_{\Lambda 1}^{\mathrm{dec}}$ of the $\Lambda_{1}$ analyses in the untagged categories.

background processes $4 \ell+2$ jets, while $\mathcal{P}_{\text {sig }}$ represents EW processes $\mathrm{VBF}$ and $\mathrm{VH}$. It was found that jet kinematics in the $\mathcal{D}_{\text {bkg }}$ calculation improves separation of the targeted signal production both against background and against the $H$ boson gluon fusion production. However, in the off-shell region and in the SM-like on-shell analysis, the four-lepton invariant mass $m_{4 \ell}$ is one of the most important observables, because the mass parametrization becomes an important feature of the analysis. Therefore, the $m_{4 \ell}$ parametrization is not used in the $\mathcal{D}_{\text {bkg }}$ calculation in these cases, and this is reflected with the superscript denoting which information is used, either with decay only information in $\mathcal{D}_{\text {bkg }}^{\text {kin }}$ or with both decay and production in $\mathcal{D}_{\text {bkg }}^{\mathrm{VBF}+\text { dec }}$ and $\mathcal{D}_{\text {bkg }}^{V H+\text { dec }}$.

The other observable, $\mathcal{D}_{a i}$, separates the SM hypothesis $f_{a i}=0$ as $\mathcal{P}_{\text {sig }}$ from the alternative hypothesis $f_{a i}=1$ as $\mathcal{P}_{\text {alt }}$, following Eq. (7). In the untagged category, the 

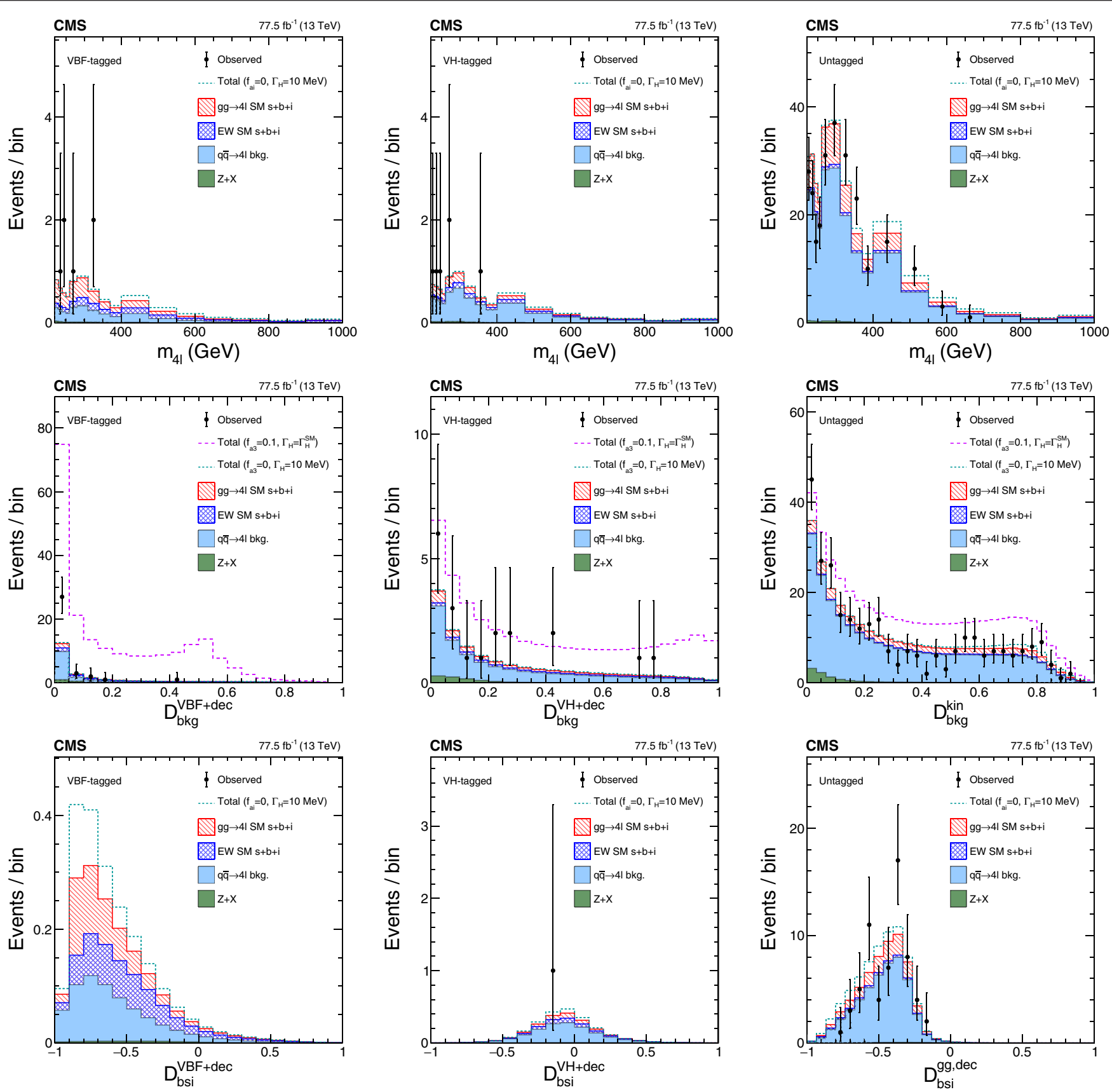

FIG. 4. The distributions of events in the off-shell region in the data from 2016 and 2017. The top row shows $m_{4 \ell}$ in the VBF-tagged (left), $V H$-tagged (middle), and untagged (right) categories in the dedicated SM-like width analysis where a requirement on $\mathcal{D}_{\text {bkg }}^{\mathrm{VBF}+\text { dec }}$, $\mathcal{D}_{\text {bkg }}^{V H+\text { dec }}$, or $\mathcal{D}_{\text {bkg }}^{\text {kin }}>0.6$ is applied in order to enhance signal over background contributions. The middle row shows $\mathcal{D}_{\text {bkg }}^{\mathrm{VBF}+\mathrm{dec}}$ (left), $\mathcal{D}_{\mathrm{bkg}}^{V H+\mathrm{dec}}$ (middle), $\mathcal{D}_{\mathrm{bkg}}^{\mathrm{kin}}$ (right) of the $a_{3}$ analysis in the corresponding three categories. The requirement $m_{4 \ell}>340 \mathrm{GeV}$ is applied in order to enhance signal over background contributions. The bottom row shows $\mathcal{D}_{\text {bsi }}$ in the corresponding three categories in the dedicated SM-like width analysis with both of the $m_{4 \ell}$ and $\mathcal{D}_{\mathrm{bkg}}^{\mathrm{kin}}$ requirements enhancing the signal contribution. The acronym $\mathrm{s}+\mathrm{b}+\mathrm{i}$ designates the sum of the signal (s), background (b), and their interference contributions (i).

probabilities are calculated using only the decay information, and the $\mathcal{D}_{a i}$ observable is called $\mathcal{D}_{0-}$ in the $a_{3}, \mathcal{D}_{\text {oh }+}$ in the $a_{2}, \mathcal{D}_{\Lambda 1}$ in the $\Lambda_{1}$, and $\mathcal{D}_{\Lambda 1}^{Z_{\gamma}}$ in the $\Lambda_{1}^{Z_{\gamma}}$ analyses [25]. In the VBF-tagged and $V H$-tagged categories, both the production and decay probabilities are used, with the matrix elements calculated as the product of the decay component and the component from either VBF production or $(W H+Z H)$ associated production, respectively [27]. The resultant set of $\mathcal{D}_{a i}$ discriminants are called in a similar manner to their counterparts in the untagged category but indicating the production assumption in their upper index. 
The last observable, $\mathcal{D}_{\text {int }}$ defined in Eq. (8), separates the interference of the two amplitudes corresponding to the SM-like $H$ boson coupling and the alternative $H$ boson coupling model, or the SM-like $H$ boson coupling and background as an alternative model in the case of $\mathcal{D}_{\text {bsi }}$ for the signal-background interference in the off-shell region. In the case of the $a_{3}$ analysis, this observable is called $\mathcal{D}_{C P}$ because if $C P$ is violated it would exhibit a distinctive forward-backward asymmetry. In the untagged category, decay information is used in the calculation of $\mathcal{D}_{\text {int. }}$. In the VBF-tagged and $V H$-tagged categories, production information with the two associated jets is used. The $\mathcal{D}_{\text {bsi }}$ discriminant extends the idea of the $\mathcal{D}_{g g}$ discriminant introduced in Ref. [11] for the $H$ boson width measurement, but allows independent treatment of the interference component. It is used only in the SM-like analysis.

The distributions of events for several of the observables $\vec{x}$ from Tables II and III are illustrated in Fig. 3 for the onshell and in Fig. 4 for the off-shell regions. In Figs. 3 and 4, cross sections of all background processes are fixed to the SM expectations, except for the $Z+X$ background estimated from the data control regions discussed above. Cross sections of all signal processes, including BSM, are normalized to the SM expectations in the on-shell region.

\section{THE FIT IMPLEMENTATION}

We perform an unbinned extended maximum likelihood fit [89] to the events split into several categories (enumerated with an index $k$ below) according to the three lepton flavor combinations $(4 e, 4 \mu$, and $2 e 2 \mu)$, three production categories (VBF-tagged, $V H$-tagged, and untagged), five data periods (2011, 2012, 2015, 2016, and 2017), and two mass ranges (on-shell and off-shell). Therefore, there could be up to 90 categories of events. However, not all categories are used in each independent measurement because of the simpler categorization approach applied to the earlier data. Here we focus on discussion of the 2016 and 2017 data analyses, while treatment of the earlier data can be found in Refs. [13,25,27].

An independent fit is performed for each of the four anomalous $H V V$ coupling parameters $f_{a i} \cos \left(\phi_{a i}\right)$ using the on-shell region only. These fits avoid any assumptions on how the behavior of each process considered in the analysis changes from the on-shell region to the off-shell region. Four independent joint fits to the on-shell and offshell regions are performed in order to determine the width of the $H$ boson under the SM-like assumption or in the presence of the three anomalous couplings $a_{3}, a_{2}$, and $\Lambda_{1}$. These fits are also used to constrain the three corresponding anomalous coupling parameters $f_{a i} \cos \left(\phi_{a i}\right)$. When a certain anomalous coupling is tested, all other anomalous couplings are assumed to be zero, and only real couplings in Eq. (2) are tested, that is with $a_{1} \geq 0$ and $\cos \left(\phi_{a i}\right)= \pm 1$.

The on-shell analysis with the study of the $a_{3}, a_{2}, \Lambda_{1}$, and $\Lambda_{1}^{Z \gamma}$ couplings has been presented in Ref. [27] using a partial data set. This part of the analysis remains essentially unchanged, except for a small change in the definition of the interference discriminant in Eq. (8) and the inclusion of information from the kinematics of the two associated jets in the $\mathcal{D}_{\text {bkg }}$ calculation discussed in Sec. IV. The SM-like on-shell analysis is similar to the one presented in Ref. [21] in methodology, but it uses the observables $\vec{x}$ and categorization $k$ described in Table II and Sec. IV. The on-shell probability density is normalized to the total event yield in each process $j$ and category $k$ according to

$\mathcal{P}_{j k}\left(\vec{x} ; \vec{\xi}_{j k}, \vec{\zeta}\right)=\mu_{j} \mathcal{P}_{j k}^{\mathrm{sig}}\left(\vec{x} ; \vec{\xi}_{j k}, f_{a i}, \phi_{a i}\right)+\mathcal{P}_{j k}^{\mathrm{bkg}}\left(\vec{x} ; \vec{\xi}_{j k}\right)$,

where $\vec{\zeta}=\left(\mu_{F}, \mu_{V}, \Gamma_{H}, f_{a i} \cos \left(\phi_{a i}\right)\right)$ are the unconstrained parameters of interest, $\vec{\xi}_{j k}$ are the constrained nuisance parameters for a particular parametrization, and $\vec{x}$ are the observables listed in Table II, specific to each $a_{i}$. The onshell signal strength $\mu_{j}$ in Eq. (9) is defined in references to Eq. (1) as either $\mu_{F}$ or $\mu_{V}$ according to the process type $j$ ( $g g, \mathrm{VBF}, W H, Z H, t \bar{t} H, b \bar{b} H, q \bar{q} \rightarrow 4 \ell$, and $Z+X$ ). Each process includes both signal (sig) and background (bkg) components, but may contain only signal $(\bar{t} \bar{t} H$ and $b \bar{b} H)$ or only background $(q \bar{q} \rightarrow 4 \ell$ and $Z+X)$ contributions in the particular cases. The interference between the signal and background components, when both are present, is negligible in the on-shell region because of the very small width $\Gamma_{H}$ compared to the mass range of interest. This also leads to the on-shell parametrization in Eq. (9) being independent from the width $\Gamma_{H}$.

The off-shell probability density follows Eqs. (1) and (9) closely but with the additional contribution of interference (int) between the signal and background amplitudes as

$$
\begin{aligned}
\mathcal{P}_{j k}\left(\vec{x} ; \vec{\xi}_{j k}, \vec{\zeta}\right)= & \frac{\mu_{j} \Gamma_{H}}{\Gamma_{0}} \mathcal{P}_{j k}^{\mathrm{sig}}\left(\vec{x} ; \vec{\xi}_{j k}, f_{a i}, \phi_{a i}\right) \\
& +\sqrt{\frac{\mu_{j} \Gamma_{H}}{\Gamma_{0}}} \mathcal{P}_{j k}^{\mathrm{int}}\left(\vec{x} ; \vec{\xi}_{j k}, f_{a i}, \phi_{a i}\right) \\
& +\mathcal{P}_{j k}^{\mathrm{bkg}}\left(\vec{x} ; \vec{\xi}_{j k}\right),
\end{aligned}
$$

where the notation remains the same as for Eq. (9). The $\vec{x}$ observables are listed in Table III and are specific to each coupling analysis. They include $m_{4 \ell}$ and two other discriminants. The process type $j$ does not include $t \bar{t} H$ and $b \bar{b} H$ because of their negligible contribution in the off-shell region, while the $\mathrm{VBF}, W H$, and $\mathrm{ZH}$ processes are combined into one EW process. The parametrization in Eq. (10) depends on the width $\Gamma_{H}$ explicitly and the reference value is taken to be $\Gamma_{0}=4.07 \mathrm{MeV}$, which determines the relative strength of $\mathcal{P}_{j k}^{\text {sig }}$ and $\mathcal{P}_{j k}^{\text {int }}$ with respect to $\mathcal{P}_{j k}^{\mathrm{bkg}}$ in the parametrization.

The EW $H$ boson production (VBF and $V H$ ) or production via gluon fusion have different dependence on 

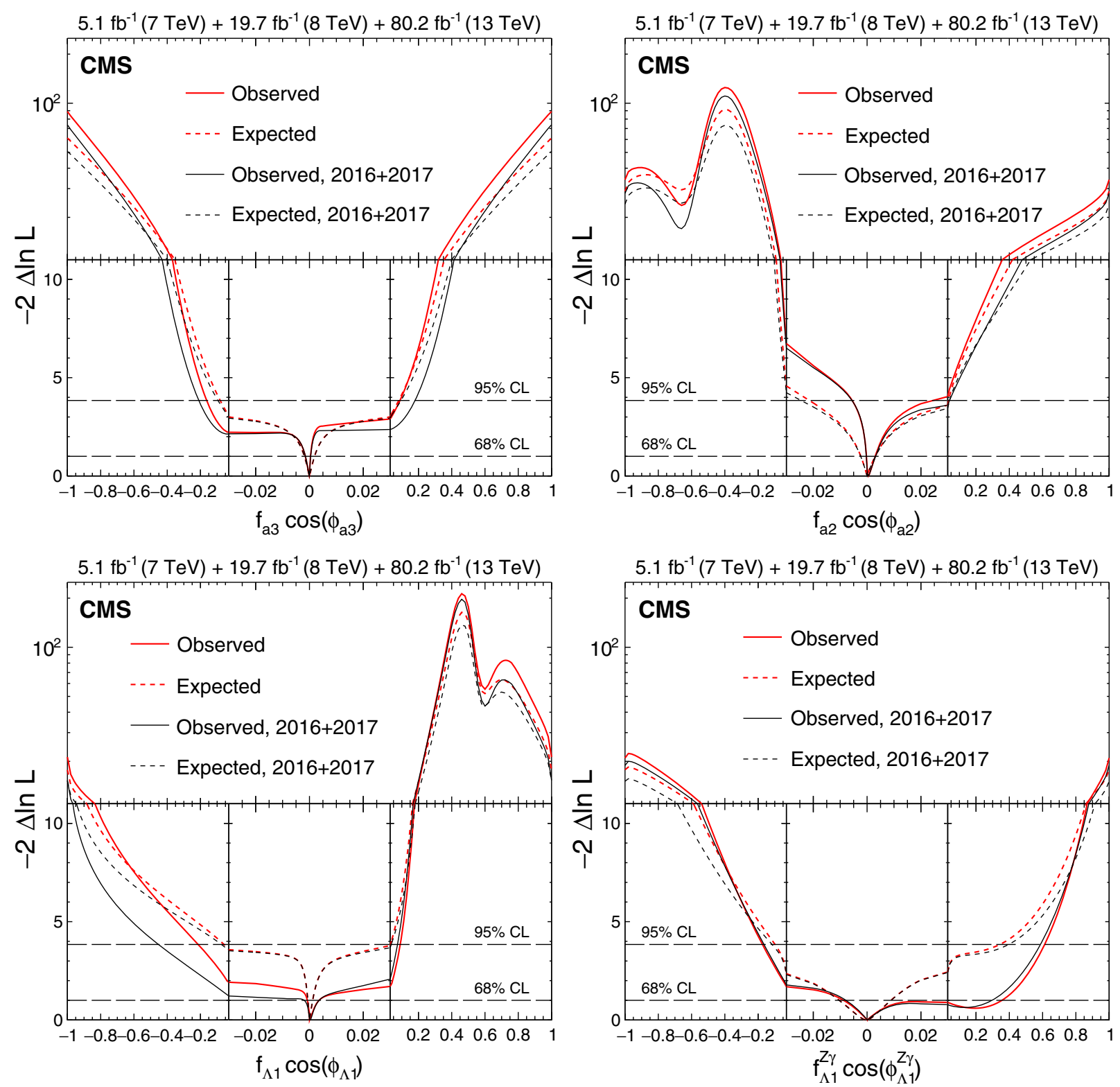

FIG. 5. Observed (solid) and expected (dashed) likelihood scans of $f_{a 3} \cos \left(\phi_{a 3}\right)$ (top left), $f_{a 2} \cos \left(\phi_{a 2}\right)$ (top right), $f_{\Lambda 1} \cos \left(\phi_{\Lambda 1}\right)$ (bottom left), and $f_{\Lambda 1}^{Z_{\gamma}} \cos \left(\phi_{\Lambda 1}^{Z_{\gamma}}\right)$ (bottom right) using on-shell events only. Results of analysis of the data from 2016 and 2017 only (black) and the combined Run 1 and Run 2 analysis (red) are shown. The dashed horizontal lines show the 68 and 95\% C.L. regions.

anomalous $H V V$ couplings, equally in the on-shell or offshell regions. There are two $H V V$ vertices in the former production mechanism with the subsequent $H \rightarrow V V \rightarrow 4 \ell$ decay while there is only one $H V V$ decay vertex in the latter case. In addition, there is interference with the background in the off-shell region. This leads to the following general expressions for the signal (sig) or interference (int) contributions appearing in Eqs. (9) and (10):

$$
\begin{aligned}
\mathcal{P}_{j k}^{\mathrm{sig} / \mathrm{int}}\left(\vec{x} ; \vec{\xi}_{j k}, f_{a i}, \phi_{a i}\right) \\
\quad=\sum_{m=0}^{M} \mathcal{P}_{j k, m}^{\mathrm{sig} / \mathrm{int}}\left(\vec{x} ; \vec{\xi}_{j k}\right) f_{a i}^{\frac{m}{2}}\left(1-f_{a i}\right)^{\frac{M-m}{2}} \cos ^{m}\left(\phi_{a i}\right),
\end{aligned}
$$

where the sum over the index $m$ runs up to $M=4$ in the case of the EW signal process; $M=2$ in the case of the gluon fusion, $t \bar{t} H$, and $b \bar{b} H$ signal processes, or the interference between the signal and background in the EW process; and $M=1$ in the case of the interference between the signal and background in the gluon fusion process. In this expression, the index $m$ corresponds to the exponent of $a_{i}$ in the squared scattering amplitude from Eq. (2), which may contain contributions from production and decay, and the factor $\cos \left(\phi_{a i}\right)= \pm 1$ affects only the sign of the terms that scale with an odd power of $a_{i}$.

The $\mathcal{P}_{j k, m}^{\mathrm{sig} / \mathrm{int}}$ and $\mathcal{P}_{j k}^{\mathrm{bkg}}$ probability densities are normalized to the expected number of events, and are binned 
histograms (templates) of the observables $\vec{x}$ listed in Tables II and III, except for the signal $m_{4 \ell}$ parametrization in the on-shell region as discussed below. These templates are obtained by reweighting the existing signal or background samples for different couplings and then finding their linear combination. Since $m_{4 \ell}$ is treated directly as an observable in the on-shell SM-like fit, the signal $m_{4 \ell}$ shape for each process $j$ and category $k$ is parametrized using a double-sided crystal-ball function [90], and the full signal probability density is parametrized as the product of the parametric $m_{4 \ell}$ shape and a template of other discriminants conditional in $m_{4 \ell}$. In all cases, the $H$ boson mass $m_{H}=$ $125 \mathrm{GeV}$ is assumed.

The final constraints on $f_{a i} \cos \left(\phi_{a i}\right)$ and $\Gamma_{H}$ are placed using the profile likelihood method using the RooFit toolkit [91] within the ROOT [92] framework. The extended likelihood function is constructed using the probability densities in Eqs. (9) and (10) with each event characterized by the discrete category $k$ and typically three continuous observables $\vec{x}$. The likelihood $\mathcal{L}$ is maximized with respect to the nuisance parameters $\vec{\xi}_{j k}$ describing the systematic uncertainties discussed below and the yield parameters $\mu_{F}$ and $\mu_{V}$. The allowed $68 \%$ and $95 \%$ C.L. intervals are defined using the profile likelihood function, $-2 \Delta \ln \mathcal{L}=$ 1.00 and 3.84 , for which exact coverage is expected in the asymptotic limit [93].

Several systematic uncertainties are featured in the vectors of constrained parameters $\vec{\xi}_{j k}$. The template shapes describing probability distributions in Eqs. (9), (10), and (11) are varied separately within either theoretical or experimental uncertainties. In the following, a range of uncertainties affecting the template distributions is given for the $m_{4 \ell}$ values from around $100 \mathrm{GeV}$ (typical for the onshell range) to around $1 \mathrm{TeV}$ (in the off-shell range), respectively. The factorization (or renormalization) scale uncertainties are evaluated by multiplying the central scale by 2 or $1 / 2$, and the uncertainties range from $\pm 0.7 \%$ $\left({ }_{-1.4}^{+1.2} \%\right)$ to ${ }_{+0.6}^{-1.0} \%\left({ }_{-4}^{+5} \%\right)$ in the $g g$ process, from ${ }_{-0.1}^{+0.6} \%$ $\left({ }_{-4}^{+5} \%\right)$ to $\pm 5 \%\left({ }_{-25}^{+30} \%\right)$ in the VBF process, from ${ }_{-5}^{+3} \%$ $\left({ }_{-4}^{+5} \%\right)$ to $\pm 6 \%\left({ }_{-25}^{+30} \%\right)$ in the processes with an associated EW boson, and from ${ }_{-5.5}^{+3.5} \%$ to $\pm 1 \%( \pm 3 \%)$ in the $q \bar{q} \rightarrow 4 \ell$ background. PDF parametrization uncertainties are evaluated by taking the envelope of the 100 alternative NNPDF variations. Variations due to PDF parametrization uncertainties [or due to uncertainties in $\alpha_{S}\left(m_{Z}\right)=0.1180 \pm$ 0.0015] range from ${ }_{-1.4}^{+1.2} \%\left({ }_{-2.5}^{+2.0} \%\right)$ to ${ }_{-4}^{+5} \%\left({ }_{-1.0}^{+2.4} \%\right)$ in the $g g$ process, from ${ }_{-4}^{+5} \%$ to about ${ }_{-25}^{+30} \%$ in the EW processes, and are approximately $\pm 3 \%$ (from ${ }_{-1.8}^{+1.0} \%$ to $\pm 0.5 \%$ ) for the $q \bar{q} \rightarrow 4 \ell$ background. The signal processes, and the backgrounds that interfere with the signal, feature the uncertainties as a function of the multiplicity and kinematics of associated jets due to the hadronization scale used in PYTHIA and the underlying event variations, obtained with the variations of the PYTHIA tune. In the VBF-tagged categories, the correlated template variations for the hadronization scale (underlying event) range from $\pm 11 \%$ ( $\pm 45 \%)$ to $\mp 8 \%$ ( $\mp 40 \%)$ in the $g g$ process, from $\pm 8 \%( \pm 24 \%)$ to $\mp 6 \%$ ( $\mp 8 \%)$ in the VBF process, and from $\pm 13 \%( \pm 20 \%)$ to $\mp 10 \%$ ( $\mp 32 \%)$ in the processes with an associated EW boson. In the $V H$-tagged categories, these correlated template variations instead range from $\pm 15 \%( \pm 50 \%)$ to $\mp 9 \%$ ( $\mp 45 \%)$ in the $g g$ process, from $\pm 8 \%( \pm 25 \%)$ to $\mp 7 \%$ ( $\mp 30 \%)$ in the VBF process, and from $\pm 4 \%( \pm 19 \%)$ to $\mp 4 \%(\mp 13 \%)$ in the processes with an associated EW boson. Template shapes in the $g g$ processes are also varied to account for a second jet in the hard process, and these correlated variations range from $\pm 18 \%( \pm 32 \%)$ to $\mp 15 \%$ ( $\mp 14 \%)$ in the VBF-tagged ( $V H$ tagged) category. The $q \bar{q} \rightarrow 4 \ell$ background further features an uncertainty in the NLO EW corrections applied to the simulation $[79,80]$, which are significant at higher $m_{4 \ell}$ values, reaching up to $20 \%$ at $1 \mathrm{TeV}$.

Experimental uncertainties involve jet energy calibration (JEC) uncertainties, which are only relevant when production categories are considered, and lepton efficiency and momentum uncertainties, which are similar for the different processes and categories. Systematic uncertainties in the JEC account for variations in the VBF-tagged ( $\mathrm{VH}$-tagged) category, and range from $\pm 13 \%( \pm 4 \%)$ to $\pm 8 \%( \pm 1 \%)$ in the $g g$ process, from $\pm 5 \%\left({ }_{+2}^{-10} \%\right)$ to about $\pm 11 \%( \pm 6 \%)$ in the VBF process, from $\pm 9 \%$ ( $\pm 4 \%)$ to $\pm 12 \%$ ( $\pm 1 \%$ ) in processes with an associated EW boson, and from $\pm 17 \%$ $( \pm 8 \%)$ to $\pm 15 \%\left({ }_{-0.5}^{+2.0} \%\right)$ for the $q \bar{q} \rightarrow 4 \ell$ background. The cross-section uncertainties due to electron (muon) efficiency range from ${ }_{-7}^{+6} \%\left({ }_{-4.5}^{+3.0} \%\right)$ to ${ }_{-4.5}^{+3.5} \%\left({ }_{-2.0}^{+0.8} \%\right)$ to ${ }_{-8}^{+7} \%$ $\left({ }_{-2.0}^{+0.8} \%\right)$ in the $2 e 2 \mu$ channel, and roughly double for the $4 e$

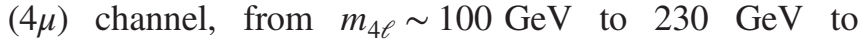
around $1 \mathrm{TeV}$.

In the estimation of the $Z+X$ background, the flavor composition of hadronic jets misidentified as leptons may be different in the $Z+1 \ell$ and $Z+2 \ell$ control regions, and together with the statistical uncertainty in the $Z+2 \ell$ region, this uncertainty accounts for about $\pm 30 \%$ variation in the background estimate from the 2017 data set. The uncertainty on the modeling of this misidentification as a function of $p_{T}$ and $\eta$, combined with the $Z+1 \ell$ control region statistical uncertainty, leads to a ${ }_{-12}^{+20} \%$ to ${ }_{-27}^{+30} \%$ variation in the $4 e$ channel, $\pm(10-20) \%$ variation in the $m_{4 \ell}$ shape in the $2 e 2 \mu$ channel, and $\pm 4 \%$ to ${ }_{-17}^{+14} \%$ variation in the $4 \mu$ channel. Uncertainties in the $Z+X$ background in the 2016 data set are only slightly larger. The normalization of the background processes derived from the MC simulation is affected by the uncertainties in the integrated luminosity of $2.5 \%$ [94] and $2.3 \%$ [95] in the 2016 and 2017 data sets, respectively. The integrated luminosity is measured using data from the CMS silicon pixel detector, drift tubes, and the forward hadron calorimeters, or from 
TABLE VI. Summary of allowed $68 \%$ C.L. (central values with uncertainties) and 95\% C.L. (in square brackets) intervals for the anomalous coupling parameters $f_{a i} \cos \left(\phi_{a i}\right)$ obtained from the analysis of the combination of Run 1 (only on-shell) and Run 2 (on-shell and off-shell) data sets. Three constraint scenarios are shown: using only on-shell events, using both on-shell and off-shell events with the $\Gamma_{H}$ left unconstrained, or with the constraint $\Gamma_{H}=\Gamma_{H}^{\mathrm{SM}}$.

\begin{tabular}{lccc}
\hline \hline Parameter & Scenario & Observed & Expected \\
\hline$f_{a 3} \cos \left(\phi_{a 3}\right)$ & On-shell & $-0.0001_{-0.0015}^{+0.0004}[-0.163,0.090]$ & $0.0000_{-0.0019}^{+0.0019}[-0.082,0.082]$ \\
& Any $\Gamma_{H}$ & $0.0000_{-0.0010}^{+0.003}[-0.0165,0.0087]$ & $0.0000_{-0.0015}^{+0.0015}[-0.038,0.038]$ \\
$f_{a 2} \cos \left(\phi_{a 2}\right)$ & $\Gamma_{H}=\Gamma_{H}^{\mathrm{SM}}$ & $0.0000_{-0.0009}^{+0.0003}[-0.0067,0.0050]$ & $0.0000_{-0.0014}^{+0.0014}[-0.0098,0.0098]$ \\
& On-shell & $0.0004_{-0.0006}^{+0.002}[-0.0055,0.0234]$ & $0.0000_{-0.0023}^{+0.0030}[-0.021,0.035]$ \\
& Any $\Gamma_{H}$ & $0.0004_{-0.0006}^{+0.0026}[-0.0035,0.0147]$ & $0.0000_{-0.0017}^{+0.0019}[-0.015,0.021]$ \\
$f_{\Lambda 1} \cos \left(\phi_{\Lambda 1}\right)$ & $\Gamma_{H}=\Gamma_{H}^{\mathrm{SM}}$ & $0.0005_{-0.0006}^{+0.0025}[-0.0029,0.0129]$ & $0.0000_{-0.0016}^{+0.0012}[-0.010,0.012]$ \\
& On-shell & $0.0002_{-0.0009}^{+0.0030}[-0.209,0.089]$ & $0.0000_{-0.0006}^{+0.0012}[-0.059,0.032]$ \\
$f_{\Lambda 1}^{Z \gamma} \cos \left(\phi_{\Lambda 1}^{Z \gamma}\right)$ & Any $\Gamma_{H}$ & $0.0001_{-0.0006}^{+0.0015}[-0.090,0.059]$ & $0.0000_{-0.0007}^{+0.0013}[-0.017,0.019]$ \\
\hline \hline
\end{tabular}

the fast beam conditions monitor and pixel luminosity telescope. All systematic uncertainties are treated as correlated between different time periods except for the luminosity and jet-related uncertainties which originate from statistically independent sources.

\section{RESULTS}

Four $f_{a i} \cos \left(\phi_{a i}\right)$ parameters sensitive to anomalous $H V V$ interactions, as defined in Eqs. (2) and (6), are tested in the on-shell data sample using the probability densities defined in Eq. (9). Since only the real couplings are tested, $\cos \left(\phi_{a i}\right)= \pm 1$. Figure 5 shows the results of the likelihood scans of these parameters for the 2016 and 2017 periods of the $13 \mathrm{TeV}$ run and for the full combined data set from collisions at 7, 8, and $13 \mathrm{TeV}$. The analysis of the 2016 and 2017 data uses the approach presented here with the observables sensitive to anomalous couplings in both production and decay. Because of the smaller numbers of events, the data from the 2015 period of the $13 \mathrm{TeV}$ run and from the 2011 and 2012 periods of the 7 and $8 \mathrm{TeV}$ runs are analyzed using only the decay information as in Refs. [25,27], which is equivalent to having all events in the untagged category of this analysis. The results from onshell events in the combined data set are listed in Table VI. These results supersede our previous measurements of these parameters in Refs. [25,27].

The observed and expected $68 \%$ C.L. constraints are significantly tighter than in the Run 1 analysis [25] as it is evident from the narrow minima at $f_{a i}=0$ in Fig. 5. This effect comes from utilizing production information because the cross section in $\mathrm{VBF}$ and $V H$ production increases quickly with $f_{a i}$. Moreover, the minima of the $-2 \ln \mathcal{L}$ distributions appear rather sharp because of the higher order polynomial of the $f_{\text {ai }}$ parameters appearing in Eq. (11) in the case of VBF and $V H$ production. At the same time, the constraints above $f_{a i} \sim 0.02$ are dominated by the decay information from $H \rightarrow 4 \ell$. The best fit $\left(\mu_{F}, \mu_{V}\right)$ values in the four analyses under the assumption that $f_{a i}=0$ are as follows: $\left(1.21_{-0.17}^{+0.21}, 0.84_{-0.59}^{+0.71}\right)$ at $f_{a 3}=0, \quad\left(1.19_{-0.17}^{+0.21}\right.$, $\left.0.91_{-0.55}^{+0.69}\right)$ at $f_{a 2}=0,\left(1.26_{-0.18}^{+0.20}, 0.53_{-0.50}^{+0.64}\right)$ at $f_{\Lambda 1}=0$, and $\left(1.24_{-0.17}^{+0.19}, 0.55_{-0.51}^{+0.64}\right)$ at $f_{\Lambda 1}^{Z \gamma}=0$. The values obtained for the different analyses vary because of the different categorization and observables in each $a_{i}$ analysis.

The combination of on-shell and off-shell regions allows the setting of tighter constraints on $f_{a i} \cos \left(\phi_{a i}\right)$ using the probability densities defined in Eqs. (9) and (10). As discussed above, the on-shell region is analyzed using the 2015, 2016, and 2017 data, and the earlier Run 1 data. The off-shell region is analyzed using only 2016 and 2017 data because no such analysis of the three anomalous couplings has been performed with the Run 1 or 2015 data in this region. The one-parameter likelihood scans of $f_{a i} \cos \left(\phi_{a i}\right)$ combining all such available on-shell and off-shell events is shown for two cases in Fig. 6, either with $\Gamma_{H}$ unconstrained in the fit or with the constraint $\Gamma_{H}=\Gamma_{H}^{S M}$. The corresponding $68 \%$ and $95 \%$ C.L. constraints are summarized in Table VI. The full two-parameter likelihood scans of $f_{a i} \cos \left(\phi_{a i}\right)$ and $\Gamma_{H}$ are likewise shown in Fig. 6. Using the transformation in Eq. (5), the $f_{a i} \cos \left(\phi_{a i}\right)$ results can be interpreted for the coupling parameters used in Eq. (2), as shown in Table VII.

Limits on $\Gamma_{H}$ are set by combining events from the onshell and off-shell regions. The left-hand panel of Fig. 7 shows the results of the likelihood scans of $\Gamma_{H}$ for the 2016 and 2017 period of the $13 \mathrm{TeV}$ run and for the combined data set from collisions at 7,8 and $13 \mathrm{TeV}$ under the assumption of SM-like couplings. The small contribution from the 2015 data set is not considered in this case, but the Run 1 analysis includes both the on-shell and off-shell regions in the analysis of the $H \rightarrow Z Z \rightarrow 4 \ell$ decay $[11,13]$. 

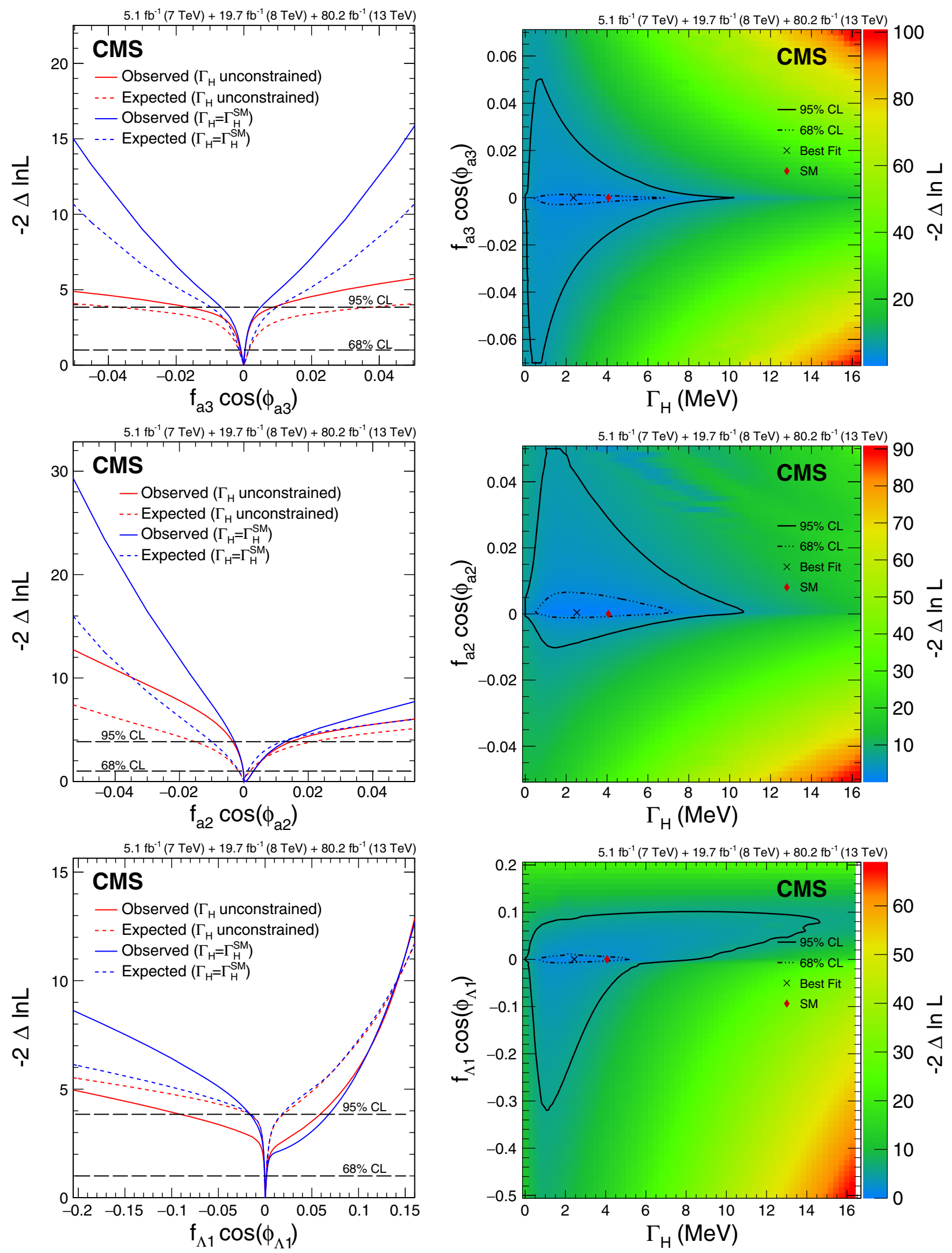

FIG. 6. Constraints on $f_{a 3} \cos \left(\phi_{a 3}\right)$ (top), $f_{a 2} \cos \left(\phi_{a 2}\right)$ (middle), and $f_{\Lambda 1} \cos \left(\phi_{\Lambda 1}\right)$ (bottom) from the combined Run 1 and Run 2 data set using both on-shell and off-shell events. Left plots: likelihood scans of the parameters of interest with unconstrained $\Gamma_{H}$ (red) or assuming $\Gamma_{H}=\Gamma_{H}^{\mathrm{SM}}$ (blue). The dashed horizontal lines show the $68 \%$ and 95\% C.L. regions. Right plots: observed two-parameter $\left(\Gamma_{H}, f_{a i} \cos \left(\phi_{a i}\right)\right)$ likelihood scans. The two-parameter $68 \%$ and $95 \%$ C.L. regions are indicated with the dashed and solid curves, respectively. 
TABLE VII. Summary of the allowed 95\% C.L. intervals for the anomalous $H V V$ couplings using results in Table VI. The coupling ratios are assumed to be real and include the factor $\cos \left(\phi_{\Lambda 1}\right)$ or $\cos \left(\phi_{\Lambda 1}^{Z \gamma}\right)= \pm 1$.

\begin{tabular}{lccc}
\hline \hline Parameter & Scenario & Observed & Expected \\
\hline$a_{3} / a_{1}$ & On-shell & {$[-1.13,0.80]$} & {$[-0.76,0.76]$} \\
& Any $\Gamma_{H}$ & {$[-0.33,0.24]$} & {$[-0.50,0.50]$} \\
& $\Gamma_{H}=\Gamma_{H}^{S M}$ & {$[-0.21,0.18]$} & {$[-0.25,0.25]$} \\
$a_{2} / a_{1}$ & On-shell & {$[-0.12,0.26]$} & {$[-0.24,0.31]$} \\
& Any $\Gamma_{H}$ & {$[-0.098,0.202]$} & {$[-0.21,0.25]$} \\
& $\Gamma_{H}=\Gamma_{H}^{S M}$ & {$[-0.089,0.189]$} & {$[-0.17,0.18]$} \\
$\left(\Lambda_{1} \sqrt{\left|a_{1}\right|}\right) \cos \left(\phi_{\Lambda 1}\right)(\mathrm{GeV})$ & On-shell & {$[-\infty,-130] \cup[160, \infty]$} & {$[-\infty,-180] \cup[210, \infty]$} \\
& Any $\Gamma_{H}$ & {$[-\infty,-160] \cup[180, \infty]$} & {$[-\infty,-250] \cup[240, \infty]$} \\
& $\Gamma_{H}=\Gamma_{H}^{S M}$ & {$[-\infty,-250] \cup[170, \infty]$} & {$[-\infty,-260] \cup[250, \infty]$} \\
$\left(\Lambda_{1}^{Z \gamma} \sqrt{\left|a_{1}\right|}\right) \cos \left(\phi_{\Lambda 1}^{Z Z_{\gamma}}\right)(\mathrm{GeV})$ & On-shell & {$[-\infty,-170] \cup[100, \infty]$} & {$[-\infty,-200] \cup[130, \infty]$} \\
\hline \hline
\end{tabular}

The combined results are listed in Table VIII. The best fit $\left(\mu_{F}, \mu_{V}\right)$ values in these results are $\left(1.20_{-0.16}^{+0.19}, 0.62_{-0.43}^{+0.57}\right)$ when $\Gamma_{H}=\Gamma_{H}^{\mathrm{SM}}$, and $\left(1.21_{-0.17}^{+0.19}, 0.65_{-0.45}^{+0.61}\right)$ when $\Gamma_{H}$ is unconstrained. The width constraints are also placed with the $f_{a 3} \cos \left(\phi_{a 3}\right), f_{a 2} \cos \left(\phi_{a 2}\right)$, or $f_{\Lambda 1} \cos \left(\phi_{\Lambda 1}\right)$ parameters unconstrained, and are shown in Fig. 7 right panel and summarized in Table IX. These results are obtained with the same fit configurations as for the study of anomalous couplings in the combination of the on-shell and off-shell regions.

The systematic uncertainties mostly cancel in the ratios of cross sections in the measurement of fractional parameters $f_{a i} \cos \left(\phi_{a i}\right)$, and are therefore negligible. The width constraints are also dominated by the statistical uncertainties, but because of the nontrivial dependence of systematic uncertainties on $m_{4 \ell}$, their dominant contributions may be worth examination. The two leading theoretical and two leading experimental uncertainties affecting the width constraints (observed and expected at $68 \%$ C.L.) are the uncertainty on the NLO EW corrections for the $q \bar{q} \rightarrow 4 \ell$ background ( \pm 0.5 and $\pm 1.9 \mathrm{MeV})$, the variation of renormalization scale in gluon fusion $( \pm 0.2$ and $\pm 0.4 \mathrm{MeV})$, the muon efficiency uncertainty $( \pm 0.1$ and $\pm 0.4 \mathrm{MeV})$, and the electron efficiency uncertainty $( \pm 0.1$ and $\pm 0.3 \mathrm{MeV})$.

The width constraints could also be reinterpreted as an off-shell signal strength with a change of parameters. For
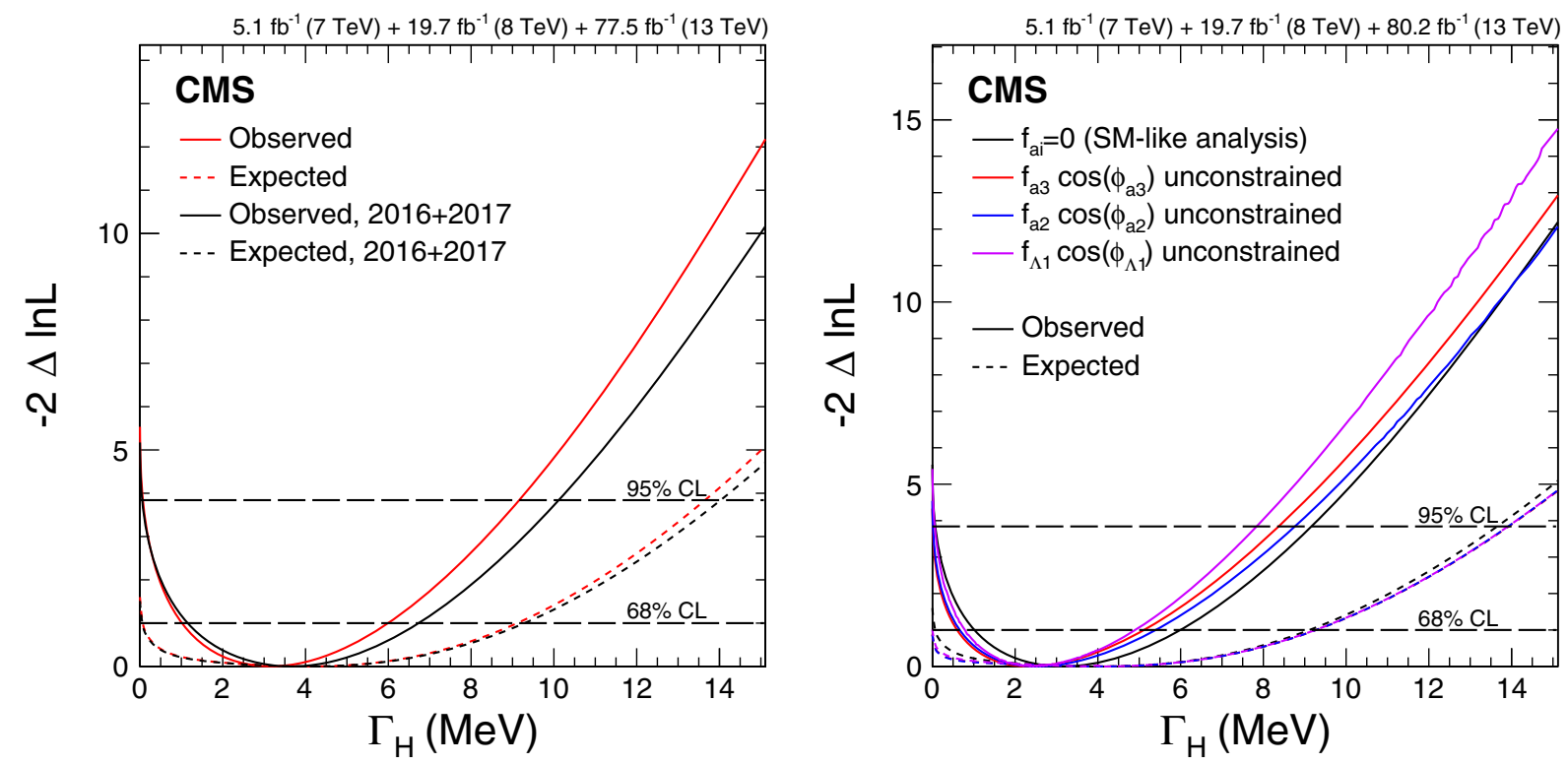

FIG. 7. Observed (solid) and expected (dashed) likelihood scans of $\Gamma_{H}$. Left plot: results of the SM-like couplings analysis are shown using the data only from 2016 and 2017 (black) or from the combination of Run 1 and Run 2 (red), which do not include 2015 data. Right plot: results of the combined Run 1 and Run 2 data analyses, with 2015 data included in the on-shell case, for the SM-like couplings or with three unconstrained anomalous coupling parameters, $f_{a 3} \cos \left(\phi_{a 3}\right)$ (red), $f_{a 2} \cos \left(\phi_{a 2}\right)$ (blue), and $f_{\Lambda 1} \cos \left(\phi_{\Lambda 1}\right)$ (violet). The dashed horizontal lines show the $68 \%$ and $95 \%$ C.L. regions. 
TABLE VIII. Summary of the total width $\Gamma_{H}$ measurement, showing the allowed $68 \%$ C.L. (central values with uncertainties) and $95 \%$ C.L. (in square brackets). The limits are reported for the SM-like couplings using the Run 1 and Run 2 combination.

\begin{tabular}{lcc}
\hline \hline Parameter & Observed & Expected \\
\hline$\Gamma_{H}(\mathrm{MeV})$ & $3.2_{-2.2}^{+2.8}[0.08,9.16]$ & $4.1_{-4.0}^{+5.0}[0.0,13.7]$ \\
\hline \hline
\end{tabular}

TABLE IX. Summary of the total width $\Gamma_{H}$ measurements, showing allowed $68 \%$ C.L. (central values with uncertainties) and 95\% C.L. (in square brackets). The $\Gamma_{H}$ limits are reported for the anomalous coupling parameter of interest unconstrained using the Run 1 and Run 2 combination.

\begin{tabular}{cccc}
\hline \hline Parameter & $\begin{array}{c}\text { Unconstrained } \\
\text { parameter }\end{array}$ & Observed & Expected \\
\hline$\Gamma_{H}$ & $f_{a 3} \cos \left(\phi_{a 3}\right)$ & $2.4_{-1.8}^{+2.7}[0.02$, & $4.1_{-4.1}^{+5.2}[0.0$, \\
$(\mathrm{MeV})$ & & $8.38]$ & $13.9]$ \\
$\Gamma_{H}$ & $f_{a 2} \cos \left(\phi_{a 2}\right)$ & $2.5_{-1.8}^{+2.9}[0.02$, & $4.1_{-4.1}^{+5.2}[0.0$, \\
$(\mathrm{MeV})$ & & $8.76]$ & $13.9]$ \\
$\Gamma_{H}$ & $f_{\Lambda 1} \cos \left(\phi_{\Lambda 1}\right)$ & $2.4_{-1.6}^{+2.5}[0.06$, & $4.1_{-4.1}^{+5.2}[0.0$, \\
$(\mathrm{MeV})$ & & $7.84]$ & $13.9]$ \\
\hline \hline
\end{tabular}

TABLE X. Summary of allowed $68 \%$ C.L. (central values with uncertainties) and $95 \%$ C.L. (in square brackets) intervals for $\mu^{\text {off-shell }}, \mu_{F}^{\text {off-shell }}$, and $\mu_{V}^{\text {off-shell }}$ obtained from the analysis of the combination of Run 1 and Run 2 off-shell data sets.

\begin{tabular}{lcc}
\hline \hline Parameter & Observed & Expected \\
\hline$\mu^{\text {off-shell }}$ & $0.78_{-0.53}^{+0.72}[0.02,2.28]$ & $1.00_{-0.99}^{+1.20}[0.0,3.2]$ \\
$\mu_{F}^{\text {off-shell }}$ & $0.86_{-0.68}^{+0.92}[0.0,2.7]$ & $1.0_{-1.0}^{+1.3}[0.0,3.5]$ \\
$\mu_{V}^{\text {off-shell }}$ & $0.67_{-0.61}^{+1.26}[0.0,3.6]$ & $1.0_{-1.0}^{+3.8}[0.0,8.4]$ \\
\hline \hline
\end{tabular}

this interpretation, we perform an SM-like analysis of only the off-shell events, where the signal strength is modified by the parameter $\mu^{\text {off-shell }}$ common to all production mechanisms in Eqs. (1) and (10), with $\Gamma_{H}=\Gamma_{0}=\Gamma_{H}^{\mathrm{SM}}$ and the SM expectation corresponding to $\mu^{\text {off-shell }}=1$. In addition, we also perform a fit of the off-shell events with two unconstrained parameters $\mu_{F}^{\text {off-shell }}$ and $\mu_{V}^{\text {off-shell }}$, which express the signal strengths in the gluon fusion and EW processes, respectively. These constraints are summarized in Table X.

\section{SUMMARY}

Studies of on-shell and off-shell $H$ boson production in the four-lepton final state are presented, using data from the CMS experiment at the LHC that correspond to an integrated luminosity of $80.2 \mathrm{fb}^{-1}$ at a center-of-mass energy of $13 \mathrm{TeV}$. Joint constraints are set on the $H$ boson total width and parameters that express its anomalous couplings to two electroweak vector bosons. These results are combined with those obtained from the data collected at center-of-mass energies of 7 and $8 \mathrm{TeV}$, corresponding to integrated luminosities of 5.1 and $19.7 \mathrm{fb}^{-1}$, respectively. Kinematic information from the decay particles and the associated jets are combined using matrix element techniques to identify the production mechanism and increase sensitivity to the $H$ boson couplings in both production and decay. The constraints on anomalous $H V V$ couplings are found to be consistent with the standard model expectation in both on-shell and off-shell regions, as presented in Tables VI and VII. Under the assumption of a coupling structure similar to that in the standard model, the $H$ boson width is constrained to be $3.2_{-2.2}^{+2.8} \mathrm{MeV}$ while the expected constraint based on simulation is $4.1_{-4.0}^{+5.0} \mathrm{MeV}$, as shown in Table VIII. The constraints on the width remain similar with the inclusion of the tested anomalous $H V V$ interactions and are summarized in Table IX. The width results are also interpreted in terms of the $H$ boson signal strength in the off-shell region in Table X. The observed off-shell signal strength, or equivalently a nonzero value of the width, is more than 2 standard deviations away from a background-only hypothesis, which provides a new direction to measure $H$ boson properties when more data are available.

\section{ACKNOWLEDGMENTS}

We thank Markus Schulze for optimizing the JHUGEN Monte Carlo simulation program and matrix element library for this analysis. We congratulate our colleagues in the CERN accelerator departments for the excellent performance of the LHC and thank the technical and administrative staffs at CERN and at other CMS institutes for their contributions to the success of the CMS effort. In addition, we gratefully acknowledge the computing centers and personnel of the Worldwide LHC Computing Grid for delivering so effectively the computing infrastructure essential to our analyses. We also acknowledge the Maryland Advanced Research Computing Center (MARCC) for providing computing resources essential for this analysis. Finally, we acknowledge the enduring support for the construction and operation of the LHC and the CMS detector provided by the following funding agencies: BMBWF and FWF (Austria); FNRS and FWO (Belgium); CNPq, CAPES, FAPERJ, FAPERGS, and FAPESP (Brazil); MES (Bulgaria); CERN; CAS, MoST, and NSFC (China); COLCIENCIAS (Colombia); MSES and CSF (Croatia); RPF (Cyprus); SENESCYT (Ecuador); MoER, ERC IUT, and ERDF (Estonia); Academy of Finland, MEC, and HIP (Finland); CEA and CNRS/ IN2P3 (France); BMBF, DFG, and HGF (Germany); GSRT (Greece); NKFIA (Hungary); DAE and DST (India); IPM (Iran); SFI (Ireland); INFN (Italy); MSIP and NRF (Republic of Korea); MES (Latvia); LAS 
(Lithuania); MOE and UM (Malaysia); BUAP, CINVESTAV, CONACYT, LNS, SEP, and UASLP-FAI (Mexico); MOS (Montenegro); MBIE (New Zealand); PAEC (Pakistan); MSHE and NSC (Poland); FCT (Portugal); JINR (Dubna); MON, RosAtom, RAS, RFBR, and NRC KI (Russia); MESTD (Serbia); SEIDI, CPAN, PCTI, and FEDER (Spain); MOSTR (Sri Lanka); Swiss Funding Agencies (Switzerland); MST (Taipei); ThEPCenter, IPST, STAR, and NSTDA (Thailand); TUBITAK and TAEK (Turkey); NASU and SFFR (Ukraine); STFC (United Kingdom); DOE and NSF (USA). Individuals have received support from the Marie-Curie program and the European Research Council and Horizon 2020 Grant, contract No. 675440 (European Union); the Leventis Foundation; the A. P. Sloan Foundation; the Alexander von Humboldt Foundation; the Belgian Federal Science Policy Office; the Fonds pour la Formation à la Recherche dans l'Industrie et dans l'Agriculture (FRIA-Belgium); the Agentschap voor Innovatie door Wetenschap en Technologie (IWTBelgium); the F. R. S.-FNRS and FWO (Belgium) under the "Excellence of Science-EOS"-be.h project n. 30820817; the Ministry of Education, Youth and Sports (MEYS) of the Czech Republic; the Lendület ("Momentum") Program and the János Bolyai Research
Scholarship of the Hungarian Academy of Sciences, the New National Excellence Program ÚNKP, the NKFIA research Grants No. 123842, No. 123959, No. 124845, No. 124850, and No. 125105 (Hungary); the Council of Science and Industrial Research, India; the HOMING PLUS program of the Foundation for Polish Science, cofinanced from European Union, Regional Development Fund, the Mobility Plus program of the Ministry of Science and Higher Education, the National Science Center (Poland), contracts Harmonia 2014/14/M/ ST2/00428, Opus 2014/13/B/ST2/02543, 2014/15/B/ST2/ 03998, and 2015/19/B/ST2/02861, Sonata-bis 2012/07/E/ ST2/01406; the National Priorities Research Program by Qatar National Research Fund; the Programa Estatal de Fomento de la Investigación Científica y Técnica de Excelencia María de Maeztu, grant MDM-2015-0509 and the Programa Severo Ochoa del Principado de Asturias; the Thalis and Aristeia programs cofinanced by EU-ESF and the Greek NSRF; the Rachadapisek Sompot Fund for Postdoctoral Fellowship, Chulalongkorn University and the Chulalongkorn Academic into Its 2nd Century Project Advancement Project (Thailand); the Welch Foundation, contract C-1845; and the Weston Havens Foundation (USA).
[1] S. L. Glashow, Partial-symmetries of weak interactions, Nucl. Phys. 22, 579 (1961).

[2] F. Englert and R. Brout, Broken Symmetry and the Mass of Gauge Vector Mesons, Phys. Rev. Lett. 13, 321 (1964).

[3] P. W. Higgs, Broken symmetries, massless particles and gauge fields, Phys. Lett. 12, 132 (1964).

[4] P. W. Higgs, Broken Symmetries and the Masses of Gauge Bosons, Phys. Rev. Lett. 13, 508 (1964).

[5] G. S. Guralnik, C. R. Hagen, and T. W. B. Kibble, Global Conservation Laws and Massless Particles, Phys. Rev. Lett. 13, 585 (1964).

[6] S. Weinberg, A Model of Leptons, Phys. Rev. Lett. 19, 1264 (1967).

[7] A. Salam, Weak and electromagnetic interactions, in Elementary Particle Physics: Relativistic Groups and Analyticity, edited by Nils Svartholm (Almqvist \& Wiksell, Stockholm, 1968), p. 367, proceedings of the eighth Nobel symposium.

[8] ATLAS Collaboration, Observation of a new particle in the search for the Standard Model Higgs boson with the ATLAS detector at the LHC, Phys. Lett. B 716, 1 (2012).

[9] CMS Collaboration, Observation of a new boson at a mass of $125 \mathrm{GeV}$ with the CMS experiment at the LHC, Phys. Lett. B 716, 30 (2012).

[10] CMS Collaboration, Observation of a new boson with mass near $125 \mathrm{GeV}$ in $p p$ collisions at $\sqrt{s}=7$ and $8 \mathrm{TeV}$, J. High Energy Phys. 06 (2013) 081.
[11] CMS Collaboration, Constraints on the Higgs boson width from off-shell production and decay to Z-boson pairs, Phys. Lett. B 736, 64 (2014).

[12] ATLAS Collaboration, Constraints on the off-shell Higgs boson signal strength in the high-mass $Z Z$ and $W W$ final states with the ATLAS detector, Eur. Phys. J. C 75, 335 (2015).

[13] CMS Collaboration, Limits on the Higgs boson lifetime and width from its decay to four charged leptons, Phys. Rev. D 92, 072010 (2015).

[14] CMS Collaboration, Search for Higgs boson off-shell production in proton-proton collisions at 7 and $8 \mathrm{TeV}$ and derivation of constraints on its total decay width, J. High Energy Phys. 09 (2016) 051.

[15] ATLAS Collaboration, Constraints on off-shell Higgs boson production and the Higgs boson total width in $Z Z \rightarrow 4 \ell$ and $Z Z \rightarrow 2 \ell 2 \nu$ final states with the ATLAS detector, Phys. Lett. B 786, 223 (2018).

[16] F. Caola and K. Melnikov, Constraining the Higgs boson width with $Z Z$ production at the LHC, Phys. Rev. D 88, 054024 (2013).

[17] N. Kauer and G. Passarino, Inadequacy of zero-width approximation for a light Higgs boson signal, J. High Energy Phys. 08 (2012) 116.

[18] J. M. Campbell, R. K. Ellis, and C. Williams, Bounding the Higgs width at the LHC using full analytic results for $g g \rightarrow e^{-} e^{+} \mu^{-} \mu^{+}$, J. High Energy Phys. 04 (2014) 060. 
[19] CMS Collaboration, Precise determination of the mass of the Higgs boson and tests of compatibility of its couplings with the standard model predictions using proton collisions at 7 and $8 \mathrm{TeV}$, Eur. Phys. J. C 75, 212 (2015).

[20] ATLAS Collaboration, Measurement of the Higgs boson mass from the $H \rightarrow \gamma \gamma$ and $H \rightarrow Z Z^{*} \rightarrow 4 \ell$ channels with the ATLAS detector using $25 \mathrm{fb}^{-1}$ of $p p$ collision data, Phys. Rev. D 90, 052004 (2014).

[21] CMS Collaboration, Measurements of properties of the Higgs boson decaying into the four-lepton final state in $p p$ collisions at $\sqrt{s}=13 \mathrm{TeV}, \mathrm{J}$. High Energy Phys. 11 (2017) 047.

[22] D. de Florian et al., Handbook of LHC Higgs cross sections: 4. Deciphering the nature of the Higgs sector, CERN Report No. CERN-2017-002-M, 2017, https://e-publishing.cern.ch/ index.php/CYRM/issue/view/32.

[23] CMS Collaboration, On the Mass and Spin-Parity of the Higgs Boson Candidate via Its Decays to $Z$ Boson Pairs, Phys. Rev. Lett. 110, 081803 (2013).

[24] CMS Collaboration, Measurement of the properties of a Higgs boson in the four-lepton final state, Phys. Rev. D 89, 092007 (2014).

[25] CMS Collaboration, Constraints on the spin-parity and anomalous $H V V$ couplings of the Higgs boson in proton collisions at 7 and 8 TeV, Phys. Rev. D 92, 012004 (2015).

[26] CMS Collaboration, Combined search for anomalous pseudoscalar $H V V$ couplings in $V H(H \rightarrow b \bar{b})$ production and $H \rightarrow V V$ decay, Phys. Lett. B 759, 672 (2016).

[27] CMS Collaboration, Constraints on anomalous Higgs boson couplings using production and decay information in the four-lepton final state, Phys. Lett. B 775, 1 (2017).

[28] ATLAS Collaboration, Evidence for the spin-0 nature of the Higgs boson using ATLAS data, Phys. Lett. B 726, 120 (2013).

[29] ATLAS Collaboration, Study of the spin and parity of the Higgs boson in diboson decays with the ATLAS detector, Eur. Phys. J. C 75, 476 (2015).

[30] ATLAS Collaboration, Test of $C P$ invariance in vectorboson fusion production of the Higgs boson using the optimal observable method in the ditau decay channel with the ATLAS detector, Eur. Phys. J. C 76, 658 (2016).

[31] ATLAS Collaboration, Measurement of inclusive and differential cross sections in the $H \rightarrow Z Z^{*} \rightarrow 4 \ell$ decay channel in $p p$ collisions at $\sqrt{s}=13 \mathrm{TeV}$ with the ATLAS detector, J. High Energy Phys. 10 (2017) 132.

[32] ATLAS Collaboration, Measurement of the Higgs boson coupling properties in the $H \rightarrow Z Z^{*} \rightarrow 4 \ell$ decay channel at $\sqrt{s}=13 \mathrm{TeV}$ with the ATLAS detector, J. High Energy Phys. 03 (2018) 095.

[33] ATLAS Collaboration, Measurements of Higgs boson properties in the diphoton decay channel with $36 \mathrm{fb}^{-1}$ of $p p$ collision data at $\sqrt{s}=13 \mathrm{TeV}$ with the ATLAS detector, Phys. Rev. D 98, 052005 (2018).

[34] J. S. Gainer, J. Lykken, K. T. Matchev, S. Mrenna, and M. Park, Beyond geolocating: Constraining higher dimensional operators in $H \rightarrow 4 \ell$ with off-shell production and more, Phys. Rev. D 91, 035011 (2015).

[35] C. Englert and M. Spannowsky, Limitations and opportunities of off-shell coupling measurements, Phys. Rev. D 90, 053003 (2014).
[36] M. Ghezzi, G. Passarino, and S. Uccirati, Bounding the Higgs width using effective field theory, Proc. Sci. LL2014 (2014) 072.

[37] CMS Collaboration, Search for a new scalar resonance decaying to a pair of $Z$ bosons in proton-proton collisions at $\sqrt{s}=13 \mathrm{TeV}$, J. High Energy Phys. 06 (2018) 127.

[38] C. A. Nelson, Correlation between decay planes in Higgsboson decays into a $W$ pair (into a $Z$ pair), Phys. Rev. D 37, 1220 (1988).

[39] A. Soni and R. M. Xu, Probing $C P$ violation via Higgs decays to four leptons, Phys. Rev. D 48, 5259 (1993).

[40] T. Plehn, D. L. Rainwater, and D. Zeppenfeld, Determining the Structure of Higgs Couplings at the LHC, Phys. Rev. Lett. 88, 051801 (2002).

[41] S. Y. Choi, D. J. Miller, M. M. Mühlleitner, and P. M. Zerwas, Identifying the Higgs spin and parity in decays to Z pairs, Phys. Lett. B 553, 61 (2003).

[42] C. P. Buszello, I. Fleck, P. Marquard, and J. J. van der Bij, Prospective analysis of spin- and $C P$-sensitive variables in $H \rightarrow Z Z \rightarrow \ell_{1}^{+} \ell_{1}^{-} \ell_{2}^{+} \ell_{2}^{-}$at the LHC, Eur. Phys. J. C 32, 209 (2004).

[43] V. Hankele, G. Klamke, D. Zeppenfeld, and T. Figy, Anomalous Higgs boson couplings in vector boson fusion at the CERN LHC, Phys. Rev. D 74, 095001 (2006).

[44] E. Accomando et al., 1st Workshop on CP studies and nonstandard Higgs physics, CERN Report No. CERN-2006009, 2006, http://cds.cern.ch/record/887410.

[45] R. M. Godbole, D. J. Miller, and M. Margarete Mühlleitner, Aspects of $C P$ violation in the $H Z Z$ coupling at the LHC, J. High Energy Phys. 12 (2007) 031.

[46] K. Hagiwara, Q. Li, and K. Mawatari, Jet angular correlation in vector-boson fusion processes at hadron colliders, J. High Energy Phys. 07 (2009) 101.

[47] Y. Gao, A. V. Gritsan, Z. Guo, K. Melnikov, M. Schulze, and Nhan V. Tran, Spin determination of single-produced resonances at hadron colliders, Phys. Rev. D 81, 075022 (2010).

[48] A. De Rújula, J. Lykken, M. Pierini, C. Rogan, and M. Spiropulu, Higgs look-alikes at the LHC, Phys. Rev. D 82, 013003 (2010).

[49] N. D. Christensen, T. Han, and Y. Li, Testing $C P$ violation in ZZH interactions at the LHC, Phys. Lett. B 693, 28 (2010).

[50] S. Bolognesi, Y. Gao, A. V. Gritsan, K. Melnikov, M. Schulze, N. V. Tran, and A. Whitbeck, Spin and parity of a single-produced resonance at the LHC, Phys. Rev. D 86, 095031 (2012).

[51] J. Ellis, D. S. Hwang, V. Sanz, and T. You, A fast track towards the "Higgs" spin and parity, J. High Energy Phys. 11 (2012) 134.

[52] Y. Chen, N. Tran, and R. Vega-Morales, Scrutinizing the Higgs signal and background in the $2 e 2 \mu$ golden channel, J. High Energy Phys. 01 (2013) 182.

[53] P. Artoisenet, P. de Aquino, F. Demartin, R. Frederix, S. Frixione, F. Maltoni, M. K. Mandal, P. Mathews, K. Mawatari, V. Ravindran, S. Seth, P. Torrielli, and M. Zaro, A framework for Higgs characterisation, J. High Energy Phys. 11 (2013) 043. 
[54] I. Anderson, S. Bolognesi, F. Caola, Y. Gao, A. V. Gritsan, C. B. Martin, K. Melnikov, M. Schulze, N. V. Tran, A. Whitbeck, and Y. Zhou, Constraining anomalous $H V V$ interactions at proton and lepton colliders, Phys. Rev. D 89, 035007 (2014).

[55] M. Chen, T. Cheng, J. S. Gainer, A. Korytov, K. T. Matchev, P. Milenovic, G. Mitselmakher, M. Park, A. Rinkevicius, and M. Snowball, Role of interference in unraveling the $Z Z$ couplings of the newly discovered boson at the LHC, Phys. Rev. D 89, 034002 (2014).

[56] M. J. Dolan, P. Harris, M. Jankowiak, and M. Spannowsky, Constraining $\mathrm{CP}$-violating Higgs sectors at the LHC using gluon fusion, Phys. Rev. D 90, 073008 (2014).

[57] M. Gonzalez-Alonso, A. Greljo, G. Isidori, and D. Marzocca, Pseudo-observables in Higgs decays, Eur. Phys. J. C 75, 128 (2015).

[58] A. Greljo, G. Isidori, J. M. Lindert, and D. Marzocca, Pseudo-observables in electroweak Higgs production, Eur. Phys. J. C 76, 158 (2016).

[59] A. V. Gritsan, R. Röntsch, M. Schulze, and M. Xiao, Constraining anomalous Higgs boson couplings to the heavy flavor fermions using matrix element techniques, Phys. Rev. D 94, 055023 (2016).

[60] CMS Collaboration, The CMS experiment at the CERN LHC, J. Instrum. 3, S08004 (2008).

[61] S. Frixione, P. Nason, and C. Oleari, Matching NLO QCD computations with parton shower simulations: The POWHEG method, J. High Energy Phys. 11 (2007) 070.

[62] E. Bagnaschi, G. Degrassi, P. Slavich, and A. Vicini, Higgs production via gluon fusion in the POWHEG approach in the SM and in the MSSM, J. High Energy Phys. 02 (2012) 088.

[63] P. Nason and C. Oleari, NLO Higgs boson production via vector-boson fusion matched with shower in POWHEG, J. High Energy Phys. 02 (2010) 037.

[64] G. Luisoni, P. Nason, C. Oleari, and F. Tramontano, $H W^{ \pm} / H Z+0$ and 1 jet at NLO with the POWHEG BOX interfaced to GoSam and their merging within MiNLO, J. High Energy Phys. 10 (2013) 083.

[65] H. B. Hartanto, B. Jager, L. Reina, and D. Wackeroth, Higgs boson production in association with top quarks in the POWHEG BOX, Phys. Rev. D 91, 094003 (2015).

[66] K. Hamilton, P. Nason, and G. Zanderighi, MINLO: Multiscale improved NLO, J. High Energy Phys. 10 (2012) 155.

[67] J. M. Campbell and R. K. Ellis, MCFM for the Tevatron and the LHC, Nucl. Phys. B, Proc. Suppl. 205-206, 10 (2010).

[68] J. M. Campbell, R. K. Ellis, and C. Williams, Vector boson pair production at the LHC, J. High Energy Phys. 07 (2011) 018.

[69] J. M. Campbell and R. K. Ellis, Higgs constraints from vector boson fusion and scattering, J. High Energy Phys. 04 (2015) 030.

[70] A. Ballestrero, A. Belhouari, G. Bevilacqua, V. Kashkan, and E. Maina, PHANTOM: A Monte Carlo event generator for six parton final states at high energy colliders, Comput. Phys. Commun. 180, 401 (2009).

[71] S. Catani and M. Grazzini, Next-to-Next-to-Leading-Order Subtraction Formalism in Hadron Collisions and Its Application to Higgs Boson Production at the Large Hadron Collider, Phys. Rev. Lett. 98, 222002 (2007).
[72] M. Grazzini, NNLO predictions for the Higgs boson signal in the $H \rightarrow W W \rightarrow \ell \nu \ell \nu$ and $H \rightarrow Z Z \rightarrow 4 \ell$ decay channels, J. High Energy Phys. 02 (2008) 043.

[73] M. Grazzini and H. Sargsyan, Heavy-quark mass effects in Higgs boson production at the LHC, J. High Energy Phys. 09 (2013) 129.

[74] F. Caola, K. Melnikov, R. Röntsch, and L. Tancredi, QCD corrections to $\mathrm{ZZ}$ production in gluon fusion at the LHC, Phys. Rev. D 92, 094028 (2015).

[75] K. Melnikov and M. Dowling, Production of two Z-bosons in gluon fusion in the heavy top quark approximation, Phys. Lett. B 744, 43 (2015).

[76] J. M. Campbell, R. K. Ellis, M. Czakon, and S. Kirchner, Two loop correction to interference in $g g \rightarrow Z Z$, J. High Energy Phys. 08 (2016) 011.

[77] F. Caola, M. Dowling, K. Melnikov, R. Röntsch, and L. Tancredi, QCD corrections to vector boson pair production in gluon fusion including interference effects with off-shell Higgs at the LHC, J. High Energy Phys. 07 (2016) 087.

[78] M. Grazzini, S. Kallweit, and D. Rathlev, ZZ production at the LHC: Fiducial cross sections and distributions in NNLO QCD, Phys. Lett. B 750, 407 (2015).

[79] S. Gieseke, T. Kasprzik, and J. H. Kuehn, Vector-boson pair production and electroweak corrections in HERWIG++, Eur. Phys. J. C 74, 2988 (2014).

[80] J. Baglio, L. D. Ninh, and M. M. Weber, Massive gauge boson pair production at the LHC: A next-to-leading order story, Phys. Rev. D 88, 113005 (2013); 94, 099902 (E) (2016).

[81] R. D. Ball, V. Bertone, F. Cerutti, L. Del Debbio, S. Forte, A. Guffanti, J. I. Latorre, J. Rojo, and M. Ubiali (NNPDF), Unbiased global determination of parton distributions and their uncertainties at NNLO and at LO, Nucl. Phys. B855, 153 (2012).

[82] T. Sjöstrand, S. Ask, J. R. Christiansen, R. Corke, N. Desai, P. Ilten, S. Mrenna, S. Prestel, C. O. Rasmussen, and P.Z. Skands, An introduction to PYTHIA 8.2, Comput. Phys. Commun. 191, 159 (2015).

[83] S. Agostinelli et al. (GEANT4 Collaboration), GEANT4-a simulation toolkit, Nucl. Instrum. Methods Phys. Res., Sect. A 506, 250 (2003).

[84] CMS Collaboration, Particle-flow reconstruction and global event description with the CMS detector, J. Instrum. 12, P10003 (2017).

[85] M. Cacciari, G. P. Salam, and G. Soyez, The anti- $k_{T}$ jet clustering algorithm, J. High Energy Phys. 04 (2008) 063.

[86] M. Cacciari, G. P. Salam, and G. Soyez, FastJet user manual, Eur. Phys. J. C 72, 1896 (2012).

[87] CMS Collaboration, Identification of $b$-quark jets with the CMS experiment, J. Instrum. 8, P04013 (2013).

[88] CMS Collaboration, Identification of heavy-flavour jets with the CMS detector in $p p$ collisions at $13 \mathrm{TeV}, \mathrm{J}$. Instrum. 13, P05011 (2018).

[89] R. J. Barlow, Extended maximum likelihood, Nucl. Instrum. Methods Phys. Res., Sect. A 297, 496 (1990).

[90] M. Oreglia, A study of the reactions $\psi^{\prime} \rightarrow \gamma \gamma \psi$, Ph.D. thesis, Stanford University, 1980, SLAC Report No. SLAC-R-236, http://www.slac.stanford.edu/cgi-wrap/getdoc/slac-r-236 .pdf. 
[91] W. Verkerke and D. P. Kirkby, The RooFit toolkit for data modeling, arXiv:physics/0306116.

[92] R. Brun and F. Rademakers, ROOT: An object oriented data analysis framework, Nucl. Instrum. Methods Phys. Res., Sect. A 389, 81 (1997).

[93] S. S. Wilks, The large-sample distribution of the likelihood ratio for testing composite hypotheses, Ann. Math. Stat. 9, 60 (1938).
[94] CMS Collaboration, CMS luminosity measurements for the 2016 data taking period, CMS Physics Analysis Summary Report No. CMS-PAS-LUM-17-001, 2017, https://cds.cern .ch/record/2257069.

[95] CMS Collaboration, CMS luminosity measurement for the 2017 data taking period at $\sqrt{s}=13 \mathrm{TeV}$, CMS Physics Analysis Summary Report No. CMS-PAS-LUM-17-004, 2018, https://cds.cern.ch/record/2621960.

A. M. Sirunyan, ${ }^{1}$ A. Tumasyan, ${ }^{1}$ W. Adam, ${ }^{2}$ F. Ambrogi, ${ }^{2}$ E. Asilar, ${ }^{2}$ T. Bergauer, ${ }^{2}$ J. Brandstetter, ${ }^{2}$ M. Dragicevic, ${ }^{2}$ J. Erö, ${ }^{2}$ A. Escalante Del Valle, ${ }^{2}$ M. Flechl, ${ }^{2}$ R. Frühwirth, ${ }^{2, b}$ V. M. Ghete, ${ }^{2}$ J. Hrubec, ${ }^{2}$ M. Jeitler, ${ }^{2, b}$ N. Krammer, ${ }^{2}$ I. Krätschmer, ${ }^{2}$ D. Liko, ${ }^{2}$ T. Madlener, ${ }^{2}$ I. Mikulec, ${ }^{2}$ N. Rad, ${ }^{2}$ H. Rohringer, ${ }^{2}$ J. Schieck ${ }^{2, b}$ R. Schöfbeck, ${ }^{2}$ M. Spanring, ${ }^{2}$ D. Spitzbart, ${ }^{2}$ W. Waltenberger, ${ }^{2}$ J. Wittmann, ${ }^{2}$ C.-E. Wulz, ${ }^{2, b}$ M. Zarucki, ${ }^{2}$ V. Chekhovsky, ${ }^{3}$ V. Mossolov, ${ }^{3}$ J. Suarez Gonzalez, ${ }^{3}$ E. A. De Wolf, ${ }^{4}$ D. Di Croce, ${ }^{4}$ X. Janssen, ${ }^{4}$ J. Lauwers, ${ }^{4}$ A. Lelek, ${ }^{4}$ M. Pieters, ${ }^{4}$ H. Van Haevermaet,${ }^{4}$ P. Van Mechelen, ${ }^{4}$ N. Van Remortel, ${ }^{4}$ S. Abu Zeid, ${ }^{5}$ F. Blekman, ${ }^{5}$ J. D'Hondt, ${ }^{5}$ J. De Clercq, ${ }^{5}$ K. Deroover, ${ }^{5}$ G. Flouris, ${ }^{5}$ D. Lontkovskyi, ${ }^{5}$ S. Lowette, ${ }^{5}$ I. Marchesini, ${ }^{5}$ S. Moortgat, ${ }^{5}$ L. Moreels, ${ }^{5}$ Q. Python, ${ }^{5}$ K. Skovpen, ${ }^{5}$ S. Tavernier, ${ }^{5}$ W. Van Doninck, ${ }^{5}$ P. Van Mulders, ${ }^{5}$ I. Van Parijs, ${ }^{5}$ D. Beghin, ${ }^{6}$ B. Bilin, ${ }^{6}$ H. Brun, ${ }^{6}$ B. Clerbaux, ${ }^{6}$ G. De Lentdecker, ${ }^{6}$ H. Delannoy, ${ }^{6}$ B. Dorney, ${ }^{6}$ G. Fasanella, ${ }^{6}$ L. Favart, ${ }^{6}$ A. Grebenyuk, ${ }^{6}$ A. K. Kalsi, ${ }^{6}$ T. Lenzi, ${ }^{6}$ J. Luetic, ${ }^{6}$ N. Postiau, ${ }^{6}$ E. Starling, ${ }^{6}$ L. Thomas, ${ }^{6}$ C. Vander Velde, ${ }^{6}$ P. Vanlaer, ${ }^{6}$ D. Vannerom, ${ }^{6}$ Q. Wang, ${ }^{6}$ T. Cornelis, ${ }^{7}$ D. Dobur, ${ }^{7}$ A. Fagot, ${ }^{7}$ M. Gul, ${ }^{7}$ I. Khvastunov, ${ }^{7, c}$ D. Poyraz, ${ }^{7}$ C. Roskas, ${ }^{7}$ D. Trocino, ${ }^{7}$ M. Tytgat, ${ }^{7}$ W. Verbeke, ${ }^{7}$ B. Vermassen, ${ }^{7}$ M. Vit, ${ }^{7}$ N. Zaganidis, ${ }^{7}$ H. Bakhshiansohi, ${ }^{8}$ O. Bondu, ${ }^{8}$ G. Bruno, ${ }^{8}$ C. Caputo, ${ }^{8}$ P. David, ${ }^{8}$ C. Delaere,${ }^{8}$ M. Delcourt, ${ }^{8}$ A. Giammanco, ${ }^{8}$ G. Krintiras, ${ }^{8}$

V. Lemaitre, ${ }^{8}$ A. Magitteri, ${ }^{8}$ K. Piotrzkowski, ${ }^{8}$ A. Saggio, ${ }^{8}$ M. Vidal Marono, ${ }^{8}$ P. Vischia, ${ }^{8}$ J. Zobec, ${ }^{8}$ F. L. Alves, ${ }^{9}$ G. A. Alves, ${ }^{9}$ G. Correia Silva, ${ }^{9}$ C. Hensel, ${ }^{9}$ A. Moraes, ${ }^{9}$ M. E. Pol,,${ }^{9}$ P. Rebello Teles, ${ }^{9}$ E. Belchior Batista Das Chagas, ${ }^{10}$ W. Carvalho, ${ }^{10}$ J. Chinellato, ${ }^{10, \mathrm{~d}}$ E. Coelho, ${ }^{10}$ E. M. Da Costa, ${ }^{10}$ G. G. Da Silveira, ${ }^{10, \mathrm{e}}$ D. De Jesus Damiao, ${ }^{10}$ C. De Oliveira Martins, ${ }^{10}$ S. Fonseca De Souza,${ }^{10}$ H. Malbouisson, ${ }^{10}$ D. Matos Figueiredo, ${ }^{10}$ M. Melo De Almeida,${ }^{10}$ C. Mora Herrera, ${ }^{10}$ L. Mundim, ${ }^{10}$ H. Nogima, ${ }^{10}$ W. L. Prado Da Silva, ${ }^{10}$ L. J. Sanchez Rosas, ${ }^{10}$ A. Santoro, ${ }^{10}$ A. Sznajder, ${ }^{10}$ M. Thiel,${ }^{10}$ E. J. Tonelli Manganote,${ }^{10, \mathrm{~d}}$ F. Torres Da Silva De Araujo, ${ }^{10}$ A. Vilela Pereira, ${ }^{10}$ S. Ahuja,${ }^{11 a}$ C. A. Bernardes, ${ }^{11 a}$

L. Calligaris, ${ }^{11 \mathrm{a}}$ T. R. Fernandez Perez Tomei, ${ }^{11 \mathrm{a}}$ E. M. Gregores, ${ }^{11 \mathrm{a}, 1 \mathrm{~b}}$ P. G. Mercadante, ${ }^{11 \mathrm{a}, 11 \mathrm{~b}}$ S. F. Novaes, ${ }^{1 \mathrm{a}}$

Sandra S. Padula, ${ }^{11 a}$ A. Aleksandrov, ${ }^{12}$ R. Hadjiiska, ${ }^{12}$ P. Iaydjiev, ${ }^{12}$ A. Marinov, ${ }^{12}$ M. Misheva, ${ }^{12}$ M. Rodozov, ${ }^{12}$ M. Shopova, ${ }^{12}$ G. Sultanov, ${ }^{12}$ A. Dimitrov, ${ }^{13}$ L. Litov ${ }^{13}$ B. Pavlov, ${ }^{13}$ P. Petkov,${ }^{13}$ W. Fang, ${ }^{14, f}$ X. Gao, ${ }^{14, f}$ L. Yuan, ${ }^{14}$ M. Ahmad, ${ }^{15}$ J. G. Bian, ${ }^{15}$ G. M. Chen, ${ }^{15}$ H. S. Chen,${ }^{15}$ M. Chen, ${ }^{15}$ Y. Chen,${ }^{15}$ C. H. Jiang, ${ }^{15}$ D. Leggat,${ }^{15}$ H. Liao, ${ }^{15}$ Z. Liu, ${ }^{15}$ S. M. Shaheen, ${ }^{15, \mathrm{~g}}$ A. Spiezia, ${ }^{15}$ J. Tao, ${ }^{15}$ E. Yazgan, ${ }^{15}$ H. Zhang, ${ }^{15}$ S. Zhang, ${ }^{15, g}$ J. Zhao, ${ }^{15}$ Y. Ban, ${ }^{16}$ G. Chen, ${ }^{16}$ A. Levin, ${ }^{16}$ J. Li, ${ }^{16}$ L. Li, ${ }^{16}$ Q. Li, ${ }^{16}$ Y. Mao, ${ }^{16}$ S. J. Qian, ${ }^{16}$ D. Wang, ${ }^{16}$ Y. Wang, ${ }^{17}$ C. Avila, ${ }^{18}$ A. Cabrera, ${ }^{18}$ C. A. Carrillo Montoya, ${ }^{18}$ L. F. Chaparro Sierra, ${ }^{18}$ C. Florez, ${ }^{18}$ C. F. González Hernández, ${ }^{18}$ M. A. Segura Delgado, ${ }^{18}$ B. Courbon, ${ }^{19}$ N. Godinovic, ${ }^{19}$ D. Lelas, ${ }^{19}$ I. Puljak, ${ }^{19}$ T. Sculac, ${ }^{19}$ Z. Antunovic, ${ }^{20}$ M. Kovac, ${ }^{20}$ V. Brigljevic, ${ }^{21}$ D. Ferencek, ${ }^{21}$ K. Kadija, ${ }^{21}$ B. Mesic, ${ }^{21}$ M. Roguljic, ${ }^{21}$ A. Starodumov, ${ }^{21, h}$ T. Susa, ${ }^{21}$ M. W. Ather, ${ }^{22}$ A. Attikis, ${ }^{22}$ M. Kolosova, ${ }^{22}$ G. Mavromanolakis, ${ }^{22}$ J. Mousa, ${ }^{22}$ C. Nicolaou, ${ }^{22}$ F. Ptochos,${ }^{22}$ P. A. Razis, ${ }^{22}$ H. Rykaczewski, ${ }^{22}$ M. Finger, ${ }^{23, i}$ M. Finger Jr., ${ }^{23, i}$ E. Ayala, ${ }^{24}$ E. Carrera Jarrin, ${ }^{25}$ A. A. Abdelalim, ${ }^{26, j, k}$ Y. Assran, ${ }^{26,1, \mathrm{~m}}$ A. Mohamed, ${ }^{26, \mathrm{k}}$ S. Bhowmik, ${ }^{27}$ A. Carvalho Antunes De Oliveira, ${ }^{27}$

R. K. Dewanjee, ${ }^{27}$ K. Ehataht, ${ }^{27}$ M. Kadastik, ${ }^{27}$ M. Raidal, ${ }^{27}$ C. Veelken, ${ }^{27}$ P. Eerola,${ }^{28}$ H. Kirschenmann, ${ }^{28}$ J. Pekkanen, ${ }^{28}$ M. Voutilainen, ${ }^{28}$ J. Havukainen, ${ }^{29}$ J. K. Heikkilä, ${ }^{29}$ T. Järvinen, ${ }^{29}$ V. Karimäki, ${ }^{29}$ R. Kinnunen, ${ }^{29}$ T. Lampén, ${ }^{29}$ K. Lassila-Perini, ${ }^{29}$ S. Laurila, ${ }^{29}$ S. Lehti, ${ }^{29}$ T. Lindén, ${ }^{29}$ P. Luukka, ${ }^{29}$ T. Mäenpää, ${ }^{29}$ H. Siikonen, ${ }^{29}$ E. Tuominen, ${ }^{29}$ J. Tuominiemi, ${ }^{29}$ T. Tuuva, ${ }^{30}$ M. Besancon, ${ }^{31}$ F. Couderc, ${ }^{31}$ M. Dejardin, ${ }^{31}$ D. Denegri, ${ }^{31}$ J. L. Faure, ${ }^{31}$ F. Ferri, ${ }^{31}$ S. Ganjour, ${ }^{31}$ A. Givernaud, ${ }^{31}$ P. Gras,${ }^{31}$ G. Hamel de Monchenault, ${ }^{31}$ P. Jarry,${ }^{31}$ C. Leloup,${ }^{31}$ E. Locci,${ }^{31}$ J. Malcles, ${ }^{31}$ G. Negro, ${ }^{31}$ J. Rander, ${ }^{31}$ A. Rosowsky, ${ }^{31}$ M. Ö. Sahin, ${ }^{31}$ M. Titov, ${ }^{31}$ A. Abdulsalam, ${ }^{32, n}$ C. Amendola, ${ }^{32}$ I. Antropov, ${ }^{32}$ F. Beaudette, ${ }^{32}$ P. Busson, ${ }^{32}$ C. Charlot,${ }^{32}$ R. Granier de Cassagnac, ${ }^{32}$ I. Kucher, ${ }^{32}$ A. Lobanov, ${ }^{32}$ J. Martin Blanco, ${ }^{32}$ C. Martin Perez, ${ }^{32}$ M. Nguyen, ${ }^{32}$ C. Ochando, ${ }^{32}$ G. Ortona, ${ }^{32}$ P. Paganini, ${ }^{32}$ J. Rembser, ${ }^{32}$ R. Salerno, ${ }^{32}$ J. B. Sauvan, ${ }^{32}$ Y. Sirois, ${ }^{32}$ A. G. Stahl Leiton, ${ }^{32}$ A. Zabi, ${ }^{32}$ A. Zghiche, ${ }^{32}$ J.-L. Agram, ${ }^{33,0}$ J. Andrea, ${ }^{33}$ D. Bloch, ${ }^{33}$ G. Bourgatte, ${ }^{33}$ J.-M. Brom, ${ }^{33}$ E. C. Chabert, ${ }^{33}$ V. Cherepanov, ${ }^{33}$ C. Collard, ${ }^{33}$ E. Conte, ${ }^{33,0}$ J.-C. Fontaine, ${ }^{33,0}$ D. Gelé, ${ }^{33}$ U. Goerlach, ${ }^{33}$ 
M. Jansová, ${ }^{33}$ A.-C. Le Bihan, ${ }^{33}$ N. Tonon, ${ }^{33}$ P. Van Hove, ${ }^{33}$ S. Gadrat, ${ }^{34}$ S. Beauceron, ${ }^{35}$ C. Bernet,${ }^{35}$ G. Boudoul, ${ }^{35}$ N. Chanon, ${ }^{35}$ R. Chierici, ${ }^{35}$ D. Contardo, ${ }^{35}$ P. Depasse, ${ }^{35}$ H. El Mamouni, ${ }^{35}$ J. Fay, ${ }^{35}$ L. Finco, ${ }^{35}$ S. Gascon, ${ }^{35}$ M. Gouzevitch, ${ }^{35}$ G. Grenier, ${ }^{35}$ B. Ille, ${ }^{35}$ F. Lagarde, ${ }^{35}$ I. B. Laktineh, ${ }^{35}$ H. Lattaud,${ }^{35}$ M. Lethuillier, ${ }^{35}$ L. Mirabito, ${ }^{35}$ S. Perries, ${ }^{35}$ A. Popov, ${ }^{35, p}$ V. Sordini, ${ }^{35}$ G. Touquet, ${ }^{35}$ M. Vander Donckt,${ }^{35}$ S. Viret, ${ }^{35}$ A. Khvedelidze, ${ }^{36, i}$ Z. Tsamalaidze,${ }^{37, i}$ C. Autermann, ${ }^{38}$ L. Feld ${ }^{38}$ M. K. Kiesel,${ }^{38}$ K. Klein, ${ }^{38}$ M. Lipinski, ${ }^{38}$ M. Preuten, ${ }^{38}$ M. P. Rauch, ${ }^{38}$ C. Schomakers, ${ }^{38}$ J. Schulz, ${ }^{38}$ M. Teroerde, ${ }^{38}$ B. Wittmer, ${ }^{38}$ A. Albert, ${ }^{39}$ M. Erdmann, ${ }^{39}$ S. Erdweg, ${ }^{39}$ T. Esch, ${ }^{39}$ R. Fischer, ${ }^{39}$ S. Ghosh, ${ }^{39}$ T. Hebbeker, ${ }^{39}$ C. Heidemann, ${ }^{39}$ K. Hoepfner, ${ }^{39}$ H. Keller, ${ }^{39}$ L. Mastrolorenzo, ${ }^{39}$ M. Merschmeyer, ${ }^{39}$ A. Meyer, ${ }^{39}$ P. Millet,${ }^{39}$ S. Mukherjee, ${ }^{39}$ T. Pook, ${ }^{39}$ A. Pozdnyakov, ${ }^{39}$ M. Radziej, ${ }^{39}$ H. Reithler, ${ }^{39}$ M. Rieger, ${ }^{39}$ A. Schmidt, ${ }^{39}$ D. Teyssier, ${ }^{39}$ S. Thüer, ${ }^{39}$ G. Flügge, ${ }^{40}$ O. Hlushchenko, ${ }^{40}$ T. Kress, ${ }^{40}$ T. Müller, ${ }^{40}$ A. Nehrkorn,${ }^{40}$ A. Nowack, ${ }^{40}$ C. Pistone,${ }^{40}$ O. Pooth,${ }^{40}$ D. Roy, ${ }^{40}$ H. Sert, ${ }^{40}$ A. Stahl,${ }^{40, q}$ M. Aldaya Martin, ${ }^{41}$ T. Arndt, ${ }^{41}$ C. Asawatangtrakuldee, ${ }^{41}$ I. Babounikau, ${ }^{41}$ K. Beernaert, ${ }^{41}$ O. Behnke, ${ }^{41}$ U. Behrens, ${ }^{41}$ A. Bermúdez Martínez, ${ }^{41}$ D. Bertsche, ${ }^{41}$ A. A. Bin Anuar, ${ }^{41}$ K. Borras, ${ }^{41, r}$ V. Botta, ${ }^{41}$ A. Campbell, ${ }^{41}$ P. Connor, ${ }^{41}$ C. Contreras-Campana, ${ }^{41}$ V. Danilov, ${ }^{41}$ A. De Wit, ${ }^{41}$ M. M. Defranchis, ${ }^{41}$ C. Diez Pardos, ${ }^{41}$ D. Domínguez Damiani, ${ }^{41}$ G. Eckerlin, ${ }^{41}$ T. Eichhorn, ${ }^{41}$ A. Elwood, ${ }^{41}$ E. Eren, ${ }^{41}$ E. Gallo, ${ }^{41, s}$ A. Geiser, ${ }^{41}$ J. M. Grados Luyando, ${ }^{41}$ A. Grohsjean, ${ }^{41}$ M. Guthoff, ${ }^{41}$ M. Haranko, ${ }^{41}$ A. Harb,${ }^{41}$ H. Jung, ${ }^{41}$ M. Kasemann, ${ }^{41}$ J. Keaveney, ${ }^{41}$ C. Kleinwort, ${ }^{41}$ J. Knolle, ${ }^{41}$ D. Krücker, ${ }^{41}$ W. Lange, ${ }^{41}$ T. Lenz, ${ }^{41}$ J. Leonard ${ }^{41}$ K. Lipka, ${ }^{41}$ W. Lohmann, ${ }^{41, t}$ R. Mankel, ${ }^{41}$ I.-A. Melzer-Pellmann, ${ }^{41}$ A. B. Meyer ${ }^{41}$ M. Meyer ${ }^{41}$ M. Missiroli, ${ }^{41}$ G. Mittag, ${ }^{41}$ J. Mnich ${ }^{41}$ V. Myronenko, ${ }^{41}$

S. K. Pflitsch, ${ }^{41}$ D. Pitzl, ${ }^{41}$ A. Raspereza, ${ }^{41}$ A. Saibel, ${ }^{41}$ M. Savitskyi, ${ }^{41}$ P. Saxena, ${ }^{41}$ P. Schütze,${ }^{41}$ C. Schwanenberger,${ }^{41}$ R. Shevchenko, ${ }^{41}$ A. Singh, ${ }^{41}$ H. Tholen, ${ }^{41}$ O. Turkot ${ }^{41}$ A. Vagnerini, ${ }^{41}$ M. Van De Klundert, ${ }^{41}$ G. P. Van Onsem, ${ }^{41}$ R. Walsh, ${ }^{41}$ Y. Wen, ${ }^{41}$ K. Wichmann, ${ }^{41}$ C. Wissing, ${ }^{41}$ O. Zenaiev, ${ }^{41}$ R. Aggleton, ${ }^{42}$ S. Bein, ${ }^{42}$ L. Benato, ${ }^{42}$ A. Benecke, ${ }^{42}$ T. Dreyer, ${ }^{42}$ A. Ebrahimi, ${ }^{42}$ E. Garutti ${ }^{42}$ D. Gonzalez, ${ }^{42}$ P. Gunnellini, ${ }^{42}$ J. Haller, ${ }^{42}$ A. Hinzmann, ${ }^{42}$ A. Karavdina, ${ }^{42}$ G. Kasieczka, ${ }^{42}$ R. Klanner, ${ }^{42}$ R. Kogler, ${ }^{42}$ N. Kovalchuk, ${ }^{42}$ S. Kurz, ${ }^{42}$ V. Kutzner, ${ }^{42}$ J. Lange, ${ }^{42}$ D. Marconi, ${ }^{42}$ J. Multhaup, ${ }^{42}$ M. Niedziela, ${ }^{42}$ C. E. N. Niemeyer, ${ }^{42}$ D. Nowatschin, ${ }^{42}$ A. Perieanu, ${ }^{42}$ A. Reimers, ${ }^{42}$ O. Rieger, ${ }^{42}$ C. Scharf, ${ }^{42}$ P. Schleper,${ }^{42}$ S. Schumann, ${ }^{42}$ J. Schwandt, ${ }^{42}$ J. Sonneveld, ${ }^{42}$ H. Stadie, ${ }^{42}$ G. Steinbrück,${ }^{42}$ F. M. Stober, ${ }^{42}$ M. Stöver, ${ }^{42}$ B. Vormwald ${ }^{42}$ I. Zoi ${ }^{42}$ M. Akbiyik, ${ }^{43}$ C. Barth, ${ }^{43}$ M. Baselga, ${ }^{43}$ S. Baur, ${ }^{43}$ E. Butz, ${ }^{43}$ R. Caspart, ${ }^{43}$ T. Chwalek, ${ }^{43}$ F. Colombo, ${ }^{43}$ W. De Boer, ${ }^{43}$ A. Dierlamm, ${ }^{43}$ K. El Morabit, ${ }^{43}$ N. Faltermann, ${ }^{43}$ B. Freund, ${ }^{43}$ M. Giffels, ${ }^{43}$ M. A. Harrendorf, ${ }^{43}$ F. Hartmann, ${ }^{43, q}$ S. M. Heindl, ${ }^{43}$ U. Husemann, ${ }^{43}$ I. Katkov, ${ }^{43, p}$ S. Kudella, ${ }^{43}$ S. Mitra, ${ }^{43}$ M. U. Mozer, ${ }^{43}$ Th. Müller, ${ }^{43}$ M. Musich, ${ }^{43}$ M. Plagge, ${ }^{43}$ G. Quast ${ }^{43}$ K. Rabbertz,${ }^{43}$ M. Schröder, ${ }^{43}$ I. Shvetsov, ${ }^{43}$ H. J. Simonis, ${ }^{43}$ R. Ulrich, ${ }^{43}$ S. Wayand, ${ }^{43}$ M. Weber, ${ }^{43}$ T. Weiler, ${ }^{43}$ C. Wöhrmann, ${ }^{43}$ R. Wolf, ${ }^{43}$ G. Anagnostou, ${ }^{44}$ G. Daskalakis, ${ }^{44}$ T. Geralis, ${ }^{44}$ A. Kyriakis, ${ }^{44}$ D. Loukas, ${ }^{44}$ G. Paspalaki, ${ }^{44}$ A. Agapitos, ${ }^{45}$ G. Karathanasis, ${ }^{45}$ P. Kontaxakis, ${ }^{45}$ A. Panagiotou, ${ }^{45}$ I. Papavergou, ${ }^{45}$ N. Saoulidou, ${ }^{45}$ K. Vellidis,${ }^{45}$ K. Kousouris ${ }^{46}$ I. Papakrivopoulos,${ }^{46}$ G. Tsipolitis, ${ }^{46}$ I. Evangelou, ${ }^{47}$ C. Foudas, ${ }^{47}$ P. Gianneios, ${ }^{47}$ P. Katsoulis, ${ }^{47}$ P. Kokkas, ${ }^{47}$ S. Mallios, ${ }^{47}$ N. Manthos,${ }^{47}$ I. Papadopoulos,${ }^{47}$ E. Paradas, ${ }^{47}$ J. Strologas, ${ }^{47}$ F. A. Triantis, ${ }^{47}$ D. Tsitsonis, ${ }^{47}$ M. Bartók, ${ }^{48, u}$ M. Csanad ${ }^{48}$ N. Filipovic, ${ }^{48}$ P. Major, ${ }^{48}$ M. I. Nagy, ${ }^{48}$ G. Pasztor, ${ }^{48}$ O. Surányi, ${ }^{48}$ G. I. Veres, ${ }^{48}$ G. Bencze, ${ }^{49}$ C. Hajdu ${ }^{49}$ D. Horvath ${ }^{49, v}$ Á. Hunyadi, ${ }^{49}$ F. Sikler, ${ }^{49}$ T. Á. Vámi, ${ }^{49}$ V. Veszpremi, ${ }^{49}$ G. Vesztergombi, ${ }^{49, a, w}$ N. Beni,${ }^{50}$ S. Czellar, ${ }^{50}$ J. Karancsi, ${ }^{50, u}$ A. Makovec, ${ }^{50}$ J. Molnar, ${ }^{50}$ Z. Szillasi, ${ }^{50}$ P. Raics ${ }^{51}$ Z. L. Trocsanyi, ${ }^{51}$ B. Ujvari, ${ }^{51}$ S. Choudhury, ${ }^{52}$ J. R. Komaragiri, ${ }^{52}$ P. C. Tiwari, ${ }^{52}$ S. Bahinipati, ${ }^{53, x}$ C. Kar,${ }^{53}$ P. Mal, ${ }^{53}$ K. Mandal, ${ }^{53}$ A. Nayak, ${ }^{53, y}$ S. Roy Chowdhury, ${ }^{53}$ D. K. Sahoo, ${ }^{53, x}$ S. K. Swain, ${ }^{53}$ S. Bansal, ${ }^{54}$ S. B. Beri, ${ }^{54}$ V. Bhatnagar, ${ }^{54}$ S. Chauhan, ${ }^{54}$ R. Chawla ${ }^{54}$ N. Dhingra, ${ }^{54}$ R. Gupta, ${ }^{54}$ A. Kaur, ${ }^{54}$ M. Kaur, ${ }^{54}$ S. Kaur, ${ }^{54}$ P. Kumari, ${ }^{54}$ M. Lohan, ${ }^{54}$ M. Meena, ${ }^{54}$ A. Mehta, ${ }^{54}$ K. Sandeep,${ }^{54}$ S. Sharma, ${ }^{54}$ J. B. Singh, ${ }^{54}$ A. K. Virdi, ${ }^{54}$ G. Walia, ${ }^{54}$ A. Bhardwaj, ${ }^{55}$ B. C. Choudhary, ${ }^{55}$ R. B. Garg, ${ }^{55}$ M. Gola,${ }^{55}$ S. Keshri,${ }^{55}$ Ashok Kumar, ${ }^{55}$ S. Malhotra, ${ }^{55}$ M. Naimuddin, ${ }^{55}$ P. Priyanka, ${ }^{55}$ K. Ranjan, ${ }^{55}$ Aashaq Shah, ${ }^{55}$ R. Sharma ${ }^{55}$ R. Bhardwaj,${ }^{56, z}$ M. Bharti, ${ }^{56, z}$ R. Bhattacharya ${ }^{56}$ S. Bhattacharya, ${ }^{56}$ U. Bhawandeep, ${ }^{56, z}$ D. Bhowmik, ${ }^{56}$ S. Dey, ${ }^{56}$ S. Dutt, ${ }^{56, z}$ S. Dutta, ${ }^{56}$ S. Ghosh,${ }^{56}$ M. Maity, ${ }^{56, a a}$ K. Mondal, ${ }^{56}$ S. Nandan, ${ }^{56}$ A. Purohit, ${ }^{56}$ P. K. Rout,${ }^{56}$ A. Roy, ${ }^{56}$ G. Saha, ${ }^{56}$ S. Sarkar, ${ }^{56}$ T. Sarkar,${ }^{56, a a}$ M. Sharan, ${ }^{56}$ B. Singh, ${ }^{56, z}$ S. Thakur, ${ }^{56, z}$ P. K. Behera, ${ }^{57}$ A. Muhammad,${ }^{57}$ R. Chudasama, ${ }^{58}$ D. Dutta, ${ }^{58}$ V. Jha, ${ }^{58}$ V. Kumar, ${ }^{58}$ D. K. Mishra, ${ }^{58}$ P. K. Netrakanti, ${ }^{58}$ L. M. Pant,${ }^{58}$ P. Shukla,${ }^{58}$ P. Suggisetti, ${ }^{58}$ T. Aziz, ${ }^{59}$ M. A. Bhat,${ }^{59}$ S. Dugad,${ }^{59}$ G. B. Mohanty, ${ }^{59}$ N. Sur, ${ }^{59}$ Ravindra Kumar Verma, ${ }^{59}$ S. Banerjee, ${ }^{60}$ S. Bhattacharya,${ }^{60}$ S. Chatterjee,${ }^{60}$ P. Das, ${ }^{60}$ M. Guchait, ${ }^{60}$ Sa. Jain, ${ }^{60}$ S. Karmakar, ${ }^{60}$ S. Kumar, ${ }^{60}$ G. Majumder,${ }^{60}$ K. Mazumdar,${ }^{60}$ N. Sahoo,${ }^{60}$ S. Chauhan,${ }^{61}$ S. Dube,${ }^{61}$ V. Hegde,${ }^{61}$ A. Kapoor,${ }^{61}$

K. Kothekar, ${ }^{61}$ S. Pandey, ${ }^{61}$ A. Rane,${ }^{61}$ A. Rastogi ${ }^{61}$ S. Sharma, ${ }^{61}$ S. Chenarani, ${ }^{62, b b}$ E. Eskandari Tadavani, ${ }^{62}$

S. M. Etesami, ${ }^{62, b b}$ M. Khakzad, ${ }^{62}$ M. Mohammadi Najafabadi, ${ }^{62}$ M. Naseri, ${ }^{62}$ F. Rezaei Hosseinabadi, ${ }^{62}$ B. Safarzadeh, ${ }^{62, c c}$ M. Zeinali, ${ }^{62}$ M. Felcini, ${ }^{63}$ M. Grunewald, ${ }^{63}$ M. Abbrescia,${ }^{64 a, 64 b}$ C. Calabria, ${ }^{64 a, 64 b}$ A. Colaleo, ${ }^{64 a}$ D. Creanza,${ }^{64,64 c}$ 
L. Cristella, ${ }^{64 a, 64 b}$ N. De Filippis, ${ }^{64 a, 64 c}$ M. De Palma, ${ }^{64 a, 64 b}$ A. Di Florio, ${ }^{64 a, 64 b}$ F. Errico, ${ }^{64 a, 64 b}$ L. Fiore, ${ }^{64 a}$ A. Gelmi, ${ }^{64 a, 64 b}$ G. Iaselli, ${ }^{64 a, 64 c}$ M. Ince, ${ }^{64 a, 64 b}$ S. Lezki, ${ }^{64 a, 64 b}$ G. Maggi, ${ }^{64 a, 64 c}$ M. Maggi, ${ }^{64 a}$ G. Miniello, ${ }^{64 a, 64 b}$ S. My, ${ }^{64 a, 64 b}$ S. Nuzzo, ${ }^{64 a, 64 b}$ A. Pompili, ${ }^{64 a, 64 b}$ G. Pugliese, ${ }^{64 a, 64 c}$ R. Radogna, ${ }^{64 a}$ A. Ranieri, ${ }^{64 a}$ G. Selvaggi, ${ }^{64 a, 64 b}$ A. Sharma, ${ }^{64 a}$ L. Silvestris, ${ }^{64 a}$

R. Venditti, ${ }^{64 a}$ P. Verwilligen, ${ }^{64 a}$ G. Abbiendi, ${ }^{65 a}$ C. Battilana, ${ }^{65 a, 65 b}$ D. Bonacorsi, ${ }^{65 a, 65 b}$ L. Borgonovi, ${ }^{65 a, 65 b}$

S. Braibant-Giacomelli, ${ }^{65 a, 65 b}$ R. Campanini, ${ }^{65 a, 65 b}$ P. Capiluppi, ${ }^{65 a, 65 b}$ A. Castro, ${ }^{65 a, 65 b}$ F. R. Cavallo, ${ }^{65 a}$ S. S. Chhibra, ${ }^{65 a, 65 b}$ G. Codispoti, ${ }^{65 a, 65 b}$ M. Cuffiani, ${ }^{65 a, 65 b}$ G. M. Dallavalle, ${ }^{65 a}$ F. Fabbri, ${ }^{65 a}$ A. Fanfani, ${ }^{65 a, 65 b}$ E. Fontanesi, ${ }^{65 a}$ P. Giacomelli, ${ }^{65 a}$ C. Grandi, ${ }^{65 a}$ L. Guiducci, ${ }^{65 a, 65 b}$ F. Iemmi, ${ }^{65 a, 65 b}$ S. Lo Meo, ${ }^{65 a, d d}$ S. Marcellini, ${ }^{65 a}$ G. Masetti, ${ }^{65 a}$ A. Montanari, ${ }^{65 a}$ F. L. Navarria, ${ }^{65 a, 65 b}$ A. Perrotta, ${ }^{65 a}$ F. Primavera, ${ }^{65 a, 65 b}$ A. M. Rossi, ${ }^{65 a, 65 b}$ T. Rovelli, ${ }^{65 a, 65 b}$ G. P. Siroli, ${ }^{65 a, 65 b}$ N. Tosi, ${ }^{65 a}$ S. Albergo, ${ }^{66 a, 66 b}$ A. Di Mattia, ${ }^{66 a}$ R. Potenza, ${ }^{66 a, 66 b}$ A. Tricomi, ${ }^{66 a, 66 b}$ C. Tuve, ${ }^{66 a, 66 b}$ G. Barbagli, ${ }^{67 a}$ K. Chatterjee, ${ }^{67 a, 67 b}$ V. Ciulli, ${ }^{67 a, 67 b}$ C. Civinini, ${ }^{67 a}$ R. D’Alessandro, ${ }^{67 a, 67 b}$ E. Focardi, ${ }^{67 a, 67 b}$ G. Latino, ${ }^{67 a}$ P. Lenzi, ${ }^{67 a, 67 b}$ M. Meschini, ${ }^{67 a}$ S. Paoletti, ${ }^{67 a}$ L. Russo, ${ }^{67 a, e e}$ G. Sguazzoni, ${ }^{67 a}$ D. Strom, ${ }^{67 a}$ L. Viliani, ${ }^{67 a}$ L. Benussi, ${ }^{68}$ S. Bianco, ${ }^{68}$ F. Fabbri, ${ }^{68}$ D. Piccolo, ${ }^{68}$ F. Ferro, ${ }^{69 a}$ R. Mulargia, ${ }^{69 a, 69 b}$ E. Robutti, ${ }^{69 a}$ S. Tosi, ${ }^{69 a, 69 b}$ A. Benaglia, ${ }^{70 a}$ A. Beschi, ${ }^{70 a, 70 b}$ F. Brivio, ${ }^{70 a, 70 b}$ V. Ciriolo, ${ }^{70 a, 70 b, q}$ S. Di Guida, ${ }^{70 a, 70 b, q}$ M. E. Dinardo, ${ }^{70 a, 70 b}$ S. Fiorendi, ${ }^{70 a, 70 b}$ S. Gennai, ${ }^{70 a}$ A. Ghezzi, ${ }^{70 a, 70 b}$ P. Govoni, ${ }^{70 a, 70 b}$ M. Malberti, ${ }^{70 a, 70 b}$

S. Malvezzi, ${ }^{70 a}$ D. Menasce, ${ }^{70 a}$ F. Monti, ${ }^{70 a}$ L. Moroni, ${ }^{70 a}$ M. Paganoni, ${ }^{70 a, 70 b}$ D. Pedrini, ${ }^{70 a}$ S. Ragazzi, $^{70 a, 70 b}$

T. Tabarelli de Fatis, ${ }^{70 a, 70 b}$ D. Zuolo, ${ }^{70 a, 70 b}$ S. Buontempo, ${ }^{71 a}$ N. Cavallo, ${ }^{71 a, 71 c}$ A. De Iorio, ${ }^{71 a, 71 b}$ A. Di Crescenzo, ${ }^{71 a, 71 b}$ F. Fabozzi, ${ }^{71 a, 71 c}$ F. Fienga, ${ }^{71 a}$ G. Galati, ${ }^{71 a}$ A. O. M. Iorio, ${ }^{71 a, 71 b}$ L. Lista, $^{71 a}$ S. Meola, ${ }^{71 a, 71 d, q}$ P. Paolucci, ${ }^{71 a, q}$

C. Sciacca, ${ }^{71 a, 71 b}$ E. Voevodina, ${ }^{71 a, 71 b}$ P. Azzi, ${ }^{72 a}$ N. Bacchetta, ${ }^{72 a}$ D. Bisello, ${ }^{72 a, 72 b}$ A. Boletti, ${ }^{72 a, 72 b}$ A. Bragagnolo, ${ }^{72 a}$

R. Carlin, ${ }^{72 a, 72 b}$ P. Checchia, ${ }^{72 a}$ M. Dall'Osso, ${ }^{72 a, 72 b}$ P. De Castro Manzano, ${ }^{72 a}$ T. Dorigo, ${ }^{72 a}$ U. Dosselli, $^{72 a}$

F. Gasparini, ${ }^{72 a, 72 b}$ U. Gasparini, ${ }^{72 a, 72 b}$ A. Gozzelino, ${ }^{72 a}$ S. Y. Hoh, ${ }^{72 a}$ S. Lacaprara, ${ }^{72 a}$ P. Lujan, ${ }^{72 a}$ M. Margoni, ${ }^{72 a, 72 b}$ A. T. Meneguzzo, ${ }^{72 a, 72 b}$ J. Pazzini, ${ }^{72 a, 72 b}$ M. Presilla, ${ }^{72 a, 72 b}$ P. Ronchese, ${ }^{72 a, 72 b}$ R. Rossin, ${ }^{72 a, 72 b}$ F. Simonetto, ${ }^{72 a, 72 b}$ A. Tiko, ${ }^{72 a}$ E. Torassa, ${ }^{72 a}$ M. Tosi, ${ }^{72 a, 72 b}$ M. Zanetti, ${ }^{72 a, 72 b}$ P. Zotto, ${ }^{72 a, 72 b}$ G. Zumerle, ${ }^{72 a, 72 b}$ A. Braghieri, ${ }^{73 a}$ A. Magnani, ${ }^{73 a}$ P. Montagna, ${ }^{73 a, 73 b}$ S. P. Ratti, ${ }^{73 a, 73 b}$ V. Re, ${ }^{73 a}$ M. Ressegotti, ${ }^{73 a, 73 b}$ C. Riccardi, ${ }^{73 a, 73 b}$ P. Salvini, ${ }^{73 a}$ I. Vai, $^{73 a, 73 b}$ P. Vitulo, ${ }^{73 a, 73 b}$ M. Biasini, ${ }^{74 a, 74 b}$ G. M. Bilei, ${ }^{74 a}$ C. Cecchi, ${ }^{74 a, 74 b}$ D. Ciangottini, ${ }^{74 a, 74 b}$ L. Fanò, ${ }^{74 a, 74 b}$ P. Lariccia, ${ }^{74 a, 74 b}$ R. Leonardi, ${ }^{74 a, 74 b}$ E. Manoni, ${ }^{74 a}$ G. Mantovani, ${ }^{74 a, 74 b}$ V. Mariani, ${ }^{74 a, 74 b}$ M. Menichelli, ${ }^{74 a}$ A. Rossi, ${ }^{74 a, 74 b}$ A. Santocchia, ${ }^{74 a, 74 b}$ D. Spiga, ${ }^{74 a}$ K. Androsov, ${ }^{75 a}$ P. Azzurri, ${ }^{75 a}$ G. Bagliesi, ${ }^{75 a}$ L. Bianchini, ${ }^{75 a}$ T. Boccali, ${ }^{75 a}$ L. Borrello, ${ }^{75 a}$ R. Castaldi, ${ }^{75 a}$ M. A. Ciocci, ${ }^{75 a, 75 b}$ R. Dell'Orso, ${ }^{75 a}$ G. Fedi, ${ }^{75 a}$ F. Fiori, ${ }^{75 a, 75 c}$ L. Giannini, ${ }^{75 a, 75 c}$ A. Giassi, ${ }^{75 a}$ M. T. Grippo, ${ }^{75 a}$ F. Ligabue, $^{75 a, 75 c}$ E. Manca, ${ }^{75 a, 75 c}$ G. Mandorli, ${ }^{75 a, 75 c}$ A. Messineo, ${ }^{75 a, 75 b}$ F. Palla, ${ }^{75 a}$ A. Rizzi, ${ }^{75 a, 75 b}$ G. Rolandi, ${ }^{75 a, f f}$ P. Spagnolo, ${ }^{75 a}$ R. Tenchini, ${ }^{75 a}$

G. Tonelli, ${ }^{75 a, 75 b}$ A. Venturi, ${ }^{75 a}$ P. G. Verdini, ${ }^{75 a}$ L. Barone, ${ }^{76 a, 76 b}$ F. Cavallari, ${ }^{76 a}$ M. Cipriani, ${ }^{76 a, 76 b}$ D. Del Re, ${ }^{76 a, 76 b}$ E. Di Marco, ${ }^{76 a, 76 b}$ M. Diemoz, ${ }^{76 a}$ S. Gelli, ${ }^{76 a, 76 b}$ E. Longo, ${ }^{76 a, 76 b}$ B. Marzocchi, ${ }^{76 a, 76 b}$ P. Meridiani, ${ }^{76 a}$ G. Organtini, ${ }^{76 a, 76 b}$ F. Pandolfi, ${ }^{76 a}$ R. Paramatti, ${ }^{76 a, 76 b}$ F. Preiato, ${ }^{76 a, 76 b}$ S. Rahatlou, ${ }^{76 a, 76 b}$ C. Rovelli, ${ }^{76 a}$ F. Santanastasio, ${ }^{76 a, 76 b}$ N. Amapane, ${ }^{77 a, 77 b}$ R. Arcidiacono, ${ }^{77 a, 77 c}$ S. Argiro, ${ }^{77 a, 77 b}$ M. Arneodo, ${ }^{77 a, 77 c}$ N. Bartosik, ${ }^{77 a}$ R. Bellan, ${ }^{77 a, 77 b}$ C. Biino, ${ }^{77 a}$ A. Cappati, ${ }^{77 a, 77 b}$ N. Cartiglia, ${ }^{77 a}$ F. Cenna, ${ }^{77 a, 77 b}$ S. Cometti, ${ }^{77 a}$ M. Costa, ${ }^{77,77 b}$ R. Covarelli, ${ }^{77 a, 77 b}$ N. Demaria, ${ }^{77 a}$ B. Kiani, ${ }^{77 a, 77 b}$ C. Mariotti, ${ }^{77 a}$ S. Maselli, ${ }^{77 a}$ E. Migliore, ${ }^{77 a, 77 b}$ V. Monaco, ${ }^{77 a, 77 b}$ E. Monteil, ${ }^{77 a, 77 b}$ M. Monteno, ${ }^{77 a}$ M. M. Obertino, ${ }^{77 a, 77 b}$ L. Pacher, ${ }^{77 a, 77 b}$ N. Pastrone, ${ }^{77 a}$ M. Pelliccioni, ${ }^{77 a}$ G. L. Pinna Angioni, ${ }^{77 a, 77 b}$ A. Romero, ${ }^{77 a, 77 b}$ M. Ruspa, ${ }^{77 a, 77 c}$

R. Sacchi, ${ }^{77 a, 77 b}$ R. Salvatico, ${ }^{77 a, 77 b}$ K. Shchelina, ${ }^{77 a, 77 b}$ V. Sola, ${ }^{77 a}$ A. Solano, ${ }^{77 a, 77 b}$ D. Soldi, ${ }^{77 a, 77 b}$ A. Staiano, ${ }^{77 a}$ S. Belforte, ${ }^{78 a}$ V. Candelise, ${ }^{78 a, 78 b}$ M. Casarsa, ${ }^{78 a}$ F. Cossutti, ${ }^{78 a}$ A. Da Rold, ${ }^{78 a, 78 b}$ G. Della Ricca, ${ }^{78 a, 78 b}$ F. Vazzoler, ${ }^{78 a, 78 b}$ A. Zanetti, ${ }^{78 a}$ D. H. Kim, ${ }^{79}$ G. N. Kim, ${ }^{79}$ M. S. Kim, ${ }^{79}$ J. Lee, ${ }^{79}$ S. Lee, ${ }^{79}$ S. W. Lee, ${ }^{79}$ C. S. Moon, ${ }^{79}$ Y. D. Oh, ${ }^{79}$ S. I. Pak, ${ }^{79}$ S. Sekmen, ${ }^{79}$ D. C. Son, ${ }^{79}$ Y. C. Yang, ${ }^{79}$ H. Kim, ${ }^{80}$ D. H. Moon, ${ }^{80}$ G. Oh, ${ }^{80}$ B. Francois, ${ }^{81}$ J. Goh, ${ }^{81, g g}$ T. J. Kim, ${ }^{81}$ S. Cho, ${ }^{82}$ S. Choi, ${ }^{82}$ Y. Go, ${ }^{82}$ D. Gyun, ${ }^{82}$ S. Ha, ${ }^{82}$ B. Hong, ${ }^{82}$ Y. Jo, ${ }^{82}$ K. Lee, ${ }^{82}$ K. S. Lee, ${ }^{82}$ S. Lee, ${ }^{82}$ J. Lim, $^{82}$ S. K. Park, ${ }^{82}$ Y. Roh, ${ }^{82}$ H. S. Kim, ${ }^{83}$ J. Almond, ${ }^{84}$ J. Kim, ${ }^{84}$ J. S. Kim, ${ }^{84}$ H. Lee, ${ }^{84}$ K. Lee, ${ }^{84}$ K. Nam, ${ }^{84}$ S. B. Oh, ${ }^{84}$ B. C. Radburn-Smith, ${ }^{84}$ S. h. Seo, ${ }^{84}$ U. K. Yang, ${ }^{84}$ H. D. Yoo, ${ }^{84}$ G. B. Yu, ${ }^{84}$ D. Jeon, ${ }^{85}$ H. Kim, ${ }^{85}$ J. H. Kim, ${ }^{85}$ J. S. H. Lee, ${ }^{85}$ I. C. Park, $^{85}$ Y. Choi, ${ }^{86}$ C. Hwang, ${ }^{86}$ J. Lee, ${ }^{86}$ I. Yu, ${ }^{86}$ V. Veckalns, ${ }^{87, \text { hh }}$ V. Dudenas, ${ }^{88}$ A. Juodagalvis, ${ }^{88}$ J. Vaitkus, ${ }^{88}$ Z. A. Ibrahim, ${ }^{89}$

M. A. B. Md Ali, ${ }^{89, i i}$ F. Mohamad Idris, ${ }^{89, j j}$ W. A. T. Wan Abdullah, ${ }^{89}$ M. N. Yusli, ${ }^{89}$ Z. Zolkapli, ${ }^{89}$ J. F. Benitez, ${ }^{90}$

A. Castaneda Hernandez, ${ }^{90}$ J. A. Murillo Quijada, ${ }^{90}$ H. Castilla-Valdez, ${ }^{91}$ E. De La Cruz-Burelo, ${ }^{91}$ M. C. Duran-Osuna, ${ }^{91}$ I. Heredia-De La Cruz, ${ }^{91, k k}$ R. Lopez-Fernandez, ${ }^{91}$ J. Mejia Guisao, ${ }^{91}$ R. I. Rabadan-Trejo, ${ }^{91}$ M. Ramirez-Garcia, ${ }^{91}$

G. Ramirez-Sanchez, ${ }^{91}$ R. Reyes-Almanza, ${ }^{91}$ A. Sanchez-Hernandez, ${ }^{91}$ S. Carrillo Moreno, ${ }^{92}$ C. Oropeza Barrera, ${ }^{92}$

F. Vazquez Valencia, ${ }^{92}$ J. Eysermans, ${ }^{93}$ I. Pedraza, ${ }^{93}$ H. A. Salazar Ibarguen, ${ }^{93}$ C. Uribe Estrada, ${ }^{93}$ A. Morelos Pineda, ${ }^{94}$ D. Krofcheck, ${ }^{95}$ S. Bheesette, ${ }^{96}$ P. H. Butler, ${ }^{96}$ A. Ahmad, ${ }^{97}$ M. Ahmad, ${ }^{97}$ M. I. Asghar, ${ }^{97}$ Q. Hassan, ${ }^{97}$ H. R. Hoorani, ${ }^{97}$ W. A. Khan, ${ }^{97}$ M. A. Shah, ${ }^{97}$ M. Shoaib, ${ }^{97}$ M. Waqas, ${ }^{97}$ H. Bialkowska, ${ }^{98}$ M. Bluj, ${ }^{98}$ B. Boimska, ${ }^{98}$ T. Frueboes, ${ }^{98}$ 
M. Górski, ${ }^{98}$ M. Kazana, ${ }^{98}$ M. Szleper,${ }^{98}$ P. Traczyk, ${ }^{98}$ P. Zalewski, ${ }^{98}$ K. Bunkowski, ${ }^{99}$ A. Byszuk, ${ }^{99,11}$ K. Doroba, ${ }^{99}$ A. Kalinowski, ${ }^{99}$ M. Konecki, ${ }^{99}$ J. Krolikowski, ${ }^{99}$ M. Misiura, ${ }^{99}$ M. Olszewski, ${ }^{99}$ A. Pyskir, ${ }^{99}$ M. Walczak, ${ }^{99}$ M. Araujo, ${ }^{100}$ P. Bargassa, ${ }^{100}$ C. Beirão Da Cruz E Silva, ${ }^{100}$ A. Di Francesco, ${ }^{100}$ P. Faccioli, ${ }^{100}$ B. Galinhas, ${ }^{100}$ M. Gallinaro,${ }^{100}$ J. Hollar, ${ }^{100}$ N. Leonardo, ${ }^{100}$ J. Seixas, ${ }^{100}$ G. Strong, ${ }^{100}$ O. Toldaiev,${ }^{100}$ J. Varela, ${ }^{100}$ S. Afanasiev ${ }^{101}$ P. Bunin, ${ }^{101}$ M. Gavrilenko, ${ }^{101}$ I. Golutvin, ${ }^{101}$ I. Gorbunov, ${ }^{101}$ A. Kamenev, ${ }^{101}$ V. Karjavine, ${ }^{101}$ A. Lanev, ${ }^{101}$ A. Malakhov, ${ }^{101}$ V. Matveev, ${ }^{101, m m, n n}$ P. Moisenz, ${ }^{101}$ V. Palichik, ${ }^{101}$ V. Perelygin, ${ }^{101}$ S. Shmatov, ${ }^{101}$ S. Shulha, ${ }^{101}$ N. Skatchkov, ${ }^{101}$ V. Smirnov, ${ }^{101}$ N. Voytishin, ${ }^{101}$ A. Zarubin, ${ }^{101}$ V. Golovtsov, ${ }^{102}$ Y. Ivanov, ${ }^{102}$ V. Kim, ${ }^{102,00}$ E. Kuznetsova, ${ }^{102, p p}$ P. Levchenko, ${ }^{102}$ V. Murzin, ${ }^{102}$ V. Oreshkin, ${ }^{102}$ I. Smirnov, ${ }^{102}$ D. Sosnov, ${ }^{102}$ V. Sulimov, ${ }^{102}$ L. Uvarov, ${ }^{102}$ S. Vavilov, ${ }^{102}$ A. Vorobyev, ${ }^{102}$ Yu. Andreev, ${ }^{103}$ A. Dermenev, ${ }^{103}$ S. Gninenko, ${ }^{103}$ N. Golubev, ${ }^{103}$ A. Karneyeu, ${ }^{103}$ M. Kirsanov, ${ }^{103}$ N. Krasnikov, ${ }^{103}$ A. Pashenkov, ${ }^{103}$ A. Shabanov, ${ }^{103}$ D. Tlisov, ${ }^{103}$ A. Toropin, ${ }^{103}$ V. Epshteyn, ${ }^{104}$ V. Gavrilov, ${ }^{104}$ N. Lychkovskaya, ${ }^{104}$ V. Popov, ${ }^{104}$ I. Pozdnyakov, ${ }^{104}$ G. Safronov, ${ }^{104}$ A. Spiridonov, ${ }^{104}$ A. Stepennov, ${ }^{104}$ V. Stolin, ${ }^{104}$ M. Toms, ${ }^{104}$ E. Vlasov, ${ }^{104}$ A. Zhokin, ${ }^{104}$ T. Aushev, ${ }^{105}$ R. Chistov, ${ }^{106, q q}$ M. Danilov, ${ }^{106, q q}$ S. Polikarpov, ${ }^{106, q q}$ E. Tarkovskii, ${ }^{106}$ V. Andreev, ${ }^{107}$ M. Azarkin, ${ }^{107}$ I. Dremin, ${ }^{107, n n}$ M. Kirakosyan, ${ }^{107}$ A. Terkulov, ${ }^{107}$ A. Baskakov, ${ }^{108}$ A. Belyaev,${ }^{108}$ E. Boos, ${ }^{108}$ V. Bunichev, ${ }^{108}$ M. Dubinin, ${ }^{108, \text { rr }}$ L. Dudko, ${ }^{108}$ A. Gribushin, ${ }^{108}$ V. Klyukhin, ${ }^{108}$ O. Kodolova, ${ }^{108}$ I. Lokhtin, ${ }^{108}$ S. Obraztsov, ${ }^{108}$ S. Petrushanko, ${ }^{108}$ V. Savrin, ${ }^{108}$ A. Barnyakov, ${ }^{109, \text { ss }}$ V. Blinov, ${ }^{109, \text { ss }}$ T. Dimova, ${ }^{109, s s}$ L. Kardapoltsev, ${ }^{109, s s}$ Y. Skovpen, ${ }^{109, s s}$ I. Azhgirey, ${ }^{110}$ I. Bayshev, ${ }^{10}$ S. Bitioukov, ${ }^{110}$ V. Kachanov, ${ }^{10}$ A. Kalinin, ${ }^{110}$ D. Konstantinov, ${ }^{110}$ P. Mandrik, ${ }^{110}$ V. Petrov, ${ }^{110}$ R. Ryutin, ${ }^{110}$ S. Slabospitskii, ${ }^{110}$ A. Sobol, ${ }^{110}$ S. Troshin, ${ }^{110}$ N. Tyurin, ${ }^{110}$ A. Uzunian, ${ }^{110}$ A. Volkov, ${ }^{110}$ A. Babaev, ${ }^{111}$ S. Baidali, ${ }^{111}$ V. Okhotnikov, ${ }^{111}$ P. Adzic, ${ }^{112, t \mathrm{t}}$ P. Cirkovic, ${ }^{112}$ D. Devetak, ${ }^{112}$ M. Dordevic, ${ }^{112}$ P. Milenovic, ${ }^{11, \text { uu }}$ J. Milosevic, ${ }^{112}$ J. Alcaraz Maestre, ${ }^{113}$ A. Álvarez Fernández, ${ }^{113}$ I. Bachiller, ${ }^{113}$ M. Barrio Luna, ${ }^{113}$

J. A. Brochero Cifuentes, ${ }^{113}$ M. Cerrada, ${ }^{113}$ N. Colino, ${ }^{113}$ B. De La Cruz, ${ }^{113}$ A. Delgado Peris, ${ }^{113}$ C. Fernandez Bedoya, ${ }^{113}$

J. P. Fernández Ramos, ${ }^{113}$ J. Flix, ${ }^{113}$ M. C. Fouz, ${ }^{113}$ O. Gonzalez Lopez, ${ }^{113}$ S. Goy Lopez, ${ }^{113}$ J. M. Hernandez, ${ }^{113}$

M. I. Josa, ${ }^{113}$ D. Moran, ${ }^{113}$ A. Pérez-Calero Yzquierdo, ${ }^{113}$ J. Puerta Pelayo, ${ }^{113}$ I. Redondo, ${ }^{113}$ L. Romero, ${ }^{113}$

S. Sánchez Navas, ${ }^{113}$ M. S. Soares, ${ }^{113}$ A. Triossi, ${ }^{113}$ C. Albajar, ${ }^{114}$ J. F. de Trocóniz, ${ }^{114}$ J. Cuevas, ${ }^{115}$ C. Erice, ${ }^{115}$ J. Fernandez Menendez, ${ }^{115}$ S. Folgueras, ${ }^{115}$ I. Gonzalez Caballero, ${ }^{115}$ J. R. González Fernández, ${ }^{115}$ E. Palencia Cortezon, ${ }^{115}$ V. Rodríguez Bouza, ${ }^{115}$ S. Sanchez Cruz, ${ }^{115}$ J. M. Vizan Garcia, ${ }^{115}$ I. J. Cabrillo, ${ }^{116}$ A. Calderon, ${ }^{116}$ B. Chazin Quero, ${ }^{116}$ J. Duarte Campderros, ${ }^{116}$ M. Fernandez, ${ }^{116}$ P. J. Fernández Manteca, ${ }^{116}$ A. García Alonso, ${ }^{116}$ J. Garcia-Ferrero, ${ }^{116}$

G. Gomez, ${ }^{116}$ A. Lopez Virto, ${ }^{16}$ J. Marco, ${ }^{116}$ C. Martinez Rivero, ${ }^{116}$ P. Martinez Ruiz del Arbol, ${ }^{116}$ F. Matorras, ${ }^{116}$

J. Piedra Gomez, ${ }^{116}$ C. Prieels, ${ }^{116}$ T. Rodrigo, ${ }^{116}$ A. Ruiz-Jimeno, ${ }^{116}$ L. Scodellaro, ${ }^{116}$ N. Trevisani, ${ }^{116}$ I. Vila, ${ }^{116}$ R. Vilar Cortabitarte, ${ }^{116}$ N. Wickramage, ${ }^{117}$ D. Abbaneo, ${ }^{118}$ B. Akgun, ${ }^{118}$ E. Auffray, ${ }^{118}$ G. Auzinger, ${ }^{118}$ P. Baillon, ${ }^{118}$ A. H. Ball, ${ }^{118}$ D. Barney, ${ }^{118}$ J. Bendavid, ${ }^{118}$ M. Bianco, ${ }^{118}$ A. Bocci, ${ }^{118}$ C. Botta, ${ }^{118}$ E. Brondolin, ${ }^{118}$ T. Camporesi, ${ }^{118}$ M. Cepeda, ${ }^{118}$ G. Cerminara, ${ }^{118}$ E. Chapon, ${ }^{118}$ Y. Chen, ${ }^{118}$ G. Cucciati,${ }^{118}$ D. d'Enterria, ${ }^{118}$ A. Dabrowski, ${ }^{118}$ N. Daci, ${ }^{118}$ V. Daponte, ${ }^{118}$ A. David, ${ }^{118}$ A. De Roeck, ${ }^{118}$ N. Deelen, ${ }^{118}$ M. Dobson, ${ }^{118}$ M. Dünser, ${ }^{118}$ N. Dupont, ${ }^{118}$ A. Elliott-Peisert, ${ }^{118}$

F. Fallavollita, ${ }^{118, v v}$ D. Fasanella, ${ }^{118}$ G. Franzoni, ${ }^{118}$ J. Fulcher, ${ }^{118}$ W. Funk, ${ }^{118}$ D. Gigi, ${ }^{118}$ A. Gilbert, ${ }^{118}$ K. Gill,,${ }^{118}$

F. Glege, ${ }^{118}$ M. Gruchala, ${ }^{118}$ M. Guilbaud, ${ }^{118}$ D. Gulhan, ${ }^{118}$ J. Hegeman, ${ }^{118}$ C. Heidegger, ${ }^{118}$ V. Innocente, ${ }^{118}$ G. M. Innocenti, ${ }^{118}$ A. Jafari, ${ }^{118}$ P. Janot, ${ }^{118}$ O. Karacheban, ${ }^{118, t}$ J. Kieseler, ${ }^{118}$ A. Kornmayer, ${ }^{118}$ M. Krammer, ${ }^{118, b}$ C. Lange ${ }^{118}$ P. Lecoq, ${ }^{118}$ C. Lourenço, ${ }^{118}$ L. Malgeri, ${ }^{118}$ M. Mannelli, ${ }^{118}$ A. Massironi, ${ }^{118}$ F. Meijers,${ }^{118}$ J. A. Merlin, ${ }^{118}$ S. Mersi, ${ }^{118}$ E. Meschi, ${ }^{118}$ F. Moortgat, ${ }^{118}$ M. Mulders, ${ }^{118}$ J. Ngadiuba, ${ }^{118}$ S. Nourbakhsh, ${ }^{118}$ S. Orfanelli, ${ }^{118}$ L. Orsini, ${ }^{118}$ F. Pantaleo, ${ }^{118, q}$ L. Pape, ${ }^{118}$ E. Perez,${ }^{118}$ M. Peruzzi, ${ }^{118}$ A. Petrilli, ${ }^{118}$ G. Petrucciani ${ }^{118}$ A. Pfeiffer, ${ }^{118}$ M. Pierini, ${ }^{118}$ F. M. Pitters, ${ }^{118}$ D. Rabady, ${ }^{118}$ A. Racz, ${ }^{118}$ M. Rovere, ${ }^{118}$ H. Sakulin, ${ }^{118}$ C. Schäfer,${ }^{118}$ C. Schwick, ${ }^{118}$ M. Selvaggi, ${ }^{118}$ A. Sharma, ${ }^{118}$ P. Silva, ${ }^{118}$ P. Sphicas, ${ }^{118, w w}$ A. Stakia, ${ }^{118}$ J. Steggemann, ${ }^{118}$ D. Treille, ${ }^{118}$ A. Tsirou ${ }^{118}$ A. Vartak, ${ }^{118}$ M. Verzetti, ${ }^{118}$ W. D. Zeuner, ${ }^{118}$ L. Caminada, ${ }^{11, x x}$ K. Deiters, ${ }^{119}$ W. Erdmann, ${ }^{119}$ R. Horisberger, ${ }^{119}$ Q. Ingram, ${ }^{119}$ H. C. Kaestli, ${ }^{119}$ D. Kotlinski, ${ }^{119}$ U. Langenegger, ${ }^{119}$ T. Rohe, ${ }^{119}$ S. A. Wiederkehr, ${ }^{119}$ M. Backhaus, ${ }^{120}$ L. Bäni, ${ }^{120}$ P. Berger ${ }^{120}$ N. Chernyavskaya, ${ }^{120}$ G. Dissertori, ${ }^{120}$ M. Dittmar, ${ }^{120}$ M. Donegà ${ }^{120}$ C. Dorfer, ${ }^{120}$ T. A. Gómez Espinosa, ${ }^{120}$ C. Grab,${ }^{120}$ D. Hits, ${ }^{120}$ T. Klijnsma, ${ }^{120}$ W. Lustermann, ${ }^{120}$ R. A. Manzoni, ${ }^{120}$ M. Marionneau, ${ }^{120}$ M. T. Meinhard, ${ }^{120}$ F. Micheli, ${ }^{120}$ P. Musella, ${ }^{120}$ F. Nessi-Tedaldi, ${ }^{120}$ F. Pauss, ${ }^{120}$ G. Perrin, ${ }^{120}$ L. Perrozzi, ${ }^{120}$ S. Pigazzini, ${ }^{120}$ M. Reichmann, ${ }^{120}$ C. Reissel, ${ }^{120}$ D. Ruini, ${ }^{120}$ D. A. Sanz Becerra, ${ }^{120}$ M. Schönenberger ${ }^{120}$ L. Shchutska, ${ }^{120}$ V. R. Tavolaro, ${ }^{120}$

K. Theofilatos, ${ }^{120}$ M. L. Vesterbacka Olsson, ${ }^{120}$ R. Wallny, ${ }^{120}$ D. H. Zhu, ${ }^{120}$ T. K. Aarrestad,${ }^{121}$ C. Amsler, ${ }^{121, y y}$

D. Brzhechko, ${ }^{121}$ M. F. Canelli, ${ }^{121}$ A. De Cosa,${ }^{121}$ R. Del Burgo, ${ }^{121}$ S. Donato, ${ }^{121}$ C. Galloni, ${ }^{121}$ T. Hreus, ${ }^{121}$

B. Kilminster, ${ }^{121}$ S. Leontsinis, ${ }^{121}$ I. Neutelings,${ }^{121}$ G. Rauco, ${ }^{121}$ P. Robmann, ${ }^{121}$ D. Salerno, ${ }^{121}$ K. Schweiger, ${ }^{121}$ C. Seitz, ${ }^{121}$ 
Y. Takahashi, ${ }^{121}$ S. Wertz, ${ }^{121}$ A. Zucchetta, ${ }^{121}$ T. H. Doan, ${ }^{122}$ R. Khurana, ${ }^{122}$ C. M. Kuo, ${ }^{122}$ W. Lin, ${ }^{122}$ S. S. Yu, ${ }^{122}$ P. Chang, ${ }^{123}$ Y. Chao, ${ }^{123}$ K. F. Chen, ${ }^{123}$ P. H. Chen, ${ }^{123}$ W.-S. Hou, ${ }^{123}$ Y. F. Liu, ${ }^{123}$ R.-S. Lu, ${ }^{123}$ E. Paganis, ${ }^{123}$ A. Psallidas, ${ }^{123}$ A. Steen, ${ }^{123}$ B. Asavapibhop, ${ }^{124}$ N. Srimanobhas, ${ }^{124}$ N. Suwonjandee, ${ }^{124}$ A. Bat, ${ }^{125}$ F. Boran, ${ }^{125}$ S. Cerci, ${ }^{125, z z}$ S. Damarseckin, ${ }^{125}$ Z. S. Demiroglu, ${ }^{125}$ F. Dolek, ${ }^{125}$ C. Dozen, ${ }^{125}$ I. Dumanoglu, ${ }^{125}$ G. Gokbulut, ${ }^{125}$ Y. Guler, ${ }^{125}$ E. Gurpinar, ${ }^{125}$ I. Hos, ${ }^{125}$,aaa C. Isik, ${ }^{125}$ E. E. Kangal, ${ }^{125, \text { bbb }}$ O. Kara, ${ }^{125}$ A. Kayis Topaksu, ${ }^{125}$ U. Kiminsu, ${ }^{125}$ M. Oglakci, ${ }^{125}$ G. Onengut, ${ }^{125}$ K. Ozdemir, ${ }^{125, \text { ccc }}$ S. Ozturk, ${ }^{125, \text { ddd }}$ D. Sunar Cerci, ${ }^{125, z z}$ B. Tali, ${ }^{125, z z}$ U. G. Tok, ${ }^{125}$ S. Turkcapar, ${ }^{125}$ I. S. Zorbakir, ${ }^{125}$ C. Zorbilmez, ${ }^{125}$ B. Isildak, ${ }^{126, e e e}$ G. Karapinar, ${ }^{126, f f f}$ M. Yalvac, ${ }^{126}$ M. Zeyrek, ${ }^{126}$ I. O. Atakisi, ${ }^{127}$ E. Gülmez, ${ }^{127}$ M. Kaya, ${ }^{127, g g g}$ O. Kaya, ${ }^{127, \text { hhh }}$ S. Ozkorucuklu, ${ }^{127, \text { iii }}$ S. Tekten, ${ }^{127}$ E. A. Yetkin, ${ }^{127, j j j}$ M. N. Agaras, ${ }^{128}$ A. Cakir, ${ }^{128}$ K. Cankocak, ${ }^{128}$ Y. Komurcu, ${ }^{128}$ S. Sen, ${ }^{128, k k k}$ B. Grynyov, ${ }^{129}$ L. Levchuk, ${ }^{130}$ F. Ball, ${ }^{131}$ J. J. Brooke, ${ }^{131}$ D. Burns, ${ }^{131}$ E. Clement, ${ }^{131}$ D. Cussans, ${ }^{131}$ O. Davignon, ${ }^{131}$ H. Flacher, ${ }^{131}$ J. Goldstein, ${ }^{131}$ G. P. Heath, ${ }^{131}$ H. F. Heath, ${ }^{131}$ L. Kreczko, ${ }^{131}$ D. M. Newbold, ${ }^{131,111}$ S. Paramesvaran, ${ }^{131}$ B. Penning, ${ }^{131}$ T. Sakuma, ${ }^{131}$ D. Smith, ${ }^{131}$ V. J. Smith, ${ }^{131}$ J. Taylor, ${ }^{131}$ A. Titterton, ${ }^{131}$ K. W. Bell, ${ }^{132}$ A. Belyaev, ${ }^{132, m m m}$ C. Brew,${ }^{132}$ R. M. Brown, ${ }^{132}$ D. Cieri, ${ }^{132}$ D. J. A. Cockerill, ${ }^{132}$ J. A. Coughlan, ${ }^{132}$ K. Harder, ${ }^{132}$ S. Harper, ${ }^{132}$ J. Linacre, ${ }^{132}$ K. Manolopoulos, ${ }^{132}$ E. Olaiya, ${ }^{132}$ D. Petyt, ${ }^{132}$ T. Reis, ${ }^{132}$ T. Schuh, ${ }^{132}$ C. H. Shepherd-Themistocleous, ${ }^{132}$ A. Thea, ${ }^{132}$ I. R. Tomalin, ${ }^{132}$ T. Williams, ${ }^{132}$ W. J. Womersley, ${ }^{132}$ R. Bainbridge ${ }^{133}$ P. Bloch, ${ }^{133}$ J. Borg, ${ }^{133}$ S. Breeze, ${ }^{133}$ O. Buchmuller ${ }^{133}$ A. Bundock, ${ }^{133}$ D. Colling, ${ }^{133}$ P. Dauncey,${ }^{133}$ G. Davies, ${ }^{133}$ M. Della Negra, ${ }^{133}$ R. Di Maria, ${ }^{133}$ P. Everaerts, ${ }^{133}$ G. Hall,${ }^{133}$ G. Iles, ${ }^{133}$ T. James, ${ }^{133}$ M. Komm, ${ }^{133}$ C. Laner, ${ }^{133}$ L. Lyons, ${ }^{133}$ A.-M. Magnan, ${ }^{133}$ S. Malik, ${ }^{133}$ A. Martelli, ${ }^{133}$ J. Nash, ${ }^{133, n n n}$ A. Nikitenko, ${ }^{133, \mathrm{~h}}$ V. Palladino, ${ }^{133}$ M. Pesaresi, ${ }^{133}$ D. M. Raymond, ${ }^{133}$ A. Richards, ${ }^{133}$ A. Rose, ${ }^{133}$ E. Scott, ${ }^{133}$ C. Seez, ${ }^{133}$ A. Shtipliyski, ${ }^{133}$ G. Singh, ${ }^{133}$ M. Stoye ${ }^{133}$ T. Strebler, ${ }^{133}$ S. Summers, ${ }^{133}$ A. Tapper, ${ }^{133}$ K. Uchida, ${ }^{133}$ T. Virdee, ${ }^{133, q}$ N. Wardle, ${ }^{133}$ D. Winterbottom, ${ }^{133}$ J. Wright, ${ }^{133}$ S. C. Zenz, ${ }^{133}$ J. E. Cole, ${ }^{134}$ P. R. Hobson, ${ }^{134}$ A. Khan, ${ }^{134}$ P. Kyberd, ${ }^{134}$ C. K. Mackay, ${ }^{134}$ A. Morton, ${ }^{134}$ I. D. Reid, ${ }^{134}$ L. Teodorescu, ${ }^{134}$ S. Zahid, ${ }^{134}$ K. Call, ${ }^{135}$ J. Dittmann, ${ }^{135}$ K. Hatakeyama, ${ }^{135}$ H. Liu, ${ }^{135}$ C. Madrid, ${ }^{135}$ B. McMaster, ${ }^{135}$ N. Pastika, ${ }^{135}$ C. Smith, ${ }^{135}$ R. Bartek, ${ }^{136}$ A. Dominguez, ${ }^{136}$ A. Buccilli, ${ }^{137}$ S. I. Cooper, ${ }^{137}$ C. Henderson, ${ }^{137}$ P. Rumerio, ${ }^{137}$ C. West, ${ }^{137}$ D. Arcaro, ${ }^{138}$ T. Bose,${ }^{138}$ Z. Demiragli, ${ }^{138}$ D. Gastler, ${ }^{138}$ S. Girgis, ${ }^{138}$ D. Pinna, ${ }^{138}$ C. Richardson, ${ }^{138}$ J. Rohlf, ${ }^{138}$ D. Sperka, ${ }^{138}$ I. Suarez, ${ }^{138}$ L. Sulak, ${ }^{138}$ D. Zou, ${ }^{138}$ G. Benelli, ${ }^{139}$ B. Burkle, ${ }^{139}$ X. Coubez, ${ }^{139}$ D. Cutts, ${ }^{139}$ M. Hadley, ${ }^{139}$ J. Hakala, ${ }^{139}$ U. Heintz, ${ }^{139}$ J. M. Hogan, ${ }^{139,000}$ K. H. M. Kwok, ${ }^{139}$ E. Laird, ${ }^{139}$ G. Landsberg, ${ }^{139}$ J. Lee, ${ }^{139}$ Z. Mao, ${ }^{139}$ M. Narain, ${ }^{139}$ S. Sagir, ${ }^{139, p p p}$ R. Syarif, ${ }^{139}$ E. Usai, ${ }^{139}$ D. Yu, ${ }^{139}$ R. Band, ${ }^{140}$ C. Brainerd, ${ }^{140}$ R. Breedon, ${ }^{140}$ D. Burns, ${ }^{140}$ M. Calderon De La Barca Sanchez, ${ }^{140}$ M. Chertok, ${ }^{140}$ J. Conway, ${ }^{140}$ R. Conway, ${ }^{140}$ P. T. Cox, ${ }^{140}$ R. Erbacher, ${ }^{140}$ C. Flores, ${ }^{140}$ G. Funk, ${ }^{140}$ W. Ko, ${ }^{140}$ O. Kukral, ${ }^{140}$ R. Lander, ${ }^{140}$ M. Mulhearn, ${ }^{140}$ D. Pellett, ${ }^{140}$ J. Pilot, ${ }^{140}$ S. Shalhout, ${ }^{140}$ M. Shi, ${ }^{140}$ D. Stolp, ${ }^{140}$ D. Taylor, ${ }^{140}$ K. Tos, ${ }^{140}$ M. Tripathi, ${ }^{140}$ Z. Wang, ${ }^{140}$ F. Zhang, ${ }^{140}$ M. Bachtis, ${ }^{141}$ C. Bravo, ${ }^{141}$ R. Cousins, ${ }^{141}$ A. Dasgupta, ${ }^{141}$ S. Erhan, ${ }^{141}$ A. Florent, ${ }^{141}$ J. Hauser ${ }^{141}$ M. Ignatenko, ${ }^{141}$ N. Mccoll, ${ }^{141}$ S. Regnard, ${ }^{141}$ D. Saltzberg, ${ }^{141}$ C. Schnaible, ${ }^{141}$ V. Valuev, ${ }^{141}$ E. Bouvier, ${ }^{142}$ K. Burt, ${ }^{142}$ R. Clare, ${ }^{142}$ J. W. Gary, ${ }^{142}$ S. M. A. Ghiasi Shirazi, ${ }^{142}$ G. Hanson, ${ }^{142}$ G. Karapostoli, ${ }^{142}$ E. Kennedy, ${ }^{142}$ F. Lacroix, ${ }^{142}$ O. R. Long, ${ }^{142}$

M. Olmedo Negrete, ${ }^{142}$ M. I. Paneva, ${ }^{142}$ W. Si,${ }^{142}$ L. Wang, ${ }^{142}$ H. Wei, ${ }^{142}$ S. Wimpenny, ${ }^{142}$ B. R. Yates, ${ }^{142}$ J. G. Branson, ${ }^{143}$ P. Chang, ${ }^{143}$ S. Cittolin, ${ }^{143}$ M. Derdzinski, ${ }^{143}$ R. Gerosa,${ }^{143}$ D. Gilbert, ${ }^{143}$ B. Hashemi, ${ }^{143}$ A. Holzner, ${ }^{143}$ D. Klein, ${ }^{143}$ G. Kole, ${ }^{143}$ V. Krutelyov, ${ }^{143}$ J. Letts, ${ }^{143}$ M. Masciovecchio, ${ }^{143}$ S. May, ${ }^{143}$ D. Olivito, ${ }^{143}$ S. Padhi, ${ }^{143}$ M. Pieri, ${ }^{143}$ V. Sharma, ${ }^{143}$ M. Tadel,${ }^{143}$ J. Wood, ${ }^{143}$ F. Würthwein, ${ }^{143}$ A. Yagil, ${ }^{143}$ G. Zevi Della Porta, ${ }^{143}$ N. Amin, ${ }^{144}$ R. Bhandari, ${ }^{144}$ C. Campagnari, ${ }^{144}$ M. Citron, ${ }^{144}$ V. Dutta, ${ }^{144}$ M. Franco Sevilla, ${ }^{144}$ L. Gouskos, ${ }^{144}$ R. Heller, ${ }^{144}$ J. Incandela, ${ }^{144}$ H. Mei, ${ }^{144}$ A. Ovcharova, ${ }^{144}$ H. Qu, ${ }^{144}$ J. Richman, ${ }^{144}$ D. Stuart, ${ }^{144}$ S. Wang, ${ }^{144}$ J. Yoo, ${ }^{144}$ D. Anderson, ${ }^{145}$ A. Bornheim, ${ }^{145}$ J. M. Lawhorn, ${ }^{145}$ N. Lu, ${ }^{145}$ H. B. Newman, ${ }^{145}$ T. Q. Nguyen, ${ }^{145}$ J. Pata, ${ }^{145}$ M. Spiropulu, ${ }^{145}$ J. R. Vlimant, ${ }^{145}$ R. Wilkinson, ${ }^{145}$ S. Xie, ${ }^{145}$ Z. Zhang, ${ }^{145}$ R. Y. Zhu, ${ }^{145}$ M. B. Andrews, ${ }^{146}$ T. Ferguson, ${ }^{146}$ T. Mudholkar, ${ }^{146}$ M. Paulini, ${ }^{146}$ M. Sun, ${ }^{146}$ I. Vorobiev, ${ }^{146}$ M. Weinberg, ${ }^{146}$ J. P. Cumalat, ${ }^{147}$ W. T. Ford, ${ }^{147}$ F. Jensen, ${ }^{147}$ A. Johnson, ${ }^{147}$ E. MacDonald, ${ }^{147}$ T. Mulholland ${ }^{147}$ R. Patel, ${ }^{147}$ A. Perloff, ${ }^{147}$ K. Stenson, ${ }^{147}$ K. A. Ulmer, ${ }^{147}$ S. R. Wagner, ${ }^{147}$ J. Alexander, ${ }^{148}$ J. Chaves, ${ }^{148}$ Y. Cheng, ${ }^{148}$ J. Chu, ${ }^{148}$ A. Datta, ${ }^{148}$ K. Mcdermott, ${ }^{148}$ N. Mirman, ${ }^{148}$ J. R. Patterson, ${ }^{148}$ D. Quach, ${ }^{148}$ A. Rinkevicius, ${ }^{148}$ A. Ryd ${ }^{148}$ L. Skinnari, ${ }^{148}$ L. Soffi, ${ }^{148}$ S. M. Tan, ${ }^{148}$ Z. Tao, ${ }^{148}$ J. Thom, ${ }^{148}$ J. Tucker, ${ }^{148}$ P. Wittich, ${ }^{148}$ M. Zientek, ${ }^{148}$ S. Abdullin, ${ }^{149}$ M. Albrow, ${ }^{149}$ M. Alyari, ${ }^{149}$ G. Apollinari, ${ }^{149}$ A. Apresyan, ${ }^{149}$ A. Apyan, ${ }^{149}$ S. Banerjee, ${ }^{149}$ L. A. T. Bauerdick, ${ }^{149}$ A. Beretvas, ${ }^{149}$ J. Berryhill, ${ }^{149}$ P. C. Bhat, ${ }^{149}$ K. Burkett, ${ }^{149}$ J. N. Butler, ${ }^{149}$ A. Canepa, ${ }^{149}$ G. B. Cerati, ${ }^{149}$ H. W. K. Cheung, ${ }^{149}$ F. Chlebana, ${ }^{149}$ M. Cremonesi, ${ }^{149}$ J. Duarte, ${ }^{149}$ V. D. Elvira, ${ }^{149}$ J. Freeman, ${ }^{149}$ Z. Gecse, ${ }^{149}$ E. Gottschalk, ${ }^{149}$ L. Gray, ${ }^{149}$ D. Green, ${ }^{149}$ S. Grünendahl, ${ }^{149}$ O. Gutsche, ${ }^{149}$ J. Hanlon, ${ }^{149}$ R. M. Harris, ${ }^{149}$ S. Hasegawa, ${ }^{149}$ J. Hirschauer, ${ }^{149}$ Z. Hu, ${ }^{149}$ B. Jayatilaka, ${ }^{149}$ S. Jindariani, ${ }^{149}$ M. Johnson, ${ }^{149}$ U. Joshi, ${ }^{149}$ B. Klima, ${ }^{149}$ 
M. J. Kortelainen, ${ }^{149}$ B. Kreis, ${ }^{149}$ S. Lammel, ${ }^{149}$ D. Lincoln, ${ }^{149}$ R. Lipton, ${ }^{149}$ M. Liu, ${ }^{149}$ T. Liu, ${ }^{149}$ J. Lykken, ${ }^{149}$ K. Maeshima, ${ }^{149}$ J. M. Marraffino, ${ }^{149}$ D. Mason, ${ }^{149}$ P. McBride, ${ }^{149}$ P. Merkel, ${ }^{149}$ S. Mrenna, ${ }^{149}$ S. Nahn, ${ }^{149}$ V. O’Dell, ${ }^{149}$ K. Pedro, ${ }^{149}$ C. Pena, ${ }^{149}$ O. Prokofyev, ${ }^{149}$ G. Rakness, ${ }^{149}$ F. Ravera, ${ }^{149}$ A. Reinsvold, ${ }^{149}$ L. Ristori, ${ }^{149}$ A. Savoy-Navarro, ${ }^{149, \mathrm{qqq}}$ B. Schneider, ${ }^{149}$ E. Sexton-Kennedy, ${ }^{149}$ A. Soha, ${ }^{149}$ W. J. Spalding, ${ }^{149}$ L. Spiegel, ${ }^{149}$ S. Stoynev, ${ }^{149}$ J. Strait, ${ }^{149}$ N. Strobbe, ${ }^{149}$ L. Taylor, ${ }^{149}$ S. Tkaczyk, ${ }^{149}$ N. V. Tran, ${ }^{149}$ L. Uplegger, ${ }^{149}$ E. W. Vaandering, ${ }^{149}$ C. Vernieri, ${ }^{149}$ M. Verzocchi, ${ }^{149}$ R. Vidal, ${ }^{149}$ M. Wang, ${ }^{149}$ H. A. Weber, ${ }^{149}$ D. Acosta, ${ }^{150}$ P. Avery, ${ }^{150}$ P. Bortignon, ${ }^{150}$ D. Bourilkov, ${ }^{150}$ A. Brinkerhoff, ${ }^{150}$ L. Cadamuro, ${ }^{150}$ A. Carnes, ${ }^{150}$ D. Curry, ${ }^{150}$ R. D. Field, ${ }^{150}$ S. V. Gleyzer, ${ }^{150}$ B. M. Joshi, ${ }^{150}$ J. Konigsberg, ${ }^{150}$ A. Korytov, ${ }^{150}$ K. H. Lo, ${ }^{150}$ P. Ma, ${ }^{150}$ K. Matchev, ${ }^{150}$ N. Menendez, ${ }^{150}$ G. Mitselmakher, ${ }^{150}$ D. Rosenzweig, ${ }^{150}$ K. Shi, ${ }^{150}$ J. Wang, ${ }^{150}$ S. Wang, ${ }^{150}$ X. Zuo, ${ }^{150}$ Y. R. Joshi, ${ }^{151}$ S. Linn, ${ }^{151}$ A. Ackert, ${ }^{152}$ T. Adams, ${ }^{152}$ A. Askew, ${ }^{152}$ S. Hagopian, ${ }^{152}$ V. Hagopian, ${ }^{152}$ K. F. Johnson, ${ }^{152}$ T. Kolberg, ${ }^{152}$ G. Martinez, ${ }^{152}$ T. Perry, ${ }^{152}$ H. Prosper, ${ }^{152}$ A. Saha, ${ }^{152}$ C. Schiber, ${ }^{152}$ R. Yohay, ${ }^{152}$ M. M. Baarmand, ${ }^{153}$ V. Bhopatkar, ${ }^{153}$ S. Colafranceschi, ${ }^{153}$ M. Hohlmann, ${ }^{153}$ D. Noonan, ${ }^{153}$ M. Rahmani, ${ }^{153}$ T. Roy, ${ }^{153}$ M. Saunders, ${ }^{153}$ F. Yumiceva, ${ }^{153}$ M. R. Adams, ${ }^{154}$ L. Apanasevich,${ }^{154}$ D. Berry, ${ }^{154}$ R. R. Betts, ${ }^{154}$ R. Cavanaugh, ${ }^{154}$ X. Chen, ${ }^{154}$ S. Dittmer, ${ }^{154}$ O. Evdokimov, ${ }^{154}$ C. E. Gerber, ${ }^{154}$ D. A. Hangal, ${ }^{154}$ D. J. Hofman, ${ }^{154}$ K. Jung, ${ }^{154}$ J. Kamin, ${ }^{154}$ C. Mills, ${ }^{154}$ M. B. Tonjes, ${ }^{154}$ N. Varelas, ${ }^{154}$ H. Wang, ${ }^{154}$ X. Wang, ${ }^{154}$ Z. Wu, ${ }^{154}$ J. Zhang, ${ }^{154}$ M. Alhusseini, ${ }^{155}$ B. Bilki, ${ }^{155, \text { rrr }}$ W. Clarida, ${ }^{155}$ K. Dilsiz, ${ }^{155, s s s}$ S. Durgut, ${ }^{155}$ R. P. Gandrajula, ${ }^{155}$ M. Haytmyradov, ${ }^{155}$ V. Khristenko, ${ }^{155}$ J.-P. Merlo, ${ }^{155}$ A. Mestvirishvili, ${ }^{155}$ A. Moeller, ${ }^{155}$ J. Nachtman, ${ }^{155}$ H. Ogul, ${ }^{155}$,ttt

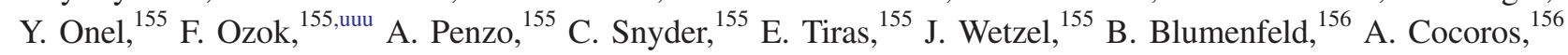
N. Eminizer, ${ }^{156}$ D. Fehling, ${ }^{156}$ L. Feng, ${ }^{156}$ A. V. Gritsan, ${ }^{156}$ W. T. Hung, ${ }^{156}$ P. Maksimovic, ${ }^{156}$ J. Roskes, ${ }^{156}$ U. Sarica, ${ }^{156}$ M. Swartz, ${ }^{156}$ M. Xiao, ${ }^{156}$ A. Al-bataineh ${ }^{157}$ P. Baringer ${ }^{157}$ A. Bean, ${ }^{157}$ S. Boren, ${ }^{157}$ J. Bowen, ${ }^{157}$ A. Bylinkin, ${ }^{157}$ J. Castle, ${ }^{157}$ S. Khalil, ${ }^{157}$ A. Kropivnitskaya, ${ }^{157}$ D. Majumder, ${ }^{157}$ W. Mcbrayer, ${ }^{157}$ M. Murray, ${ }^{157}$ C. Rogan, ${ }^{157}$ S. Sanders, ${ }^{157}$ E. Schmitz, ${ }^{157}$ J. D. Tapia Takaki, ${ }^{157}$ Q. Wang, ${ }^{157}$ S. Duric, ${ }^{158}$ A. Ivanov, ${ }^{158}$ K. Kaadze, ${ }^{158}$ D. Kim, ${ }^{158}$ Y. Maravin, ${ }^{158}$ D. R. Mendis, ${ }^{158}$ T. Mitchell, ${ }^{158}$ A. Modak, ${ }^{158}$ A. Mohammadi, ${ }^{158}$ F. Rebassoo, ${ }^{159}$ D. Wright, ${ }^{159}$ A. Baden, ${ }^{160}$ O. Baron, ${ }^{160}$ A. Belloni, ${ }^{160}$ S. C. Eno, ${ }^{160}$ Y. Feng, ${ }^{160}$ C. Ferraioli, ${ }^{160}$ N. J. Hadley, ${ }^{160}$ S. Jabeen, ${ }^{160}$ G. Y. Jeng, ${ }^{160}$ R. G. Kellogg,${ }^{160}$ J. Kunkle, ${ }^{160}$ A. C. Mignerey, ${ }^{160}$ S. Nabili, ${ }^{160}$ F. Ricci-Tam, ${ }^{160}$ M. Seidel, ${ }^{160}$ Y. H. Shin, ${ }^{160}$ A. Skuja, ${ }^{160}$ S. C. Tonwar, ${ }^{160}$ K. Wong, ${ }^{160}$ D. Abercrombie, ${ }^{161}$ B. Allen, ${ }^{161}$ V. Azzolini, ${ }^{161}$ A. Baty ${ }^{161}$ R. Bi, ${ }^{161}$ S. Brandt,${ }^{161}$ W. Busza, ${ }^{161}$ I. A. Cali, ${ }^{161}$ M. D’Alfonso, ${ }^{161}$ G. Gomez Ceballos, ${ }^{161}$ M. Goncharov, ${ }^{161}$ P. Harris, ${ }^{161}$ D. Hsu, ${ }^{161}$ M. Hu, ${ }^{161}$ Y. Iiyama, ${ }^{161}$ M. Klute, ${ }^{161}$ D. Kovalskyi, ${ }^{161}$ Y.-J. Lee, ${ }^{161}$ P. D. Luckey, ${ }^{161}$ B. Maier, ${ }^{161}$ A. C. Marini ${ }^{161}$ C. Mcginn, ${ }^{161}$ C. Mironov, ${ }^{161}$ S. Narayanan, ${ }^{161}$ X. Niu, ${ }^{161}$ C. Paus, ${ }^{161}$ D. Rankin, ${ }^{161}$ C. Roland, ${ }^{161}$ G. Roland, ${ }^{161}$ Z. Shi, ${ }^{161}$ G. S. F. Stephans, ${ }^{161}$ K. Sumorok, ${ }^{161}$ K. Tatar, ${ }^{161}$

D. Velicanu, ${ }^{161}$ J. Wang, ${ }^{161}$ T. W. Wang, ${ }^{161}$ B. Wyslouch, ${ }^{161}$ A. C. Benvenuti, ${ }^{162, a}$ R. M. Chatterjee, ${ }^{162}$ A. Evans, ${ }^{162}$ P. Hansen, ${ }^{162}$ J. Hiltbrand, ${ }^{162}$ Sh. Jain, ${ }^{162}$ S. Kalafut, ${ }^{162}$ M. Krohn, ${ }^{162}$ Y. Kubota, ${ }^{162}$ Z. Lesko, ${ }^{162}$ J. Mans, ${ }^{162}$ R. Rusack, ${ }^{162}$ M. A. Wadud, ${ }^{162}$ J. G. Acosta, ${ }^{163}$ S. Oliveros, ${ }^{163}$ E. Avdeeva, ${ }^{164}$ K. Bloom, ${ }^{164}$ D. R. Claes, ${ }^{164}$ C. Fangmeier, ${ }^{164}$ F. Golf, ${ }^{164}$ R. Gonzalez Suarez, ${ }^{164}$ R. Kamalieddin, ${ }^{164}$ I. Kravchenko, ${ }^{164}$ J. Monroy, ${ }^{164}$ J. E. Siado, ${ }^{164}$ G. R. Snow, ${ }^{164}$ B. Stieger, ${ }^{164}$

A. Godshalk, ${ }^{165}$ C. Harrington, ${ }^{165}$ I. Iashvili, ${ }^{165}$ A. Kharchilava, ${ }^{165}$ C. Mclean, ${ }^{165}$ D. Nguyen, ${ }^{165}$ A. Parker, ${ }^{165}$

S. Rappoccio, ${ }^{165}$ B. Roozbahani, ${ }^{165}$ G. Alverson, ${ }^{166}$ E. Barberis, ${ }^{166}$ C. Freer, ${ }^{166}$ Y. Haddad, ${ }^{166}$ A. Hortiangtham, ${ }^{166}$ G. Madigan, ${ }^{166}$ D. M. Morse, ${ }^{166}$ T. Orimoto, ${ }^{166}$ A. Tishelman-charny, ${ }^{166}$ T. Wamorkar, ${ }^{166}$ B. Wang, ${ }^{166}$ A. Wisecarver, ${ }^{166}$ D. Wood, ${ }^{166}$ S. Bhattacharya, ${ }^{167}$ J. Bueghly, ${ }^{167}$ O. Charaf, ${ }^{167}$ T. Gunter,${ }^{167}$ K. A. Hahn, ${ }^{167}$ N. Odell, ${ }^{167}$ M. H. Schmitt, ${ }^{167}$ K. Sung, ${ }^{167}$ M. Trovato, ${ }^{167}$ M. Velasco,${ }^{167}$ R. Bucci, ${ }^{168}$ N. Dev, ${ }^{168}$ R. Goldouzian, ${ }^{168}$ M. Hildreth, ${ }^{168}$ K. Hurtado Anampa, ${ }^{168}$ C. Jessop, ${ }^{168}$ D. J. Karmgard, ${ }^{168}$ K. Lannon, ${ }^{168}$ W. Li, ${ }^{168}$ N. Loukas, ${ }^{168}$ N. Marinelli, ${ }^{168}$ F. Meng, ${ }^{168}$ C. Mueller, ${ }^{168}$ Y. Musienko, ${ }^{168, \mathrm{~mm}}$ M. Planer, ${ }^{168}$ R. Ruchti, ${ }^{168}$ P. Siddireddy, ${ }^{168}$ G. Smith, ${ }^{168}$ S. Taroni, ${ }^{168}$ M. Wayne, ${ }^{168}$ A. Wightman, ${ }^{168}$ M. Wolf, ${ }^{168}$ A. Woodard, ${ }^{168}$ J. Alimena, ${ }^{169}$ L. Antonelli, ${ }^{169}$ B. Bylsma, ${ }^{169}$ L. S. Durkin, ${ }^{169}$ S. Flowers, ${ }^{169}$ B. Francis, ${ }^{169}$ C. Hill, ${ }^{169}$ W. Ji, ${ }^{169}$ T. Y. Ling, ${ }^{169}$ W. Luo, ${ }^{169}$ B. L. Winer, ${ }^{169}$ S. Cooperstein, ${ }^{170}$ G. Dezoort, ${ }^{170}$ P. Elmer, ${ }^{170}$ J. Hardenbrook, ${ }^{170}$ N. Haubrich, ${ }^{170}$ S. Higginbotham, ${ }^{170}$ A. Kalogeropoulos, ${ }^{170}$ S. Kwan, ${ }^{170}$ D. Lange, ${ }^{170}$ M. T. Lucchini, ${ }^{170}$ J. Luo, ${ }^{170}$ D. Marlow, ${ }^{170}$ K. Mei, ${ }^{170}$ I. Ojalvo, ${ }^{170}$ J. Olsen, ${ }^{170}$ C. Palmer, ${ }^{170}$ P. Piroué, ${ }^{170}$ J. Salfeld-Nebgen, ${ }^{170}$ D. Stickland, ${ }^{170}$ C. Tully, ${ }^{170}$ S. Malik, ${ }^{171}$ S. Norberg, ${ }^{171}$ A. Barker, ${ }^{172}$ V. E. Barnes, ${ }^{172}$ S. Das, ${ }^{172}$ L. Gutay, ${ }^{172}$ M. Jones, ${ }^{172}$ A. W. Jung, ${ }^{172}$ A. Khatiwada, ${ }^{172}$ B. Mahakud, ${ }^{172}$ D. H. Miller, ${ }^{172}$ N. Neumeister, ${ }^{172}$ C. C. Peng, ${ }^{172}$ S. Piperov, ${ }^{172}$ H. Qiu, ${ }^{172}$ J. F. Schulte, ${ }^{172}$ J. Sun, ${ }^{172}$ F. Wang, ${ }^{172}$ R. Xiao, ${ }^{172}$ W. Xie, ${ }^{172}$ T. Cheng, ${ }^{173}$ J. Dolen, ${ }^{173}$ N. Parashar, ${ }^{173}$ Z. Chen, ${ }^{174}$ K. M. Ecklund, ${ }^{174}$ S. Freed, ${ }^{174}$ F. J. M. Geurts, ${ }^{174}$ M. Kilpatrick, ${ }^{174}$ Arun Kumar, ${ }^{174}$ W. Li, ${ }^{174}$ B. P. Padley, ${ }^{174}$ R. Redjimi, ${ }^{174}$ J. Roberts, ${ }^{174}$ J. Rorie, ${ }^{174}$ W. Shi, ${ }^{174}$ Z. Tu, ${ }^{174}$ A. Zhang, ${ }^{174}$ A. Bodek, ${ }^{175}$ P. de Barbaro, ${ }^{175}$ R. Demina, ${ }^{175}$ Y. t. Duh, ${ }^{175}$ J. L. Dulemba, ${ }^{175}$ C. Fallon, ${ }^{175}$ T. Ferbel, ${ }^{175}$ M. Galanti, ${ }^{175}$ A. Garcia-Bellido, ${ }^{175}$ J. Han, ${ }^{175}$ O. Hindrichs, ${ }^{175}$ A. Khukhunaishvili, ${ }^{175}$ 
E. Ranken, ${ }^{175}$ P. Tan, ${ }^{175}$ R. Taus, ${ }^{175}$ B. Chiarito, ${ }^{176}$ J. P. Chou, ${ }^{176}$ Y. Gershtein, ${ }^{176}$ E. Halkiadakis, ${ }^{176}$ A. Hart, ${ }^{176}$ M. Heindl, ${ }^{176}$ E. Hughes, ${ }^{176}$ S. Kaplan, ${ }^{176}$ R. Kunnawalkam Elayavalli, ${ }^{176}$ S. Kyriacou, ${ }^{176}$ I. Laflotte, ${ }^{176}$ A. Lath, ${ }^{176}$ R. Montalvo, ${ }^{176}$

K. Nash, ${ }^{176}$ M. Osherson, ${ }^{176}$ H. Saka, ${ }^{176}$ S. Salur, ${ }^{176}$ S. Schnetzer, ${ }^{176}$ D. Sheffield, ${ }^{176}$ S. Somalwar, ${ }^{176}$ R. Stone, ${ }^{176}$ S. Thomas, ${ }^{176}$ P. Thomassen, ${ }^{176}$ H. Acharya, ${ }^{177}$ A. G. Delannoy, ${ }^{177}$ J. Heideman, ${ }^{177}$ G. Riley, ${ }^{177}$ S. Spanier, ${ }^{177}$ O. Bouhali, ${ }^{178, v v v}$ A. Celik, ${ }^{178}$ M. Dalchenko, ${ }^{178}$ M. De Mattia, ${ }^{178}$ A. Delgado, ${ }^{178}$ S. Dildick, ${ }^{178}$ R. Eusebi, ${ }^{178}$ J. Gilmore ${ }^{178}$ T. Huang, ${ }^{178}$ T. Kamon, ${ }^{178, w w w}$ S. Luo, ${ }^{178}$ D. Marley, ${ }^{178}$ R. Mueller, ${ }^{178}$ D. Overton, ${ }^{178}$ L. Perniè, ${ }^{178}$ D. Rathjens, ${ }^{178}$ A. Safonov, ${ }^{178}$ N. Akchurin, ${ }^{179}$ J. Damgov, ${ }^{179}$ F. De Guio, ${ }^{179}$ P. R. Dudero, ${ }^{179}$ S. Kunori, ${ }^{179}$ K. Lamichhane ${ }^{179}$ S. W. Lee, ${ }^{179}$ T. Mengke, ${ }^{179}$ S. Muthumuni, ${ }^{179}$ T. Peltola, ${ }^{179}$ S. Undleeb, ${ }^{179}$ I. Volobouev, ${ }^{179}$ Z. Wang, ${ }^{179}$ A. Whitbeck, ${ }^{179}$ S. Greene, ${ }^{180}$ A. Gurrola, ${ }^{180}$ R. Janjam, ${ }^{180}$ W. Johns, ${ }^{180}$ C. Maguire, ${ }^{180}$ A. Melo, ${ }^{180}$ H. Ni, ${ }^{180}$ K. Padeken, ${ }^{180}$ F. Romeo, ${ }^{180}$ P. Sheldon, ${ }^{180}$ S. Tuo, ${ }^{180}$ J. Velkovska, ${ }^{180}$ M. Verweij, ${ }^{180}$ Q. Xu, ${ }^{180}$ M. W. Arenton, ${ }^{181}$ P. Barria, ${ }^{181}$ B. Cox, ${ }^{181}$ R. Hirosky, ${ }^{181}$ M. Joyce, ${ }^{181}$ A. Ledovskoy, ${ }^{181}$ H. Li, ${ }^{181}$ C. Neu, ${ }^{181}$ T. Sinthuprasith, ${ }^{181}$ Y. Wang, ${ }^{181}$ E. Wolfe, ${ }^{181}$ F. Xia, ${ }^{181}$ R. Harr, ${ }^{182}$ P. E. Karchin, ${ }^{182}$ N. Poudyal, ${ }^{182}$ J. Sturdy, ${ }^{182}$ P. Thapa, ${ }^{182}$ S. Zaleski, ${ }^{182}$ J. Buchanan, ${ }^{183}$ C. Caillol, ${ }^{183}$ D. Carlsmith, ${ }^{183}$ S. Dasu, ${ }^{183}$ I. De Bruyn, ${ }^{183}$ L. Dodd, ${ }^{183}$ B. Gomber, ${ }^{183, x x x}$ M. Grothe, ${ }^{183}$ M. Herndon, ${ }^{183}$ A. Hervé, ${ }^{183}$ U. Hussain, ${ }^{183}$ P. Klabbers, ${ }^{183}$ A. Lanaro, ${ }^{183}$ K. Long, ${ }^{183}$ R. Loveless, ${ }^{183}$ T. Ruggles, ${ }^{183}$ A. Savin, ${ }^{183}$ V. Sharma, ${ }^{183}$ N. Smith, ${ }^{183}$ W. H. Smith, ${ }^{183}$ and N. Woods ${ }^{183}$

\title{
(CMS Collaboration)
}

\author{
${ }^{1}$ Yerevan Physics Institute, Yerevan, Armenia \\ ${ }^{2}$ Institut für Hochenergiephysik, Wien, Austria \\ ${ }^{3}$ Institute for Nuclear Problems, Minsk, Belarus \\ ${ }^{4}$ Universiteit Antwerpen, Antwerpen, Belgium \\ ${ }^{5}$ Vrije Universiteit Brussel, Brussel, Belgium \\ ${ }^{6}$ Université Libre de Bruxelles, Bruxelles, Belgium \\ ${ }^{7}$ Ghent University, Ghent, Belgium \\ ${ }^{8}$ Université Catholique de Louvain, Louvain-la-Neuve, Belgium \\ ${ }^{9}$ Centro Brasileiro de Pesquisas Fisicas, Rio de Janeiro, Brazil \\ ${ }^{10}$ Universidade do Estado do Rio de Janeiro, Rio de Janeiro, Brazil \\ ${ }^{11 a}$ Universidade Estadual Paulista, São Paulo, Brazil \\ ${ }^{11 \mathrm{~b}}$ Universidade Federal do ABC, São Paulo, Brazil \\ ${ }^{12}$ Institute for Nuclear Research and Nuclear Energy, Bulgarian Academy of Sciences, Sofia, Bulgaria \\ ${ }^{13}$ University of Sofia, Sofia, Bulgaria \\ ${ }^{14}$ Beihang University, Beijing, China \\ ${ }^{15}$ Institute of High Energy Physics, Beijing, China \\ ${ }^{16}$ State Key Laboratory of Nuclear Physics and Technology, Peking University, Beijing, China \\ ${ }^{17}$ Tsinghua University, Beijing, China \\ ${ }^{18}$ Universidad de Los Andes, Bogota, Colombia \\ ${ }^{19}$ University of Split, Faculty of Electrical Engineering, Mechanical Engineering and Naval Architecture, \\ Split, Croatia \\ ${ }^{20}$ University of Split, Faculty of Science, Split, Croatia \\ ${ }^{21}$ Institute Rudjer Boskovic, Zagreb, Croatia \\ ${ }^{22}$ University of Cyprus, Nicosia, Cyprus \\ ${ }^{23}$ Charles University, Prague, Czech Republic \\ ${ }^{24}$ Escuela Politecnica Nacional, Quito, Ecuador \\ ${ }^{25}$ Universidad San Francisco de Quito, Quito, Ecuador \\ ${ }^{26}$ Academy of Scientific Research and Technology of the Arab Republic of Egypt, \\ Egyptian Network of High Energy Physics, Cairo, Egypt \\ ${ }^{27}$ National Institute of Chemical Physics and Biophysics, Tallinn, Estonia \\ ${ }^{28}$ Department of Physics, University of Helsinki, Helsinki, Finland \\ ${ }^{29}$ Helsinki Institute of Physics, Helsinki, Finland \\ ${ }^{30}$ Lappeenranta University of Technology, Lappeenranta, Finland \\ ${ }^{31}$ IRFU, CEA, Université Paris-Saclay, Gif-sur-Yvette, France \\ ${ }^{32}$ Laboratoire Leprince-Ringuet, Ecole polytechnique, \\ CNRS/IN2P3, Université Paris-Saclay, Palaiseau, France \\ ${ }^{33}$ Université de Strasbourg, CNRS, IPHC UMR 7178, Strasbourg, France
}


${ }^{34}$ Centre de Calcul de l'Institut National de Physique Nucleaire et de Physique des Particules, CNRS/IN2P3, Villeurbanne, France

${ }^{35}$ Université de Lyon, Université Claude Bernard Lyon 1, CNRS-IN2P3, Institut de Physique Nucléaire de Lyon, Villeurbanne, France

${ }^{36}$ Georgian Technical University, Tbilisi, Georgia

${ }^{37}$ Tbilisi State University, Tbilisi, Georgia

${ }^{38}$ RWTH Aachen University, I. Physikalisches Institut, Aachen, Germany

${ }^{39}$ RWTH Aachen University, III. Physikalisches Institut A, Aachen, Germany

${ }^{40}$ RWTH Aachen University, III. Physikalisches Institut B, Aachen, Germany

${ }^{41}$ Deutsches Elektronen-Synchrotron, Hamburg, Germany

${ }^{42}$ University of Hamburg, Hamburg, Germany

${ }^{43}$ Karlsruher Institut fuer Technologie, Karlsruhe, Germany

${ }^{44}$ Institute of Nuclear and Particle Physics (INPP), NCSR Demokritos, Aghia Paraskevi, Greece

${ }^{45}$ National and Kapodistrian University of Athens, Athens, Greece

${ }^{46}$ National Technical University of Athens, Athens, Greece

${ }^{47}$ University of Ioánnina, Ioánnina, Greece

${ }^{48}$ MTA-ELTE Lendület CMS Particle and Nuclear Physics Group, Eötvös Loránd University, Budapest, Hungary

${ }^{49}$ Wigner Research Centre for Physics, Budapest, Hungary

${ }^{50}$ Institute of Nuclear Research ATOMKI, Debrecen, Hungary

${ }^{51}$ Institute of Physics, University of Debrecen, Debrecen, Hungary

${ }^{52}$ Indian Institute of Science (IISc), Bangalore, India

${ }^{53}$ National Institute of Science Education and Research, HBNI, Bhubaneswar, India

${ }^{54}$ Panjab University, Chandigarh, India

${ }^{55}$ University of Delhi, Delhi, India

${ }^{56}$ Saha Institute of Nuclear Physics, HBNI, Kolkata,India

${ }^{57}$ Indian Institute of Technology Madras, Madras, India

${ }^{58}$ Bhabha Atomic Research Centre, Mumbai, India

${ }^{59}$ Tata Institute of Fundamental Research-A, Mumbai, India

${ }^{60}$ Tata Institute of Fundamental Research-B, Mumbai, India

${ }^{61}$ Indian Institute of Science Education and Research (IISER), Pune, India

${ }^{62}$ Institute for Research in Fundamental Sciences (IPM), Tehran, Iran

${ }^{63}$ University College Dublin, Dublin, Ireland

${ }^{64 \mathrm{a}}$ INFN Sezione di Bari, Bari, Italy

${ }^{64 \mathrm{~b}}$ Università di Bari, Bari, Italy

${ }^{64 \mathrm{c}}$ Politecnico di Bari, Bari, Italy

${ }^{65 \mathrm{a}}$ INFN Sezione di Bologna, Bologna, Italy

${ }^{65 \mathrm{~b}}$ Università di Bologna, Bologna, Italy

${ }^{66 a}$ INFN Sezione di Catania, Catania, Italy

${ }^{66 \mathrm{~b}}$ Università di Catania, Catania, Italy

${ }^{67 \mathrm{a}}$ INFN Sezione di Firenze, Firenze, Italy

${ }^{67 \mathrm{~b}}$ Università di Firenze, Firenze, Italy

${ }^{68}$ INFN Laboratori Nazionali di Frascati, Frascati, Italy

${ }^{69}$ INFN Sezione di Genova, Genova, Italy

${ }^{69 \mathrm{~b}}$ Università di Genova, Genova, Italy

${ }^{70 a}$ INFN Sezione di Milano-Bicocca, Milano, Italy

${ }^{70 \mathrm{~b}}$ Università di Milano-Bicocca, Milano, Italy

${ }^{71 a}$ INFN Sezione di Napoli', Napoli, Italy

${ }^{71 \mathrm{~b}}$ Università di Napoli 'Federico II', Napoli, Italy

${ }^{71 \mathrm{c}}$ Università della Basilicata, Potenza, Italy

${ }^{71 d}$ Università G. Marconi, Roma, Italy

${ }^{72 a}$ INFN Sezione di Padova, Padova, Italy

${ }^{72 \mathrm{~b}}$ Università di Padova, Padova, Italy

${ }^{72 \mathrm{c}}$ Università di Trento, Trento, Italy

${ }^{73}$ INFN Sezione di Pavia, Pavia, Italy

${ }^{73 b}$ Università di Pavia, Pavia, Italy

${ }^{74 a}$ INFN Sezione di Perugia, Perugia, Italy

${ }^{74 \mathrm{~b}}$ Università di Perugia, Perugia, Italy

${ }^{75 a}$ INFN Sezione di Pisa, Pisa, Italy

${ }^{75 b}$ Università di Pisa, Pisa, Italy 
${ }^{75 \mathrm{c}}$ Scuola Normale Superiore di Pisa, Pisa, Italy

${ }^{76 a}$ INFN Sezione di Roma, Rome, Italy

${ }^{76 \mathrm{~b}}$ Sapienza Università di Roma, Rome, Italy

${ }^{77 a}$ INFN Sezione di Torino, Torino, Italy

${ }^{77 b}$ Università di Torino, Torino, Italy

${ }^{77 \mathrm{c}}$ Università del Piemonte Orientale, Novara, Italy

${ }^{78 \mathrm{a}}$ INFN Sezione di Trieste, Trieste, Italy

${ }^{78 b}$ Università di Trieste, Trieste, Italy

${ }^{79}$ Kyungpook National University, Daegu, Korea

${ }^{80}$ Chonnam National University, Institute for Universe and Elementary Particles, Kwangju, Korea

${ }^{81}$ Hanyang University, Seoul, Korea

${ }^{82}$ Korea University, Seoul, Korea

${ }^{83}$ Sejong University, Seoul, Korea

${ }^{84}$ Seoul National University, Seoul, Korea

${ }^{85}$ University of Seoul, Seoul, Korea

${ }^{86}$ Sungkyunkwan University, Suwon, Korea

${ }^{87}$ Riga Technical University, Riga, Latvia

${ }^{88}$ Vilnius University, Vilnius, Lithuania

${ }^{89}$ National Centre for Particle Physics, Universiti Malaya, Kuala Lumpur, Malaysia

${ }^{90}$ Universidad de Sonora (UNISON), Hermosillo, Mexico

${ }^{91}$ Centro de Investigacion y de Estudios Avanzados del IPN, Mexico City, Mexico

${ }^{92}$ Universidad Iberoamericana, Mexico City, Mexico

${ }^{93}$ Benemerita Universidad Autonoma de Puebla, Puebla, Mexico

${ }^{94}$ Universidad Autónoma de San Luis Potosí, San Luis Potosí, Mexico

${ }^{95}$ University of Auckland, Auckland, New Zealand

${ }^{96}$ University of Canterbury, Christchurch, New Zealand

${ }^{97}$ National Centre for Physics, Quaid-I-Azam University, Islamabad, Pakistan

${ }^{98}$ National Centre for Nuclear Research, Swierk, Poland

${ }^{99}$ Institute of Experimental Physics, Faculty of Physics, University of Warsaw, Warsaw, Poland

${ }^{100}$ Laboratório de Instrumentação e Física Experimental de Partículas, Lisboa, Portugal

${ }^{101}$ Joint Institute for Nuclear Research, Dubna, Russia

${ }^{102}$ Petersburg Nuclear Physics Institute, Gatchina (St. Petersburg), Russia

${ }^{103}$ Institute for Nuclear Research, Moscow, Russia

${ }^{104}$ Institute for Theoretical and Experimental Physics, Moscow, Russia

${ }^{105}$ Moscow Institute of Physics and Technology, Moscow, Russia

${ }^{106}$ National Research Nuclear University 'Moscow Engineering Physics Institute' (MEPhI),

Moscow, Russia

${ }^{107}$ P.N. Lebedev Physical Institute, Moscow, Russia

${ }^{108}$ Skobeltsyn Institute of Nuclear Physics, Lomonosov Moscow State University, Moscow, Russia

${ }^{109}$ Novosibirsk State University (NSU), Novosibirsk, Russia

${ }^{110}$ Institute for High Energy Physics of National Research Centre 'Kurchatov Institute', Protvino, Russia

${ }^{111}$ National Research Tomsk Polytechnic University, Tomsk, Russia

${ }^{112}$ University of Belgrade, Faculty of Physics and Vinca Institute of Nuclear Sciences, Belgrade, Serbia

${ }^{113}$ Centro de Investigaciones Energéticas Medioambientales y Tecnológicas (CIEMAT), Madrid, Spain

${ }^{114}$ Universidad Autónoma de Madrid, Madrid, Spain

${ }^{115}$ Universidad de Oviedo, Oviedo, Spain

${ }^{116}$ Instituto de Física de Cantabria (IFCA), CSIC-Universidad de Cantabria, Santander, Spain

${ }^{117}$ University of Ruhuna, Department of Physics, Matara, Sri Lanka

${ }^{118}$ CERN, European Organization for Nuclear Research, Geneva, Switzerland

${ }^{119}$ Paul Scherrer Institut, Villigen, Switzerland

${ }^{120}$ ETH Zurich - Institute for Particle Physics and Astrophysics (IPA), Zurich, Switzerland

${ }^{121}$ Universität Zürich, Zurich, Switzerland

${ }^{122}$ National Central University, Chung-Li, Taiwan

${ }^{123}$ National Taiwan University (NTU), Taipei, Taiwan

${ }^{124}$ Chulalongkorn University, Faculty of Science, Department of Physics, Bangkok, Thailand

${ }^{125}$ Çukurova University, Physics Department, Science and Art Faculty, Adana, Turkey

${ }^{126}$ Middle East Technical University, Physics Department, Ankara, Turkey

${ }^{127}$ Bogazici University, Istanbul, Turkey

${ }^{128}$ Istanbul Technical University, Istanbul, Turkey

${ }^{129}$ Institute for Scintillation Materials of National Academy of Science of Ukraine, Kharkov, Ukraine 


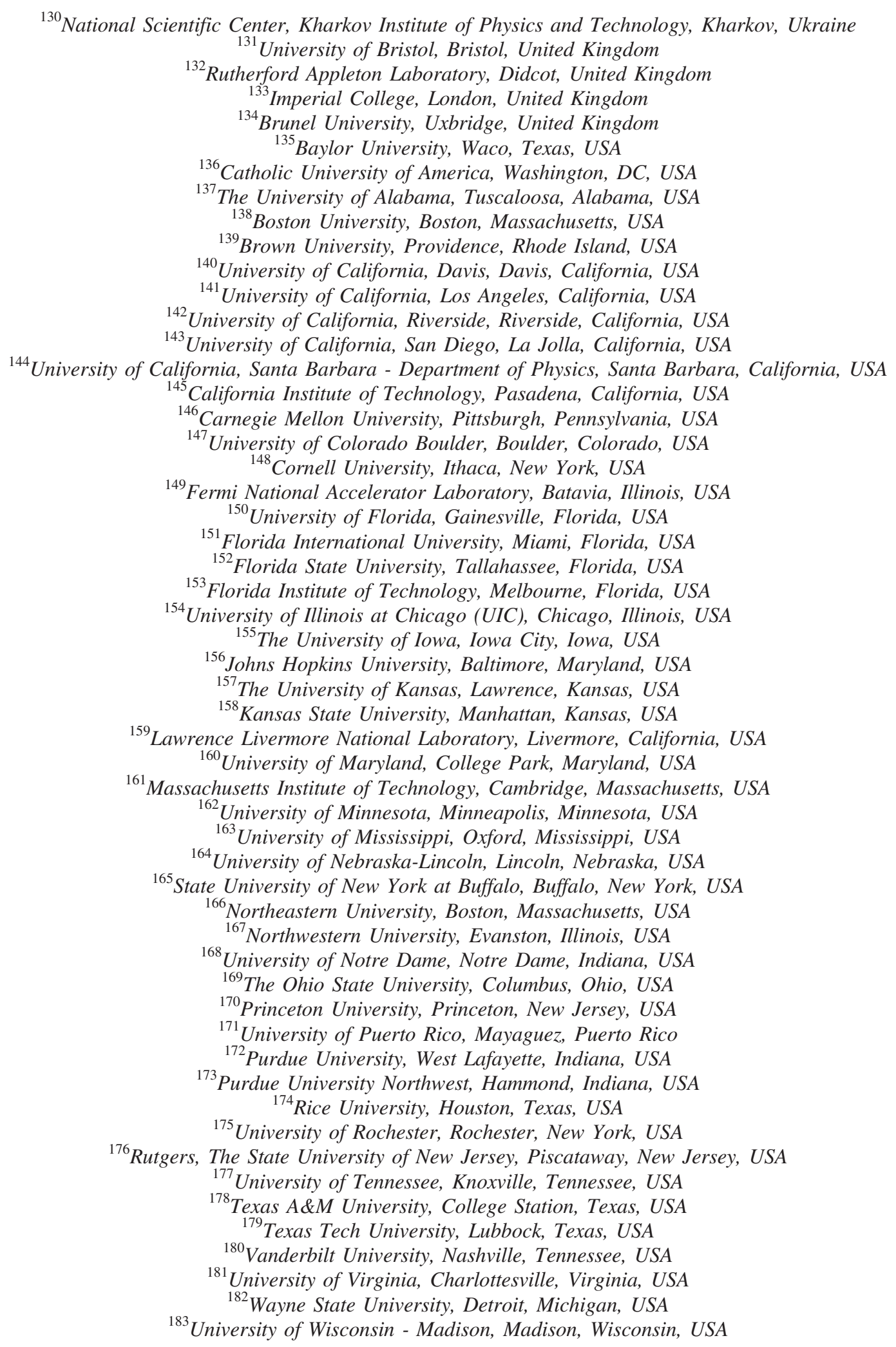

\footnotetext{
${ }^{\mathrm{a}}$ Deceased.

${ }^{\mathrm{b}}$ Also at Vienna University of Technology, Vienna, Austria.

${ }^{\mathrm{c}}$ Also at IRFU, CEA, Université Paris-Saclay, Gif-sur-Yvette, France.

${ }^{\mathrm{d}}$ Also at Universidade Estadual de Campinas, Campinas, Brazil.

${ }^{\mathrm{e}}$ Also at Federal University of Rio Grande do Sul, Porto Alegre, Brazil.
} 
${ }^{\mathrm{f}}$ Also at Université Libre de Bruxelles, Bruxelles, Belgium.

${ }^{g}$ Also at University of Chinese Academy of Sciences, Beijing, China.

${ }^{\mathrm{h}}$ Also at Institute for Theoretical and Experimental Physics, Moscow, Russia.

${ }^{\mathrm{i}}$ Also at Joint Institute for Nuclear Research, Dubna, Russia.

${ }^{j}$ Also at Helwan University, Cairo, Egypt.

${ }^{\mathrm{k}}$ Also at Zewail City of Science and Technology, Zewail, Egypt.

${ }^{1}$ Also at British University in Egypt, Cairo, Egypt.

${ }^{\mathrm{m}}$ Also at Suez University, Suez, Egypt.

${ }^{\mathrm{n}}$ Also at Department of Physics, King Abdulaziz University, Jeddah, Saudi Arabia.

${ }^{\circ}$ Also at Université de Haute Alsace, Mulhouse, France.

${ }^{\mathrm{p}}$ Also at Skobeltsyn Institute of Nuclear Physics, Lomonosov Moscow State University, Moscow, Russia.

${ }^{\mathrm{q}}$ Also at CERN, European Organization for Nuclear Research, Geneva, Switzerland.

${ }^{\mathrm{r}}$ Also at RWTH Aachen University, III. Physikalisches Institut A, Aachen, Germany.

${ }^{\mathrm{s}}$ Also at University of Hamburg, Hamburg, Germany.

tAlso at Brandenburg University of Technology, Cottbus, Germany.

"Also at Institute of Physics, University of Debrecen, Debrecen, Hungary.

${ }^{v}$ Also at Institute of Nuclear Research ATOMKI, Debrecen, Hungary.

${ }^{w}$ Also at MTA-ELTE Lendület CMS Particle and Nuclear Physics Group, Eötvös Loránd University, Budapest, Hungary.

${ }^{\mathrm{x}}$ Also at IIT Bhubaneswar, Bhubaneswar, India.

${ }^{\mathrm{y}}$ Also at Institute of Physics, Bhubaneswar, India.

${ }^{z}$ Also at Shoolini University, Solan, India.

${ }^{\text {aa }}$ Also at University of Visva-Bharati, Santiniketan, India.

${ }^{\text {bb }}$ Also at Isfahan University of Technology, Isfahan, Iran.

${ }^{c c}$ Also at Plasma Physics Research Center, Science and Research Branch, Islamic Azad University, Tehran, Iran.

${ }^{\mathrm{dd}}$ Also at Italian National Agency for New Technologies, Energy and Sustainable Economic Development, Bologna, Italy.

${ }^{e e}$ Also at Università degli Studi di Siena, Siena, Italy.

${ }^{\mathrm{ff}}$ Also at Scuola Normale e Sezione dell'INFN, Pisa, Italy.

${ }^{\text {gg }}$ Also at Kyunghee University, Seoul, Korea.

${ }^{\text {hh }}$ Also at Riga Technical University, Riga, Latvia.

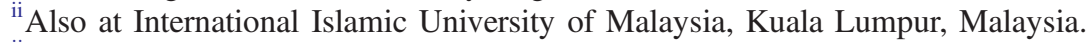

${ }^{\mathrm{jj}}$ Also at Malaysian Nuclear Agency, MOSTI, Kajang, Malaysia.

${ }^{\mathrm{kk}}$ Also at Consejo Nacional de Ciencia y Tecnología, Mexico City, Mexico.

${ }^{11}$ Also at Warsaw University of Technology, Institute of Electronic Systems, Warsaw, Poland.

${ }^{\mathrm{mm}}$ Also at Institute for Nuclear Research, Moscow, Russia.

${ }^{n n}$ Also at National Research Nuclear University 'Moscow Engineering Physics Institute' (MEPhI), Moscow, Russia.

${ }^{\circ 0}$ Also at St. Petersburg State Polytechnical University, St. Petersburg, Russia.

${ }^{\mathrm{pp}}$ Also at University of Florida, Gainesville, Florida, USA.

${ }^{\mathrm{qq}}$ Also at P.N. Lebedev Physical Institute, Moscow, Russia.

${ }^{\mathrm{rr}}$ Also at California Institute of Technology, Pasadena, California, USA.

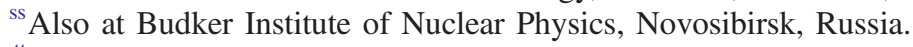

"Also at Faculty of Physics, University of Belgrade, Belgrade, Serbia.

${ }^{\text {uu }}$ Also at University of Belgrade, Faculty of Physics and Vinca Institute of Nuclear Sciences, Belgrade, Serbia.

${ }^{v v}$ Also at INFN Sezione di Pavia, Università di Pavia, Pavia, Italy.

${ }^{w w}$ Also at National and Kapodistrian University of Athens, Athens, Greece.

${ }^{\mathrm{xx}}$ Also at Universität Zürich, Zurich, Switzerland.

${ }^{y y}$ Also at Stefan Meyer Institute for Subatomic Physics, Vienna, Austria.

${ }^{\mathrm{zz}}$ Also at Adiyaman University, Adiyaman, Turkey.

${ }^{\text {aaa }}$ Also at Istanbul Aydin University, Istanbul, Turkey.

${ }^{\mathrm{bbb}}$ Also at Mersin University, Mersin, Turkey.

${ }^{c c c}$ Also at Piri Reis University, Istanbul, Turkey.

${ }^{\text {ddd }}$ Also at Gaziosmanpasa University, Tokat, Turkey.

${ }^{\text {eee }}$ Also at Ozyegin University, Istanbul, Turkey.

${ }^{\text {fff }}$ Also at Izmir Institute of Technology, Izmir, Turkey.

${ }^{\text {ggg }}$ Also at Marmara University, Istanbul, Turkey.

${ }^{\text {hhh }}$ Also at Kafkas University, Kars, Turkey.

${ }^{i i i}$ Also at Istanbul University, Faculty of Science, Istanbul, Turkey.

${ }^{\mathrm{jij}}$ Also at Istanbul Bilgi University, Istanbul, Turkey.

${ }^{k k k}$ Also at Hacettepe University, Ankara, Turkey.

${ }^{111}$ Also at Rutherford Appleton Laboratory, Didcot, United Kingdom.

${ }^{\mathrm{mmm}}$ Also at School of Physics and Astronomy, University of Southampton, Southampton, United Kingdom. 
${ }^{n n n}$ Also at Monash University, Faculty of Science, Clayton, Australia.

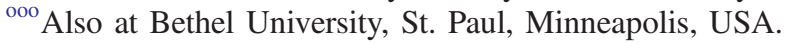

${ }^{\mathrm{ppp}}$ Also at Karamanoğlu Mehmetbey University, Karaman, Turkey.

${ }^{\mathrm{qqq}}$ Also at Purdue University, West Lafayette, Indiana, USA.

${ }^{\mathrm{rrr}}$ Also at Beykent University, Istanbul, Turkey.

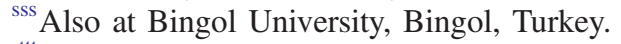

${ }^{\mathrm{ttt}}$ Also at Sinop University, Sinop, Turkey.

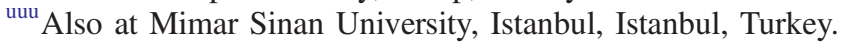

${ }^{\mathrm{vvv}}$ Also at Texas A\&M University at Qatar, Doha, Qatar.

${ }^{w w w}$ Also at Kyungpook National University, Daegu, Korea.

${ }^{\mathrm{xxx}}$ Also at University of Hyderabad, Hyderabad, India. 